Pacific Northwest

National Laboratory

Operated by Battelle for the

U.S. Department of Energy

\title{
Material Balance Assessment for Double-Shell Tank Waste Pipeline Transfer
}

\author{
Y. Onishi \\ B. E. Wells \\ S.A. Hartley \\ C.W. Enderlin \\ M. White
}

September 2002

Prepared for the U.S. Department of Energy under Contract DE-AC06-76RL01830 


\section{DISCLAIMER}

This report was prepared as an account of work sponsored by an agency of the United States Government. Neither the United States Government nor any agency thereof, nor Battelle Memorial Institute nor any of their employees makes any warranty, express or implied, or assumes any legal liability or responsibility for the accuracy, completeness, or usefulness of any information, apparatus, product, or process disclosed or represents that its use would not infringe privately owned rights. Reference herein to any specific commercial product, process, or service by trade name, trademark, manufacturer, or otherwise does not necessarily constitute or imply its endorsement, recommendation, or favoring by the United States Government or any agency thereof, or Battelle Memorial Institute. The views and opinions of authors expressed herein do not necessarily state or reflect those of the United States Government or any agency thereof.

\section{PACIFIC NORTHWEST NATIONAL LABORATORY operated by \\ BATTELLE \\ for the \\ UNITED STATES DEPARTMENT OF ENERGY under Contract DE-AC06-76RL01830}

Printed in the United States of America

$$
\begin{aligned}
& \text { Available to DOE and DOE contractors from the } \\
& \text { Office of Scientific and Technical Information, } \\
& \text { P.O. Box 62, Oak Ridge, TN } 37831-0062 \text {; } \\
& \text { ph: (865) 576-8401 } \\
& \text { fax: (865) 576-5728 } \\
& \text { email: reports @ adonis.osti.gov }
\end{aligned}
$$

Available to the public from the National Technical Information Service, U.S. Department of Commerce, 5285 Port Royal Rd., Springfield, VA 22161

$$
\begin{aligned}
& \text { ph: (800) 553-6847 } \\
& \text { fax: (703) 605-6900 }
\end{aligned}
$$

email: orders@ntis.fedworld.gov

online ordering: http://www.ntis.gov/ordering.htm 
PNNL-13485

Rev. 1

\title{
Material Balance Assessment for Double-Shell Tank Waste Pipeline Transfer
}

\author{
Y. Onishi \\ B.E. Wells \\ S.A. Hartley \\ C.W. Enderlin \\ M. White
}

September 2002

Prepared for

the U.S. Department of Energy

under Contract DE-AC06-76RLO 1830

Pacific Northwest National Laboratory

Richland, WA 99352 


\section{Summary}

Pacific Northwest National Laboratory researchers developed a material balance assessment methodology based on conservation of mass to detect possible waste leaking and mis-routings during the pipeline transfer of double-shell tank waste complicated by the variable waste properties and tank conditions at Hanford. It is intended to be a backup method to pit leak detectors.

The only difference between the original report, PNNL-13485, released in March 2001, and this current report, PNNL-13485 Rev. 1, is that Rev. 1 contains additional information on Micro Motion $^{\mathrm{TM}}$ Coriolis mass flow meters. Otherwise, the two reports are identical.

The main factors causing variable waste properties and tank conditions are waste density changes caused by chemical reactions and gas generation/retention/release, the existence of a crust layer, and waste surface disturbance due to mixer pump operation during the waste transfer. If waste properties and tank conditions were constant, this mass-based material balance methodology could be simplified to a volume-based material balance.

The material balance assessment methodology was applied to three waste transfers: AN-105 first transfer of 911,400 gallons of in-line diluted supernatant liquid; AN-105 second transfer with 673,000 gallons of liquid waste; and AZ-102 slurry transfer of 150,000 gallons. Three instrumentation setups were considered: (A) feed and receiver tank levels and diluent flow meter; (B) flow meter at the beginning of the transfer pipeline and receiver tank level; and (C) diluent, feed, and receiver tank levels.

For constant waste properties and tank conditions, the largest material balance error with optimum instrumentation is 2,200 gallons out of 911,400 gallons transferred (AN-105 first transfer) using instrumentation setup B with Micro Motion Elite ${ }^{\mathrm{TM}}$ CMF 200 or T150 mass flow meter in the transfer pipeline and an Enraf ${ }^{\mathrm{TM}}$ in the receiver tank.

When uncertainties due to variable waste properties and tank conditions were included in the analysis, the material balance errors became much larger, ranging from 13,600 gallons (1.5\% error) with instrumentation setup B to 68,400 gallons (7.2\% error) with instrumentation setup A for the AN-105 first transfer.

An alternative instrumentation setup to reduce these errors would be to have a Micro Motion Elite CMF 200 or T150 mass flow meter at the both ends of the transfer pipeline. Using this alternative setup with variable waste properties and tank conditions, the material balance error from the AN-105 first transfer is reduced from 13,600 gallons to 3,900 gallons in the transfer pipeline. Thus, depending on the operational accuracy needs, one can select

- volume-based material balance equations for constant waste properties and tank conditions

- mass-based material balance equations for variable waste properties and tank conditions with one or combinations of the three instrumentation setups $(\mathrm{A}, \mathrm{B}, \mathrm{C})$ 
- mass-based material balance equations for variable waste properties and tank conditions with the alternative instrumentation setup having mass flow meters at both ends of the transfer pipeline. 


\section{Contents}

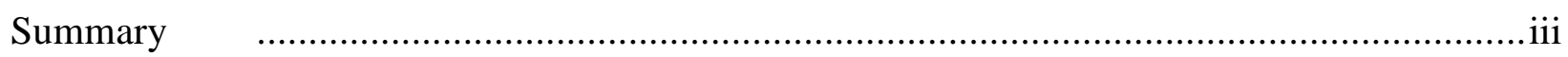

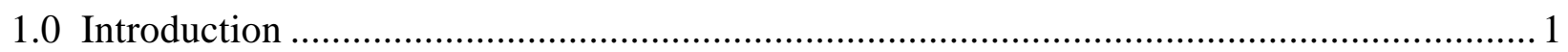

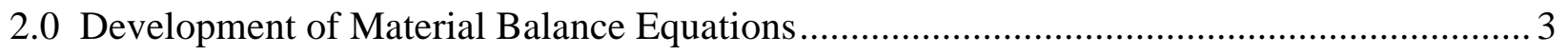

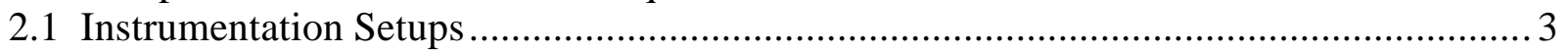

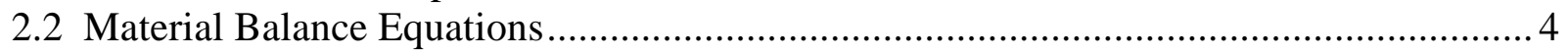

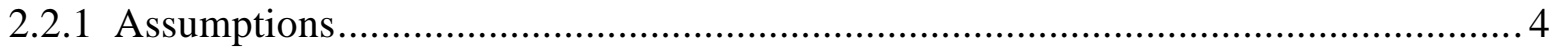

2.2.2 General Material Balance Equations ………........................................................ 4

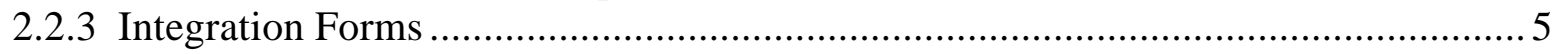

2.3 Simplified Material Balance Equations under Constant Waste

Properties and Tank Conditions........................................................................... 9

3.0 Material Balance Applications …………………....................................................... 11

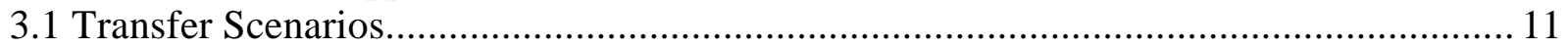

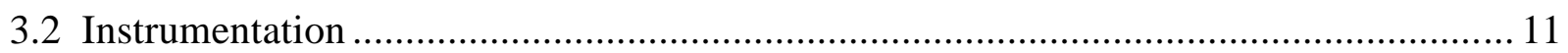

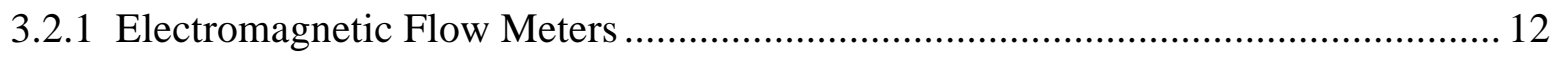

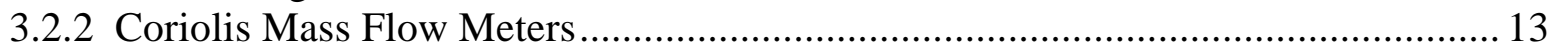

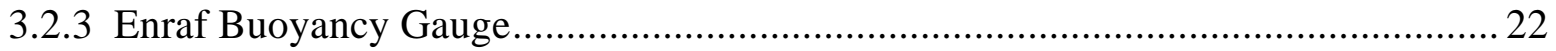

3.3 Statistical Assessment Methodology ……………..................................................... 22

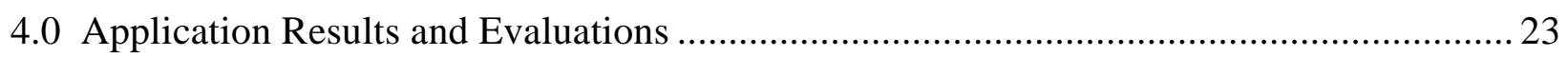

4.1 Constant Waste Properties and Tank Conditions ...................................................... 23

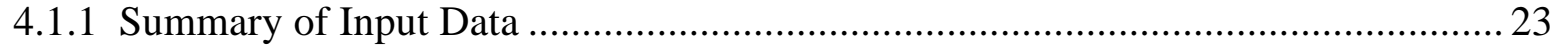

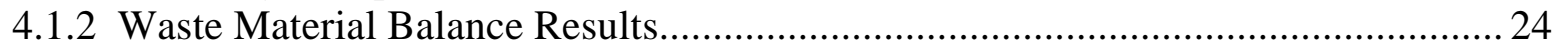

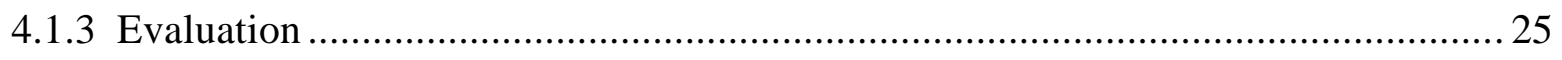

4.2 Variable Waste Properties and Tank Conditions ................................................................ 26

4.2.1 Factors Affecting Accurate Measurements of the Waste Height ................................ 26

4.2.2 Determination of Height Measurement Accuracy .................................................... 29

4.2.3 Factors Affecting Accurate Measurements of the Waste Flow Rate ......................... 29

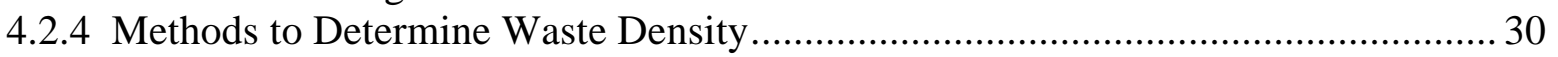

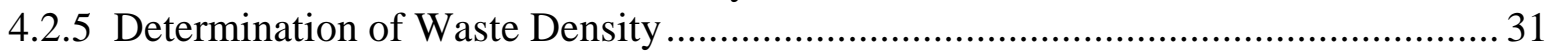

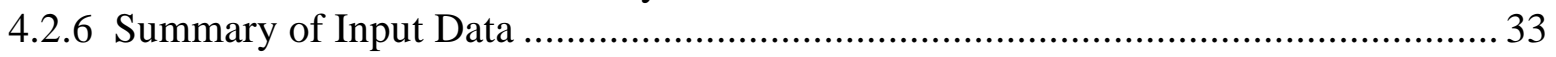

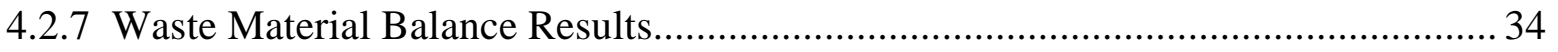

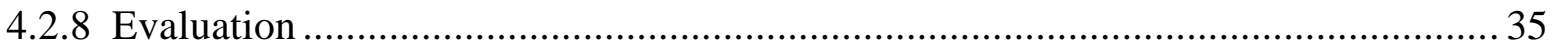

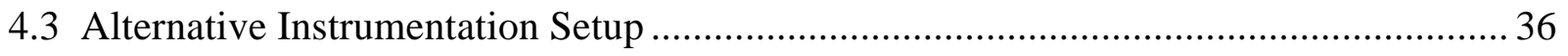

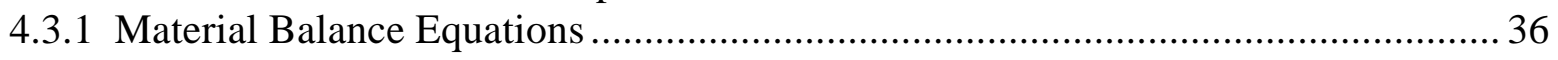

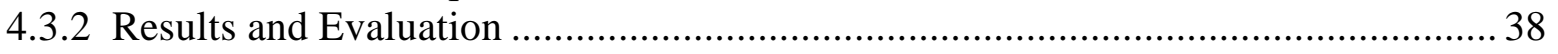

4.3.3 Transfer System Implications of Alternative Instrumentation Setup .......................... 39

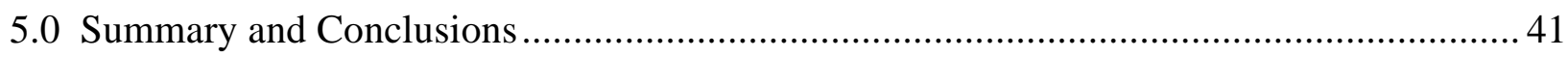

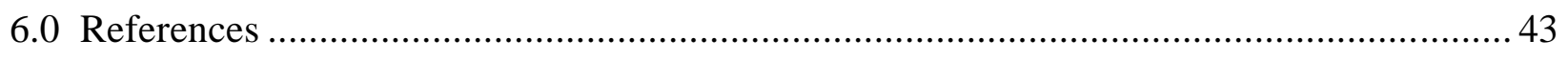

Appendix A: The Micro Motion TSM Calibration System .................................................. A.1

Appendix B: Traceability and Uncertainty Analysis for a Calibration Process for Flowmeters, Using Coriolis Flowmeters as Reference .................................. B.1 


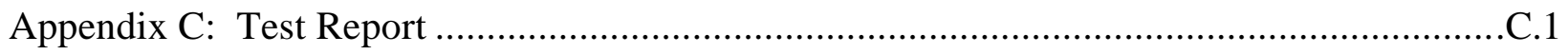

Appendix D: Informal Collection of Users of Micro Motion Meters in Radioactive Environments and/or Nuclear Applications .................................................... D.1

Appendix E: Materials in CMF 100 and T 100 Micro Motion Meters ......................................... 1 


\section{Figures}

2.1 Waste Transfer System and Associated Instrumentation ........................................... 3

3.1 Percent Error as a Function of the Percent of Full-Scale Mass Flow Rate for Elite Series Micro Motion Flow Meters .............................................................. 15

3.2 Zeolite Slurry Mass Flow Rate and Percent Error Between Test Tank Load Cell Readings and Integral of 1-in. Micro Motion CMF Meter

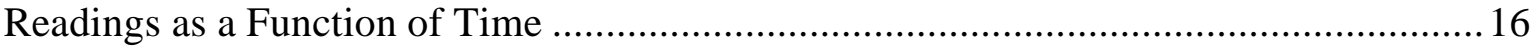

4.1 Tank Wall Waste Retention During Transfer 1 ........................................................ 28

4.2 Tank Wall Waste Retention After Transfer 2 ........................................................... 28

4.3 Waste Surface Irregularity Effect on Height Measurements.......................................28

4.4 Measured and Predicted Supernatant Chemical Concentrations for AN-105 ...................332

4.5 Predicted Dry Solid Concentration for AN-105 Supernatant Diluted at 56\%

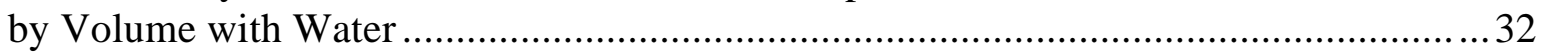

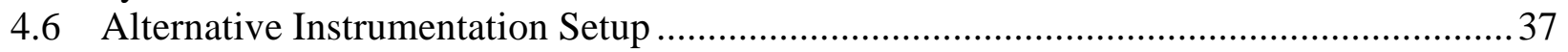

\section{Tables}

$3.1 \quad$ Instrumentation Errors ........................................................................... 22

4.1 Transfer Parameters ..................................................................................... 23

4.2 Instrumentation Material Balance Error for Transfer 1 ..........................................25

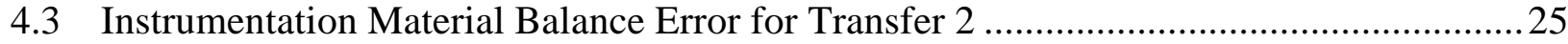

4.4 Instrumentation Material Balance Error for Transfer 3 ............................................. 25

4.5 SY-101 Transfer Volume Comparison ................................................................. 27

4.6 AN-105 first Transfer Parameters......................................................................... 33

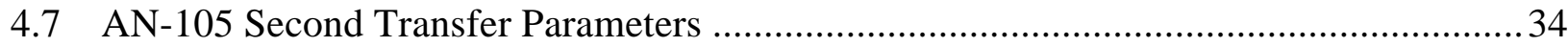

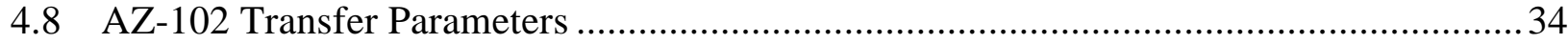

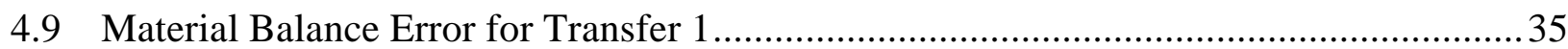

4.10 Material Balance Error for Transfer 2 ................................................................... 35

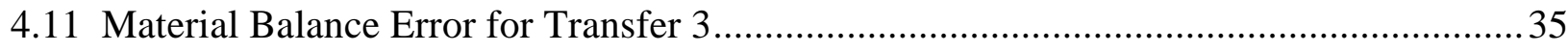

4.12 Pipeline Material Balance Error, All Transfers ......................................................... 38

4.13 Alternative Instrumentation Setup Material Balance Error, All Transfers ...................... 38

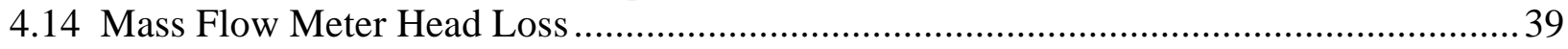




\subsection{Introduction}

The objective of this study was to develop a material balance calculation methodology for variable waste properties and tank conditions to detect waste leaking and misroutings during the double-shell tank (DST) waste pipeline transfer between and within the 200 East and 200 West Areas of the U.S. Department of Energy's Hanford Site. Waste will be transferred from a DST either to another DST or to a waste treatment (vitrification) plant storage tank. This methodology is to address a Final Safety Analysis Report's recommendation to be able to perform material balance calculations as a backup method to pit leak detectors.

The only difference between the original report, PNNL-13485, released in March 2001, and this current report, PNNL-13485 Rev. 1, is that Rev. 1 contains additional information on Micro Motion Coriolis mass flow meters. Otherwise, the two reports are identical.

The volume-based material balance historically has been used successfully for constant waste properties and tank conditions. However, volume-based material balance is not satisfactory for cases with variable waste properties and tank conditions. This limitation is evident for the waste transfer from Tank 241-SY-101 (SY-101) to Tank 241-SY-102 (SY-102) performed in December 1999. In this case, the estimated amount of waste transferred to SY-102 based on SY-101 waste depth changes is $23 \%$ greater than the amount estimated by waste flow discharge measured in the pipeline (Mahoney et al. 2000). The main causes of this large discrepancy are attributed to waste density changes due to chemical reactions, gas release, and the existence of a crust and its changes during the waste transfer. This clearly illustrates that a material balance methodology must be based on conservation of mass to handle variable waste properties and tank conditions.

Thus we developed a material balance assessment methodology for variable waste properties and tank conditions. We performed the following:

- Developed material balance equations for variable waste properties and tank conditions

- Evaluated effect of instrumentation errors only on material balance accuracy with constant waste properties and tank conditions

- Identified possible approaches to address factors affecting waste properties and tank conditions such as chemical reactions, gas release, dissolution of a crust, and the ability to measure waste level when the mixer pumps are running

- Evaluated effects of variable waste properties and tank conditions on material balance accuracy

- Developed an alternative waste instrumentation setup.

We applied the developed material balance equations to three tank waste transfers to demonstrate how this material balance methodology can be used to determine waste leaking and misrouting:

- Tank 241-AN-105 first liquid waste transfer

- Tank 241-AN-105 second liquid waste transfer 
- $\quad$ Tank 241-AZ-102 slurry waste transfer.

In Section 2, we derive the material balance equations for variable waste property and tank conditions. Section 3 describes their three application conditions. Section 4 presents application results on material balance errors due to 1) the measurement instrumentation errors under the constant waste property and tank conditions and 2) variable waste properties and tank conditions. We also present an alternative measurement instrumentation setup and its benefits in this section. Summary and conclusions are presented in Section 5, and cited references are listed in Section 6.

Appendixes A through E contain additional description on the Micro Motion Coriolis mass flow meters. Appendix A describes Micro Motion's calibration system, while Appendix B describes its own traceability and uncertainty analysis on the calibration process. Appendix $\mathrm{C}$ contains a report of Enron/Northern Natural Gas describing their Coriolis meter installation and calibration work. A partial list of users of Micro Motion meters in radioactive nuclear environments is provided in Appendix D. Appendix E lists material of the Coriolis mass flow meter 100 and T 100 Micro Motion meters. 


\subsection{Development of Material Balance Equations}

The three instrumentation setups assigned are described in Section 2.1. General material balance equations based on conservation of mass to handle variable waste properties and tank conditions are derived and their integration forms presented in Section 2.2. Simplified material balance equations for constant waste properties and tank conditions are derived from the general material balance equations and presented in Section 2.3.

\subsection{Instrumentation Setups}

The waste transfers to be evaluated are 1) low-activity waste feed (supernatant with minimal solids) with and without in-line dilution and 2) high-activity waste feed with no in-line dilution. The waste transfer system consists of a waste feed tank, a diluent (water) supply source (water tank or water pipe), waste transfer pipeline, and a waste receiver tank, as shown in Figure 2.1.

The material balance evaluation assumed that the instrumentation consists of one of the following three assigned configurations (see Figure 2.1):

- Measurement A: Tank waste levels $\left(\mathrm{H}_{\mathrm{F}}\right.$ and $\left.\mathrm{H}_{\mathrm{R}}\right)$ and diluent flow meter $\left(\rho_{\mathrm{W}} \mathrm{Q}_{\mathrm{W}}\right.$ or $\left.\mathrm{Q}_{\mathrm{W}}\right)$

- Measurement B: Flow meter $\left(\rho_{\mathrm{FW}} \mathrm{Q}_{\mathrm{FW}}\right.$ or $\left.\mathrm{Q}_{\mathrm{FW}}\right)$ for diluted waste coming from the feed tank and waste tank level $\left(\mathrm{H}_{\mathrm{R}}\right)$ in the receiver tank

- Measurement $\mathrm{C}$ : Tank waste levels $\left(\mathrm{H}_{\mathrm{F}}\right.$ and $\left.\mathrm{H}_{\mathrm{R}}\right)$ and diluent tank level $\left(\mathrm{H}_{\mathrm{W}}\right)$.

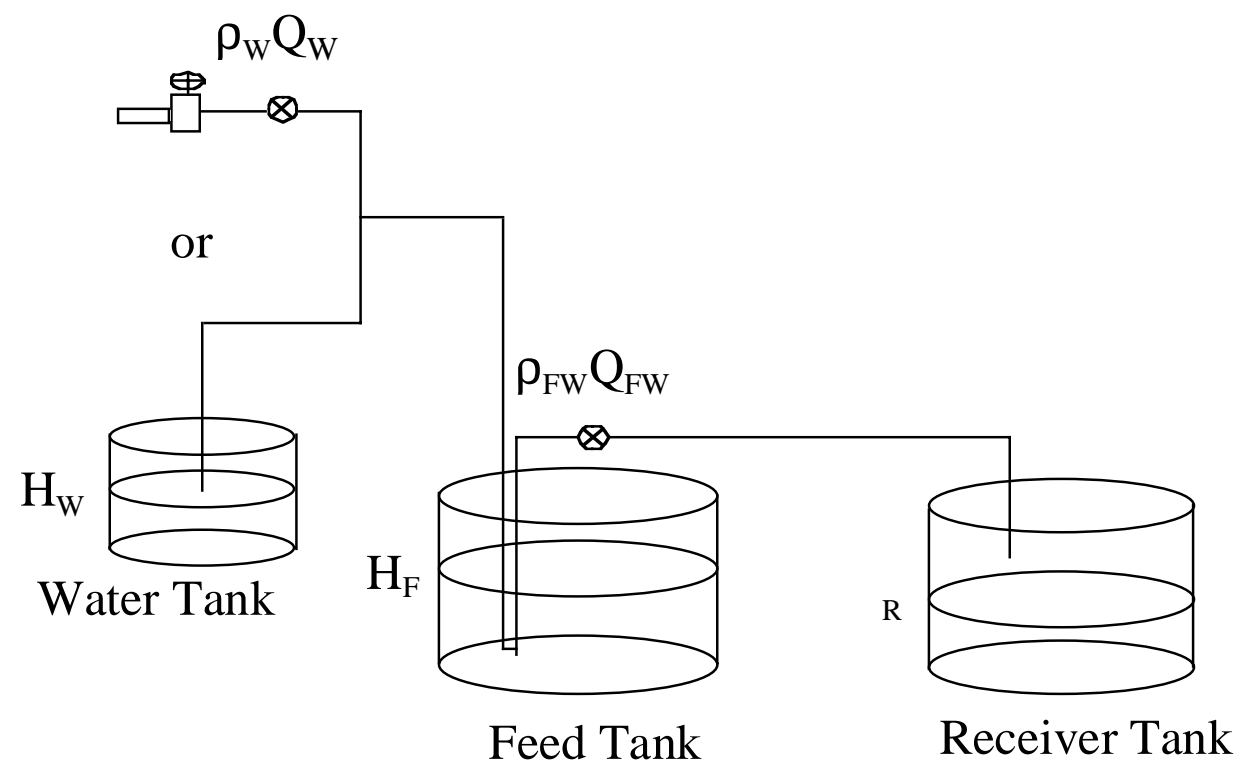

Figure 2.1. Waste Transfer System and Associated Instrumentation 
where

$$
\begin{aligned}
& H=\text { height } \\
& Q=\text { flow rate } \\
& \rho=\text { density }
\end{aligned}
$$

and subscripts $\mathrm{F}, \mathrm{R}, \mathrm{W}=$ those of the feed tank, receiver tank, and water, respectively.

\subsection{Material Balance Equations}

Material balance equations were developed for each instrumentation setup. The assumptions made to develop the equations are given in Section 2.2.1. The general material balance equations are presented in Section 2.2.2, and their integration forms are given in Section 2.2.3.

\subsubsection{Assumptions}

To derive the material balance equations based on conservation of mass, we assumed

- variable waste and liquid densities

- variable water and waste feed flow rates

- variable cross sectional areas of water, feed, and receiver tanks

- water or waste initially contained in a receiver tank.

\subsubsection{General Material Balance Equations}

In the simplest terms, the conservation of mass states that

Loss of Feed Waste Mass + Water Mass Added $=$ Mass Being Transferred

$$
=\text { Gain of Receiver Tank Waste Mass }
$$

or

Amount of Mass Error $=$ Mass Being Transferred - Receiver Tank Mass.

We applied this conservation of mass to the three instrumentation setups.

- Measurement A (known $\left.\mathbf{Q}_{\mathrm{w}}, \mathrm{H}_{\mathrm{F}}, \mathrm{H}_{\mathrm{R}}\right)$

Material balance error (including leaking or misroutings) over the transfer period may be expressed by

$$
\begin{aligned}
& E=\int_{t=t_{0}}^{t=t_{1}} \rho_{w} Q_{w} d t-\int_{H_{F}=H_{F}}^{H_{F}=H_{F 1}} A_{F} \rho_{F} d H_{F}-\left[\rho_{P} \frac{\pi D_{P}^{2} L_{P}}{4}+\int_{t=t_{0}}^{t=t_{1}} \rho_{F W} Q_{F W} d t-\int_{t=t_{0}}^{t=t_{1}} \rho_{P D} Q_{P D} d t\right] \\
& -\left[\begin{array}{l}
H_{R}=H_{R 1} \\
\int_{R}=H_{R} 0
\end{array} A_{R} \rho_{R} d H_{R}-A_{R} \rho_{R 0} H_{R 0}\right]
\end{aligned}
$$

where 


$$
\begin{aligned}
& A=\text { tank cross sectional area } \\
& D_{P}=\text { pipe diameter } \\
& L_{P}=\text { pipeline length }
\end{aligned}
$$

subscripts F0 and F1 = Feed tank values at the beginning and end of transfer

subscript PD = values at the downstream end of the pipeline

subscripts $\mathrm{R} 0$ and $\mathrm{R} 1=$ receiver tank values at the beginning and end of transfer

$\mathrm{t}, \mathrm{t}_{0}, \mathrm{t}_{1}=$ time, time transfer began, and time transfer ended.

\section{- Measurement B (known $\left.\mathbf{Q}_{\mathrm{FW}}, \mathrm{H}_{\mathrm{R}}\right)$}

Material balance error (including leaking or misroutings) over the transfer period may be expressed by

$$
\begin{aligned}
& \mathrm{E}=\int_{\mathrm{t}=\mathrm{t}_{0}}^{\mathrm{t}=\mathrm{t}_{1}} \rho_{\mathrm{FW}} \mathrm{Q}_{\mathrm{FW}} \mathrm{dt}-\left[\rho_{\mathrm{P}} \frac{\pi \mathrm{D}_{\mathrm{P}}^{2} \mathrm{~L}_{\mathrm{P}}}{4}+\int_{\mathrm{t}=\mathrm{t}_{0}}^{\mathrm{t}=\mathrm{t}_{1}} \rho_{\mathrm{FW}} \mathrm{Q}_{\mathrm{FW}} \mathrm{dt}-\int_{\mathrm{t}=\mathrm{t}_{0}}^{\mathrm{t}=\mathrm{t}_{\mathrm{t}}} \rho_{\mathrm{PD}} \mathrm{Q}_{\mathrm{PD}} \mathrm{dt}\right] \\
& -\left[\begin{array}{l}
\mathrm{H}_{\mathrm{R}}=\mathrm{H}_{\mathrm{R} 1} \\
\int_{\mathrm{R}}=\mathrm{H}_{\mathrm{R} 0}
\end{array} \mathrm{~A}_{\mathrm{R}} \rho_{\mathrm{R}} \mathrm{dH}_{\mathrm{R}}-\mathrm{A}_{\mathrm{R}} \rho_{\mathrm{R} 0} \mathrm{H}_{\mathrm{R} 0}\right]
\end{aligned}
$$

\section{- Measurement $\mathrm{C}\left(\right.$ known $\left.\mathrm{H}_{\mathrm{W}}, \mathrm{H}_{\mathrm{F}}, \mathrm{H}_{\mathrm{R}}\right)$}

Material balance error (including leaking or misroutings) over the transfer period may be expressed by

$$
\begin{aligned}
& \mathrm{E}=-\int_{\mathrm{H}_{\mathrm{W}}=\mathrm{H}_{\mathrm{W}}}^{\mathrm{H}_{\mathrm{W}}=\mathrm{H}_{\mathrm{W} 1}} \mathrm{~A}_{\mathrm{W}} \rho_{\mathrm{W}} \mathrm{dH} \mathrm{H}_{\mathrm{W}}-\int_{\mathrm{H}_{\mathrm{F}}=\mathrm{H}_{\mathrm{F}}}^{\mathrm{H}_{\mathrm{F}}=\mathrm{H}_{\mathrm{F} 1}} \mathrm{~A}_{\mathrm{F}} \rho_{\mathrm{F}} \mathrm{dH} \mathrm{H}_{\mathrm{F}}-\left[\rho_{\mathrm{P}} \frac{\pi \mathrm{D}_{\mathrm{P}}^{2} \mathrm{~L}_{\mathrm{P}}}{4}+\int_{\mathrm{t}=\mathrm{t}_{0}}^{\mathrm{t}=\mathrm{t}_{1}} \rho_{\mathrm{FW}} \mathrm{Q}_{\mathrm{FW}} \mathrm{dt}-\int_{\mathrm{t}=\mathrm{t}_{0}}^{\mathrm{t}=\mathrm{t}_{1}} \rho_{\mathrm{PD}} \mathrm{Q}_{\mathrm{PD}} \mathrm{dt}\right] \\
& -\left[\begin{array}{c}
\mathrm{H}_{\mathrm{R}}=\mathrm{H}_{\mathrm{R} 1} \\
\int_{\mathrm{H}_{\mathrm{R}}=0} \mathrm{~A}_{\mathrm{R}} \rho_{\mathrm{R}} \mathrm{dH}_{\mathrm{R}}-\mathrm{A}_{\mathrm{R}} \rho_{\mathrm{R} 0} \mathrm{H}_{\mathrm{R} 0}
\end{array}\right]
\end{aligned}
$$

Equations (2.1) through (2.3) are general equations for variable waste properties and tank conditions. Waste densities, tank cross-sectional areas, and flow rates can vary during the waste transfer in these equations. In Section 4.2, we describe how these terms may be determined and apply them to the three transfer cases studied. The integral forms in Equations (2.1) through (2.3) may be calculated by as given in the following section.

\subsubsection{Integration Forms}

\section{- Integration forms for water tank mass flow, $\rho_{\mathrm{w}} Q_{\mathrm{w}}$ in Equation (2.1)}

Water mass, $\mathrm{M}_{\mathrm{W}}$, added to the waste is

$$
\mathrm{M}_{\mathrm{W}}=\int_{\mathrm{t}=\mathrm{t}_{0}}^{\mathrm{t}=\mathrm{t}_{1}} \rho_{\mathrm{W}} \mathrm{Q}_{\mathrm{W}} \mathrm{dt}
$$


With Simpson's rule $(\mathrm{n}=$ even integer),

$$
\begin{aligned}
& \mathrm{M}_{\mathrm{W}}=\frac{\Delta \mathrm{t}}{3}\left(\rho_{\mathrm{W} 0} \mathrm{Q}_{\mathrm{W} 0}+4 \rho_{\mathrm{W} 0-\Delta \mathrm{t}} \mathrm{Q}_{\mathrm{W} 0-\Delta \mathrm{t}}+2 \rho_{\mathrm{W} 0-2 \Delta \mathrm{t}} \mathrm{Q}_{\mathrm{W} 0-2 \Delta \mathrm{t}}+4 \rho_{\mathrm{W} 0-3 \Delta \mathrm{t}} \mathrm{Q}_{\mathrm{W} 0-3 \Delta \mathrm{t}}+. .\right. \\
& \left.\ldots+2 \rho_{\mathrm{W} 0-(\mathrm{n}-2) \Delta \mathrm{t}} \mathrm{Q}_{\mathrm{W} 0-(\mathrm{n}-2) \Delta \mathrm{t}}+4 \rho_{\mathrm{W} 0-(\mathrm{n}-1) \Delta \mathrm{t}} \mathrm{Q}_{\mathrm{W} 0-(\mathrm{n}-1) \Delta \mathrm{t}}+\rho_{\mathrm{W} 1} \mathrm{Q}_{\mathrm{W} 1}\right) \\
& \qquad \mathrm{t}=\frac{\mathrm{t}_{1}-\mathrm{t}_{0}}{\mathrm{n}}
\end{aligned}
$$

With the trapezoidal rule $(\mathrm{n}=$ integer $)$

$$
\begin{aligned}
& \mathrm{M}_{\mathrm{W}}=\frac{\Delta \mathrm{t}}{2}\left(\rho_{\mathrm{W} 0} \mathrm{Q}_{\mathrm{W} 0}+2 \rho_{\mathrm{W} 0-\Delta \mathrm{t}} \mathrm{Q}_{\mathrm{W} 0-\Delta \mathrm{t}}+2 \rho_{\mathrm{W} 0-2 \Delta \mathrm{t}} \mathrm{Q}_{\mathrm{W} 0-2 \Delta \mathrm{t}}+2 \rho_{\mathrm{W} 0-3 \Delta \mathrm{t}} \mathrm{Q}_{\mathrm{W} 0-3 \Delta \mathrm{t}}+. .\right. \\
& \left.\ldots+2 \rho_{\mathrm{W} 0-(\mathrm{n}-2) \Delta \mathrm{t}} \mathrm{Q}_{\mathrm{W} 0-(\mathrm{n}-2) \Delta \mathrm{t}}+2 \rho_{\mathrm{W} 0-(\mathrm{n}-1) \Delta \mathrm{t}} \mathrm{Q}_{\mathrm{W} 0-(\mathrm{n}-1) \Delta \mathrm{t}}+\rho_{\mathrm{W} 1} \mathrm{Q}_{\mathrm{W} 1}\right)
\end{aligned}
$$

Note that Simpson's rule produces more accurate integration values than the Trapezoidal rule does, but the latter may be easier to implement.

\section{- Integration Forms for Water Tank Height, $H_{W}$ in Equations (2.2) and (2.3)}

Water mass lost in a water tank, $\mathrm{M}_{\mathrm{W}}$ is

$$
M_{W}=-\int_{H_{\mathrm{W}}=\mathrm{H}_{\mathrm{w} 0}}^{\mathrm{H}_{\mathrm{w}}=\mathrm{H}_{\mathrm{W} 1}} \mathrm{~A}_{\mathrm{W}} \rho_{\mathrm{W}} \mathrm{dH} \mathrm{H}_{\mathrm{W}}
$$

With Simpson's rule:

$$
\begin{aligned}
& \mathrm{M}_{\mathrm{W}}=\frac{\Delta \mathrm{H}}{3}\left(\mathrm{~A}_{\mathrm{W} 0} \rho_{\mathrm{W} 0}+4 \mathrm{~A}_{\mathrm{W} 0-\Delta \mathrm{H}} \rho_{\mathrm{W} 0-\Delta \mathrm{H}}+2 \mathrm{~A}_{\mathrm{W} 0-2 \Delta \mathrm{H}} \rho_{\mathrm{W} 0-2 \Delta \mathrm{H}}+4 \mathrm{~A}_{\mathrm{W} 0-3 \Delta \mathrm{H}} \rho_{\mathrm{W} 0-3 \Delta \mathrm{H}}+\ldots\right. \\
& \left.+2 \mathrm{~A}_{\mathrm{W} 0-(\mathrm{n}-2) \Delta \mathrm{H}} \rho_{\mathrm{W} 0-(\mathrm{n}-2) \Delta \mathrm{H}}+4 \mathrm{~A}_{\mathrm{W} 0-(\mathrm{n}-1) \Delta \mathrm{H}} \rho_{\mathrm{W} 0-(\mathrm{n}-1) \Delta \mathrm{H}}+\mathrm{A}_{\mathrm{W} 1} \rho_{\mathrm{W} 1}\right) \\
& \Delta H=\frac{H_{F 0}-H_{F 1}}{n}
\end{aligned}
$$

With the trapezoidal rule:

$$
\begin{aligned}
& \mathrm{M}_{\mathrm{W}}=\frac{\Delta \mathrm{H}}{2}\left(\mathrm{~A}_{\mathrm{W} 0} \rho_{\mathrm{W} 0}+2 \mathrm{~A}_{\mathrm{W} 0-\Delta \mathrm{H}} \rho_{\mathrm{W} 0-\Delta \mathrm{H}}+2 \mathrm{~A}_{\mathrm{W} 0-2 \Delta \mathrm{H}} \rho_{\mathrm{W} 0-2 \Delta \mathrm{H}}+2 \mathrm{~A}_{\mathrm{W} 0-3 \Delta \mathrm{H}} \rho_{\mathrm{W} 0-3 \Delta \mathrm{H}}+\ldots\right. \\
& \left.+2 \mathrm{~A}_{\mathrm{W} 0-(\mathrm{n}-2) \Delta \mathrm{H}} \rho_{\mathrm{W} 0-(\mathrm{n}-2) \Delta \mathrm{H}}+2 \mathrm{~A}_{\mathrm{W} 0-(\mathrm{n}-1) \Delta \mathrm{H}} \rho_{\mathrm{W} 0-(\mathrm{n}-1) \Delta \mathrm{H}}+\mathrm{A}_{\mathrm{W} 1} \rho_{\mathrm{W} 1}\right)
\end{aligned}
$$

- Integration Forms for Feed Tank Height, $H_{F}$ in Equations (2.1) and (2.3)

Waste mass lost in a feed tank, $\mathrm{M}_{\mathrm{F}}$ is

$$
M_{F}=-\int_{H_{F}=H_{F} 0}^{H_{F}=H_{F 1}} A_{F} \rho_{F} d H_{F}
$$


With Simpson's Rule,

$$
\begin{aligned}
& \mathrm{M}_{\mathrm{F}}=-\frac{\Delta \mathrm{H}_{\mathrm{F}}}{3}\left(\mathrm{~A}_{\mathrm{F} 0} \rho_{\mathrm{F} 0}+4 \mathrm{~A}_{\mathrm{F} 0-\Delta \mathrm{H}} \rho_{\mathrm{F} 0-\Delta \mathrm{H}}+2 \mathrm{~A}_{\mathrm{F} 0-2 \Delta \mathrm{H}} \rho_{\mathrm{F} 0-2 \Delta \mathrm{H}}+4 \mathrm{~A}_{\mathrm{F} 0-3 \Delta \mathrm{H}} \rho_{\mathrm{F} 0-3 \Delta \mathrm{H}}+\ldots\right. \\
& \left.+2 \mathrm{~A}_{\mathrm{F} 0-(\mathrm{n}-2) \Delta \mathrm{H}} \rho_{\mathrm{F} 0-(\mathrm{n}-2) \Delta \mathrm{H}}+4 \mathrm{~A}_{\mathrm{F} 0-(\mathrm{n}-1) \Delta \mathrm{H}} \rho_{\mathrm{F} 0-(\mathrm{n}-1) \Delta \mathrm{H}}+\mathrm{A}_{\mathrm{F} 1} \rho_{\mathrm{F} 1}\right) \\
& \quad \Delta \mathrm{H}_{\mathrm{F}}=\frac{\mathrm{H}_{\mathrm{F} 0}-\mathrm{H}_{\mathrm{F} 1}}{\mathrm{n}}
\end{aligned}
$$

With the Trapezoidal rule:

$$
\begin{aligned}
& \mathrm{M}_{\mathrm{F}}=-\frac{\Delta \mathrm{H}_{\mathrm{F}}}{2}\left(\mathrm{~A}_{\mathrm{F} 0} \rho_{\mathrm{F} 0}+2 \mathrm{~A}_{\mathrm{F} 0-\Delta \mathrm{H}} \rho_{\mathrm{F} 0-\Delta \mathrm{H}}+2 \mathrm{~A}_{\mathrm{F} 0-2 \Delta \mathrm{H}} \rho_{\mathrm{F} 0-2 \Delta \mathrm{H}}+2 \mathrm{~A}_{\mathrm{F} 0-3 \Delta} \rho_{\mathrm{F} 0-3 \Delta \mathrm{H}}+\ldots\right. \\
& \left.+2 \mathrm{~A}_{\mathrm{F} 0-(\mathrm{n}-2) \Delta \mathrm{H}} \rho_{\mathrm{F} 0-(\mathrm{n}-2) \Delta \mathrm{H}}+2 \mathrm{~A}_{\mathrm{F} 0-(\mathrm{n}-1) \Delta \mathrm{H}} \rho_{\mathrm{F} 0-(\mathrm{n}-1) \Delta \mathrm{H}}+\mathrm{A}_{\mathrm{Fl}} \rho_{\mathrm{Fl}}\right)
\end{aligned}
$$

- Integration Forms for Pipeflow Discharge, $\rho_{F W} Q_{F W}$ in Equations (2.1) (2.3)

Diluted waste mass in at the pipeline upstream end, $\mathrm{M}_{\mathrm{FW}}$ is

$$
M_{F W}=\int_{t=t_{0}}^{t=t_{1}} \rho_{F W} Q_{F W} d t
$$

With Simpson's rule,

$$
\begin{aligned}
& \mathrm{M}_{\mathrm{FW}}=\frac{\Delta \mathrm{t}}{3}\left(\rho_{\mathrm{FW} 0} \mathrm{Q}_{\mathrm{FW} 0}+4 \rho_{\mathrm{FW} 0-\Delta \mathrm{t}} \mathrm{Q}_{\mathrm{FW} 0-\Delta \mathrm{t}}+2 \rho_{\mathrm{FW} 0-2 \Delta \mathrm{t}} \mathrm{Q}_{\mathrm{FW} 0-2 \Delta \mathrm{t}}+4 \rho_{\mathrm{FW} 0-3 \Delta \mathrm{t}} \mathrm{Q}_{\mathrm{FW} 0-3 \Delta \mathrm{t}}+. .\right. \\
& \left.\ldots+2 \rho_{\mathrm{FW} 0-(\mathrm{n}-2) \Delta \mathrm{t}} \mathrm{Q}_{\mathrm{FW} 0-(\mathrm{n}-2) \Delta \mathrm{t}}+4 \rho_{\mathrm{FW} 0-(\mathrm{n}-1) \Delta \mathrm{t}} \mathrm{Q}_{\mathrm{FW} 0-(\mathrm{n}-1) \Delta \mathrm{t}}+\rho_{\mathrm{FW} 1} \mathrm{Q}_{\mathrm{FW} 1}\right) \\
& \quad \Delta \mathrm{t}=\frac{\mathrm{t}_{1}-\mathrm{t}_{0}}{\mathrm{n}}
\end{aligned}
$$

With the Trapezoidal rule,

$$
\begin{aligned}
& \mathrm{M}_{\mathrm{FW}}=\frac{\Delta \mathrm{t}}{2}\left(\rho_{\mathrm{FW} 0} \mathrm{Q}_{\mathrm{FW} 0}+2 \rho_{\mathrm{FW} 0-\Delta \mathrm{t}} \mathrm{Q}_{\mathrm{FW} 0-\Delta \mathrm{t}}+2 \rho_{\mathrm{FW} 0-2 \Delta \mathrm{t}} \mathrm{Q}_{\mathrm{FW} 0-2 \Delta \mathrm{t}}+2 \rho_{\mathrm{FW} 0-3 \Delta \mathrm{t}} \mathrm{Q}_{\mathrm{FW} 0-3 \Delta \mathrm{t}}+. .\right. \\
& \left.\ldots+2 \rho_{\mathrm{FW} 0-(\mathrm{n}-2) \Delta \mathrm{t}} \mathrm{Q}_{\mathrm{FW} 0-(\mathrm{n}-2) \Delta \mathrm{t}}+2 \rho_{\mathrm{FW} 0-(\mathrm{n}-1) \Delta \mathrm{t}} \mathrm{Q}_{\mathrm{FW} 0-(\mathrm{n}-1) \Delta \mathrm{t}}+\rho_{\mathrm{FW} 1} \mathrm{Q}_{\mathrm{FW} 1}\right)
\end{aligned}
$$

\section{- Integration Forms for Pipeflow Discharge, $\rho_{P D} Q_{P D}$ in Equations (2.1) (2.3)}

Diluted waste mass at the pipeline downstream end, $\mathrm{M}_{\mathrm{PD}}$ is

$$
\mathrm{M}_{\mathrm{PD}}=\int_{\mathrm{t}=\mathrm{t}_{0}}^{\mathrm{t}=\mathrm{t}_{1}} \rho_{\mathrm{PD}} \mathrm{Q}_{\mathrm{PD}} \mathrm{dt}
$$


With Simpson's rule,

$$
\begin{aligned}
& \mathrm{M}_{\mathrm{PD}}=\frac{\Delta \mathrm{t}}{3}\left(\rho_{\mathrm{PD} 0} \mathrm{Q}_{\mathrm{PD} 0}+4 \rho_{\mathrm{PD} 0-\Delta \mathrm{t}} \mathrm{Q}_{\mathrm{PD} 0-\Delta \mathrm{t}}+2 \rho_{\mathrm{PD} 0-2 \Delta \mathrm{t}} \mathrm{Q}_{\mathrm{PD} 0-2 \Delta \mathrm{t}}+4 \rho_{\mathrm{PD} 0-3 \Delta \mathrm{t}} \mathrm{Q}_{\mathrm{PD} 0-3 \Delta \mathrm{t}}+. .\right. \\
& \left.\ldots+2 \rho_{\mathrm{PD} 0-(\mathrm{n}-2) \Delta \mathrm{t}} \mathrm{Q}_{\mathrm{PD} 0-(\mathrm{n}-2) \Delta \mathrm{t}}+4 \rho_{\mathrm{PD} 0-(\mathrm{n}-1) \Delta \mathrm{t}} \mathrm{Q}_{\mathrm{PD} 0-(\mathrm{n}-1) \Delta \mathrm{t}}+\rho_{\mathrm{PD} 1} \mathrm{Q}_{\mathrm{PD} 1}\right) \\
& \quad \Delta \mathrm{t}=\frac{\mathrm{t}_{1}-\mathrm{t}_{0}}{\mathrm{n}}
\end{aligned}
$$

With the Trapezoidal rule:

$$
\begin{aligned}
& \mathrm{M}_{\mathrm{PD}}=\frac{\Delta \mathrm{t}}{2}\left(\rho_{\mathrm{PD} 0} \mathrm{Q}_{\mathrm{PD} 0}+2 \rho_{\mathrm{PD} 0-\Delta \mathrm{t}} \mathrm{Q}_{\mathrm{PD} 0-\Delta \mathrm{t}}+2 \rho_{\mathrm{PD} 0-2 \Delta \mathrm{t}} \mathrm{Q}_{\mathrm{PD} 0-2 \Delta \mathrm{t}}+2 \rho_{\mathrm{PD} 0-3 \Delta \mathrm{t}} \mathrm{Q}_{\mathrm{PD} 0-3 \Delta \mathrm{t}}+. .\right. \\
& \left.\ldots+2 \rho_{\mathrm{PD} 0-(\mathrm{n}-2) \Delta \mathrm{t}} \mathrm{Q}_{\mathrm{PD} 0-(\mathrm{n}-2) \Delta \mathrm{t}}+2 \rho_{\mathrm{PD} 0-(\mathrm{n}-1) \Delta \mathrm{t}} \mathrm{Q}_{\mathrm{PD} 0-(\mathrm{n}-1) \Delta \mathrm{t}}+\rho_{\mathrm{PD} 1} \mathrm{Q}_{\mathrm{PD} 1}\right)
\end{aligned}
$$

- Integration Forms for Receiver Tank Height, $H_{R}$ in Equations (2.1) (2.3)

Waste mass gained in a receiver tank, $M_{R}$, is

$$
M_{R}=\int_{H_{R}=H_{R} 0}^{H_{R}=H_{R 1}} A_{R} \rho_{R} d H_{R}
$$

With Simpson's rule:

$$
\begin{aligned}
& \mathrm{M}_{\mathrm{R}}=\frac{\Delta \mathrm{H}_{\mathrm{R}}}{3}\left(\mathrm{~A}_{\mathrm{R} 0} \rho_{\mathrm{R} 0}+4 \mathrm{~A}_{\mathrm{R} 0-\Delta \mathrm{H}} \rho_{\mathrm{R} 0-\Delta \mathrm{H}}+2 \mathrm{~A}_{\mathrm{R} 0-2 \Delta \mathrm{H}} \rho_{\mathrm{R} 0-2 \Delta \mathrm{H}}+4 \mathrm{~A}_{\mathrm{R} 0-3 \Delta \mathrm{H}} \rho_{\mathrm{R} 0-3 \Delta \mathrm{H}}+\ldots\right. \\
& \left.+2 \mathrm{~A}_{\mathrm{R} 0-(\mathrm{n}-2) \Delta \mathrm{H}} \rho_{\mathrm{R} 0-(\mathrm{n}-2) \Delta \mathrm{H}}+4 \mathrm{~A}_{\mathrm{R} 0-(\mathrm{n}-1) \Delta \mathrm{H}} \rho_{\mathrm{R} 0-(\mathrm{n}-1) \Delta \mathrm{H}}+\mathrm{A}_{\mathrm{R} 1} \rho_{\mathrm{R} 1}\right) \\
& \quad \Delta \mathrm{H}_{\mathrm{R}}=\frac{\mathrm{H}_{\mathrm{R} 0}-\mathrm{H}_{\mathrm{R} 1}}{\mathrm{n}}
\end{aligned}
$$

With the Trapezoidal rule:

$$
\begin{aligned}
& \mathrm{M}_{\mathrm{R}}=\frac{\Delta \mathrm{H}_{\mathrm{R}}}{2}\left(\mathrm{~A}_{\mathrm{R} 0} \rho_{\mathrm{R} 0}+2 \mathrm{~A}_{\mathrm{R} 0-\Delta \mathrm{H}} \rho_{\mathrm{R} 0-\Delta \mathrm{H}}+2 \mathrm{~A}_{\mathrm{R} 0-2 \Delta \mathrm{H}} \rho_{\mathrm{R} 0-2 \Delta \mathrm{H}}+2 \mathrm{~A}_{\mathrm{R} 0-3 \Delta \mathrm{H}} \rho_{\mathrm{R} 0-3 \Delta \mathrm{H}}+\ldots\right. \\
& \left.+2 \mathrm{~A}_{\mathrm{R} 0-(\mathrm{n}-2) \Delta \mathrm{H}} \rho_{\mathrm{R} 0-(\mathrm{n}-2) \Delta \mathrm{H}}+2 \mathrm{~A}_{\mathrm{R} 0-(\mathrm{n}-1) \Delta \mathrm{H}} \rho_{\mathrm{R} 0-(\mathrm{n}-1) \Delta \mathrm{H}}+\mathrm{A}_{\mathrm{R} 1} \rho_{\mathrm{R} 1}\right)
\end{aligned}
$$

Time varying values of the water mass flow rate and those values in the water, feed, and receiver tanks must be estimated prior to the waste transfer, while those values in the pipeline may be measured during the transfer period to calculate these integrations. 


\subsection{Simplified Material Balance Equations under Constant Waste Properties and Tank Conditions}

For

- constant densities of $\rho_{\mathrm{w}}, \rho_{\mathrm{F}}, \rho_{\mathrm{R} 0}$, and $\rho_{\mathrm{R} 1}$

- constant $\mathrm{Q}_{\mathrm{w}}$

- no solids settling within a pipeline, and

- $\quad \rho_{\mathrm{FW}} \mathrm{Q}_{\mathrm{FW}}=\rho_{\mathrm{PD}} \mathrm{Q}_{\mathrm{PD}}=\mathrm{cons} \tan \mathrm{t}$

- $\quad$ pipeline flushed at the end to remove the waste from the line, Equation (2.1) becomes

$E=\rho_{\mathrm{W}} \mathrm{Q}_{\mathrm{W}} \mathrm{t}_{1}+\mathrm{A}_{\mathrm{F}} \rho_{\mathrm{F}}\left(\mathrm{H}_{\mathrm{F} 0}-\mathrm{H}_{\mathrm{F} 1}\right)-\mathrm{A}_{\mathrm{R}}\left(\rho_{\mathrm{R} 1} \mathrm{H}_{\mathrm{R} 1}-\rho_{\mathrm{R} 0} \mathrm{H}_{\mathrm{R} 0}\right)$

Similarly, under these conditions, Equations (2.2) and (2.3) become

$\mathrm{E}=\rho_{\mathrm{FW}} \mathrm{Q}_{\mathrm{FW}} \mathrm{t}_{1}-\mathrm{A}_{\mathrm{R}}\left(\rho_{\mathrm{R} 1} \mathrm{H}_{\mathrm{R} 1}-\rho_{\mathrm{R} 0} \mathrm{H}_{\mathrm{R} 0}\right)$

and

$\mathrm{E}=\mathrm{A}_{\mathrm{W}} \rho_{\mathrm{W}}\left(\mathrm{H}_{\mathrm{W} 0}-\mathrm{H}_{\mathrm{W} 1}\right)+\mathrm{A}_{\mathrm{F}} \rho_{\mathrm{F}}\left(\mathrm{H}_{\mathrm{F} 0}-\mathrm{H}_{\mathrm{F} 1}\right)-\mathrm{A}_{\mathrm{R}}\left(\rho_{\mathrm{R} 1} \mathrm{H}_{\mathrm{R} 1}-\rho_{\mathrm{R} 0} \mathrm{H}_{\mathrm{R} 0}\right)$

These simplified mass balance equations (2.22-2.24) were used for the actual applications in this study, as will be discussed in Sections 3 and 4. 


\subsection{Material Balance Applications}

The equations presented in Section 2 are used to evaluate possible material balance errors during Hanford waste transfers. In Section 3.1, the specific waste transfer scenarios evaluated are presented. Section 3.2 presents the instrumentation considered and used, and Section 3.3 summarizes the material balance statistical assessment methodology.

\subsection{Transfer Scenarios}

Three distinctly different transfer scenarios out of Hanford double-shell tanks (DSTs) were considered. They include the transfer and in-line dilution of supernatant liquid, the transfer of diluted settled solids, and the transfer of a mixed slurry. The specific cases considered are listed below.

Transfer 1: The first transfer from Tank 241-AN-105 (AN-105) is expected to remove approximately 584,000 gallons of supernatant liquid from the tank. This waste will be diluted in-line with water at $56 \%$ by volume, yielding a total transfer of approximately 911,400 gallons. The waste will be transferred into Tank 241-AN-102 (AN-102). It is assumed that the water in $\mathrm{AN}-102$ at the time of transfer is 0.305 -m-deep.

Transfer 2: After the supernatant liquid has been removed from AN-105, an in-tank dilution of the settled solids with water is planned at $79 \%$ by volume, after which the tank will be mixed. Data from Herting (1997) indicates that this dilution amount will dissolve the bulk of the soluble solids. The second transfer from AN-105 will be conducted after any remaining solids in the tank have been allowed to settle. The subsequent supernatant liquid transfer is estimated to be approximately 673,000 gallons. Two different receiver tanks are considered. The first is an empty DST, while the second is an empty 46-ft-diameter tank at the vitrification plant.

Transfer 3: Tank 241-AZ-102 (AZ-102) will be mixed before and during transfer. The waste transferred will therefore contain solids at approximately $2 \%$ by volume, as determined from Schreiber (1995) and Bingham et al. (2000). The transfer batch size is 150,000 gallons. Again, two receiver tanks are considered: an empty DST and an empty 25-ft-diameter tank at the vitrification plant.

\subsection{Instrumentation}

Three different instrumentation setups were discussed in Section 2.1. Instrumentation setups $\mathrm{A}$ and $\mathrm{B}$ require both waste flow and surface level measurements, while instrumentation setup $\mathrm{C}$ requires waste and water surface level measurements only.

The options evaluated for obtaining flow rate measurements included Coriolis mass flow meters (CFM), electromagnetic (EM) flow meters, and ultrasonic technologies. Ultrasonic devices are available for measuring both density and fluid velocity within a pipe (Hylton and Bayne 1999; Greenwood and Bamberger 2000), but at its current stage of development, the technology does not appear to provide the accuracy or reliability that is obtained with 
commercially available Coriolis and EM meters. Thus no further discussion of ultrasonic technologies will be presented. EM meters measure only the fluid velocity; therefore, to obtain mass flow measurements, the density must be known or obtained using a second device. CFMs measure both the density and mass flow directly. Section 3.2.2 discusses CFMs and has been expanded in this version of the report (Rev. 1) to meet the client's information needs.

The waste and water surface levels are measured with an Enraf buoyancy gauge. These devices are already employed in the Hanford waste tanks, and Hedengren et al. (2000) note that the Enraf is potentially the most reliable surface level indicator because it is not subject to the buildup of waste deposits, nor does it disturb the surface on which it rests.

\subsubsection{Electromagnetic Flow Meters}

Electromagnetic (EM) flow meters apply Faraday's Law for electromagnetic induction to obtain the velocity of a conductive fluid. As a conductive fluid passes through a magnetic field, a voltage results. The magnitude of the voltage is directly proportional to the fluid velocity, the magnetic field strength, and the pipe diameter. For in-line meters, the magnetic field and the fluid velocity are perpendicular; therefore, the velocity can be described by

$$
\mathrm{U}=\frac{\mathrm{V}}{\mathrm{KBD}}
$$

where $\mathrm{U}$ is the average fluid velocity, $\mathrm{V}$ is the induced voltage, $\mathrm{K}$ is the proportionality constant, $\mathrm{B}$ is the magnetic field strength, and $\mathrm{D}$ is the pipe diameter.

EM meters are not affected by changes in the fluid density or viscosity (direct-current meters have been susceptible to noise resulting from high-viscosity fluids) and result in negligible pressure drop because their flow channel is a short section of straight pipe. Both direct current (DC) and alternating current (AC) EM meters exist. Pulsed-DC systems operate at low frequencies (4 to $7 \mathrm{~Hz}$ ), which allows the electronics to be re-zeroed between the pulses. However, the low sampling frequency of the meters makes them susceptible to noise from the flow of slurries, electrochemical reactions, and high viscosities.

AC EM meters reverse polarity at $60 \mathrm{~Hz}$ and are less susceptible to the noise from the flow of slurries. However, the AC meters do experience zero drift.

Yokogawa manufactures a dual-frequency EM meter that is intended to allow for continual re-zeroing while maintaining immunity to noise created by flowing slurries. The instrument has a carrier frequency of $7 \mathrm{~Hz}$ with a low pass filter and a $72-\mathrm{Hz}$ superimposed high-frequency waveform. The accuracy of these meters is rated at $0.5 \%$ volume flow rate for a flow rate range of $20 \%$ of the span and higher. The accuracy of an EM meter depends on a uniform conductivity over the cross-section of the pipe. Variations in conductivity can occur with incomplete mixing of an added fluid such as in water dilution or stratification of a slurry flow. The stratification of slurries often results in reduced accuracy at higher percentages of the flow range than that specified by the manufacturer. 
The Yokogawa EM meters can be installed in vertical or horizontal orientations. It is recommended that a vertical orientation be used for slurries. Flow should be upward through the vertically oriented meter. Five to 10 pipe diameters of straight pipe are required upstream of the meter, depending on the upstream pipe components.

A 2-in. Yokogawa EM meter, model AE14-Da1A/ff1/FN1, was installed in the temporary transfer line used for the waste transfer and back-dilution operations performed to remediate Tank 241-SY-101 in 1999 and 2000 (Estey 2000). The meter was installed as general service equipment as opposed to safety-class or safety-significant equipment. The meter operated successfully throughout the waste transfer sequences.

Yokogawa has not designed any of their instruments for a radiation environment. The wire insulation used in the sensors is either Teflon or polyvinylchloride (PVC), depending on the model. The meter was selected for the SY-101 application based on successful applications with core sampling trucks, the temporary nature of the application, and the safety class of the equipment.

\subsubsection{Coriolis Mass Flow Meters}

Coriolis meters measure both mass flow rate and density directly with the parameters of mass, time, and length as the bases of all measurements. Mass flow rate measurements are accurate under conditions of changing viscosity, conductivity, density, and temperature of the flowing fluid. The principle of operation is based on Newton's second law.

The sensor contains a flow tube that has a symmetrical flow path lying in a single plane. Common configurations of flow tubes have included D- and U-shaped geometries. Improvements in sensor technology and electronics now allow measurements to be obtained from straight flow tubes. The flow tube is vibrated at the natural frequency of the filled tube using a feedback circuit and drive coil. The vibration is induced at the midpoint of the flow tube such that the resulting motion of the tube is perpendicular to the plane of the flow tube.

As fluid enters the flow tube, it takes on the lateral momentum of the vibrating tube. As the fluid is accelerated in the lateral direction, a force is applied to the wall of the flow tube. As the fluid approaches the exit of the tube, it resists having its lateral motion decreased. The resulting force applied to the tube wall at the downstream side of the flow tube is opposite in direction to that imposed on the upstream half of the tube. These forces cause the flow tube to twist or bend. The forces applied to the flow tube switch direction with each half cycle.

The amount of twist or bend in the flow tube is directly proportional to the mass flow rate of material through the tube. The lateral velocities of both the upstream and downstream sides of the flow tube are measured. The time difference between the velocity detector signals appears as a phase shift and indicates the twist of the flow tube. The phase shift between the velocity signals indicates a mass flow rate. If no flow exists, then no twist is created in the flow tube, and the measured velocities are in phase. 
The density of the material in the flow tube is obtained from measuring the natural frequency of the system. The total mass of the tube and internal material is related to the natural frequency through the following relationship:

$$
\mathrm{m}_{\text {Total }}=\frac{\mathrm{k}}{\mathrm{f}^{2} 4 \pi^{2}}
$$

where $\mathrm{k}$ is the factory-determined spring constant and $\mathrm{f}$ is the natural frequency of oscillation. The density is obtained by subtracting the known mass of the tube and dividing by the internal volume of the flow tube.

An important fact to be noted about CFMs is that they are not designed to obtain an average value over the length of the flow tube. The fluid properties over the length of the flow tube are assumed constant. High-frequency transients such as slug flow or rapidly fluctuating slurry concentrations result in inaccurate measurements. Length scales associated with the nonhomogeneity of the fluid must be significantly greater than the length of the flow meter sensor tube.

It is the experience of PNNL's Fluids and Computational Engineering (FACE) Group that standard Micro Motion CMF sensors, transmitters, integrated flow meters, parts, and accessories are available either off-the-shelf or within a few weeks. Obviously, special orders may require significantly greater time to fill.

Coriolis meters are manufactured by Krohne, Endress \& Hauser, Foxboro, and Micro Motion. Micro Motion manufactures approximately $95 \%$ of the Coriolis meters currently in production. The majority of information pertaining to the application of Coriolis meters is associated with Micro Motion due to their overwhelming market share. Most of PNNL's experience within the DOE complex has been with Micro Motion meters, though there is some limited experience with Krohne meters (Reynolds et al. 1996; Enderlin et al. 1997, 2002; Powell et al. 1999). Straight tube Endress \& Hauser meters were installed in an experimental transfer line at PNNL's 338 Building fluids laboratory in late FY 2002. Based on these facts, various models of Micro Motion meters were evaluated to access the potential meter accuracy attainable.

Micro Motion's Elite series models are available for a flow range of approximately 500 to $2000 \mathrm{lb} / \mathrm{min}$ and include the CMF200 and CMF300, which are two- and three-inch models, respectively. The flow through both of these meters is routed through two channels with diameters considerably smaller than the nominal size of the meter. Based on vendor specifications, the accuracies attainable with these meters for mass flow rate are $0.16 \%$ and $0.18 \%$ of the reading for the CMF200 and CMF300, respectively. The specified uncertainty in density for both meters is $0.0005 \mathrm{~g} / \mathrm{mL}$.

The $\mathrm{T}$ series of Micro Motion meters consists of a single straight flow tube. The model T150 is the largest-diameter straight-tube configuration currently produced by Micro Motion. It has an internal diameter of 1.37 inches. The vendor-specified accuracy for a flow rate of $1000 \mathrm{lb} / \mathrm{min}$ is $0.2 \%$ of the reading; for the T150 the density accuracy is $0.002 \mathrm{~g} / \mathrm{mL}$. PNNL has no experience or process data obtained with a T-series meter. Product data sheets for Micro Motion CMF can be obtained via the Internet at www.emersonprocess.com/micromotion/products/indexspecsumm.html. 
Two-inch straight-tube Coriolis meters are manufactured by both Krohne (Model $800 \mathrm{G+}$ ) and Endress \& Hauser (Promass 40E). The Promass 40E has a range of 0 to $2600 \mathrm{lb} / \mathrm{min}$ and accuracy in mass flow rate of $0.5 \%$ of reading or higher depending on flow rate. The $800 \mathrm{G}+$ has a range of 33 to $3520 \mathrm{lb} / \mathrm{min}$ and a specified accuracy of $0.16 \%$ of reading or higher depending on flow rate.

Past discussions with vendor representatives have revealed that meter calibrations and accuracy specifications are based on testing with single-phase homogeneous standards. In addition to the following discussion concerning additional uncertainties involved with two-phase flow measurement, two attached appendixes describe Micro Motion Inc.'s factory calibration system and its traceability and uncertainties: Appendix A, "The Micro Motion TSM Calibration System," and Appendix B, "Traceability and Uncertainty Analysis for a Calibration Process for Flowmeters Using Coriolis Flowmeters as a Reference." While CMFs are used widely within industry to monitor multiphase and nonhomogeneous process flows, no vendor data or test results have been obtained.

Based on working experience and the review of process data for homogeneous slurries, the uncertainty in mass flow rate for the CMF200 is predicted to be $0.2 \%$ over the range of 1000 to $2000 \mathrm{lb} / \mathrm{min}$. The accuracy of the CMF300 is questioned due to the operating range of the meter. The process flow rate range specified for the waste transfers (e.g., AN-105 and AZ-102 waste transfers) was 1000 to $2000 \mathrm{lb} / \mathrm{min}$. The CMF300 operating range is 0 to $10000 \mathrm{lb} / \mathrm{min}$ and the CMF200, 0 to $3200 \mathrm{lb} / \mathrm{min}$. Applied to slurries, significant deviations from vendor specifications for accuracy have been observed at flow rates less than $20 \%$ of the meter range. Figure 3.1 plots meter accuracy and percent of meter full-scale flow rate. The vendor-specified accuracy is presented along with two plots that have been obtained from experimental testing at PNNL.

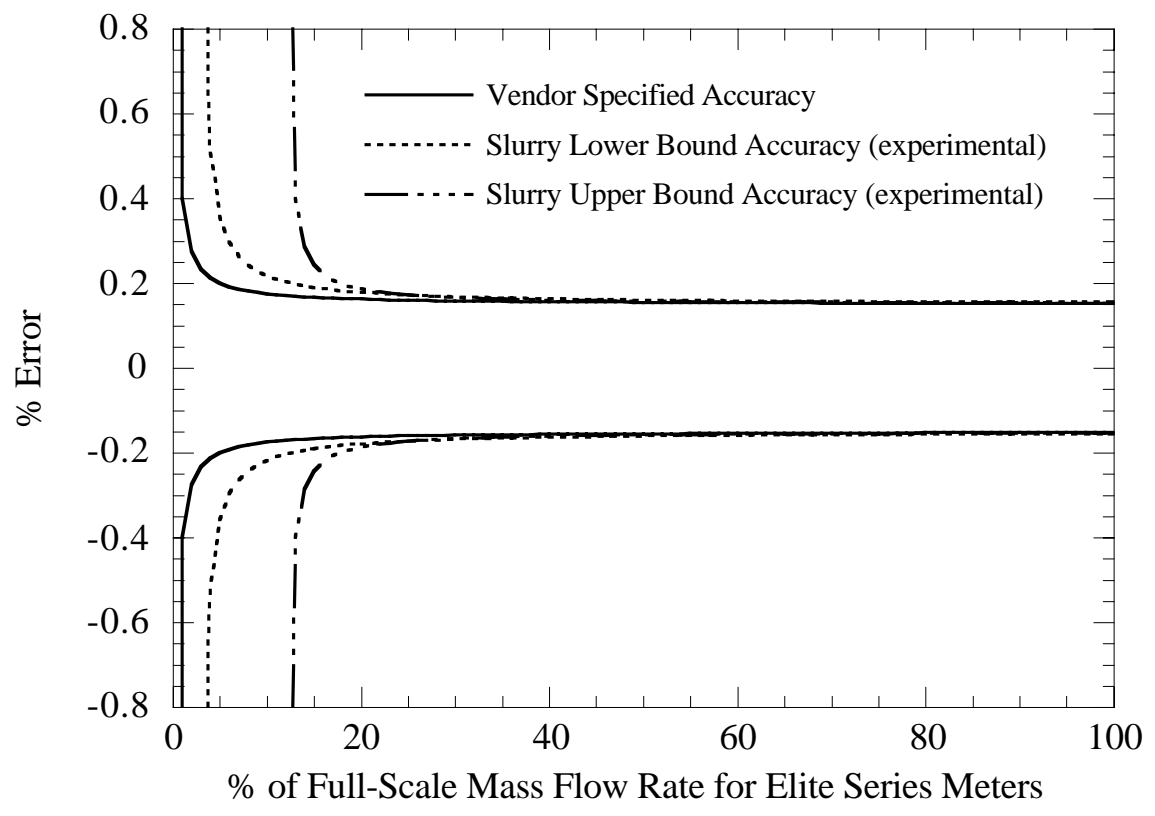

Figure 3.1. Percent Error as a Function of the Percent of Full-Scale Mass Flow Rate for Elite Series Micro Motion Flow Meters 
The two plots of experimental data shown in Figure 3.1 represent the upper and lower bounds of meter accuracy that have been observed during tests with suspended slurries. Testing has been conducted with slurries containing granular material such as zeolite and silica sand and cohesive material such as kaolin clay. The carrier fluids have consisted of water and mixtures of water, sugar, and salt. The specific gravities of the slurries have ranged from 1 to approximately 1.5. The bounding results presented are based on experimental work conducted at PNNL (Enderlin et al. 2002).

To provide an additional example of the uncertainty encountered by PNNL when measuring the mass flow rate of slurries with a CMF, an analysis of the percent error of a slurry transfer was performed. The data come from test 990916R1, performed in support of the Tanks Focus Areasponsored Scaled Flygt Mixer Testing-Phase D performed at PNNL (Enderlin et al. 2002). The slurry pumped was a mixture of water and $20 \times 50$ mesh zeolite (specific gravity 1.65). The meter in use was a 1-in Model DS100S128SU Micro Motion CMF.

The integral of the Micro Motion CMF measured mass flow rate was compared with the incremental changes in the weight of the test tank. Figure 3.2 plots the mass flow rate of the Zeolite slurry and percent error obtained throughout the transfer. The percent error fluctuated between $0.2 \%$ and $0.8 \%$.

- Mass rate $(\mathrm{Kg} / \mathrm{min})+\begin{gathered}\text { \% Error between tank weight and the } \\ \text { integral of the measured mass flow rate }\end{gathered}$

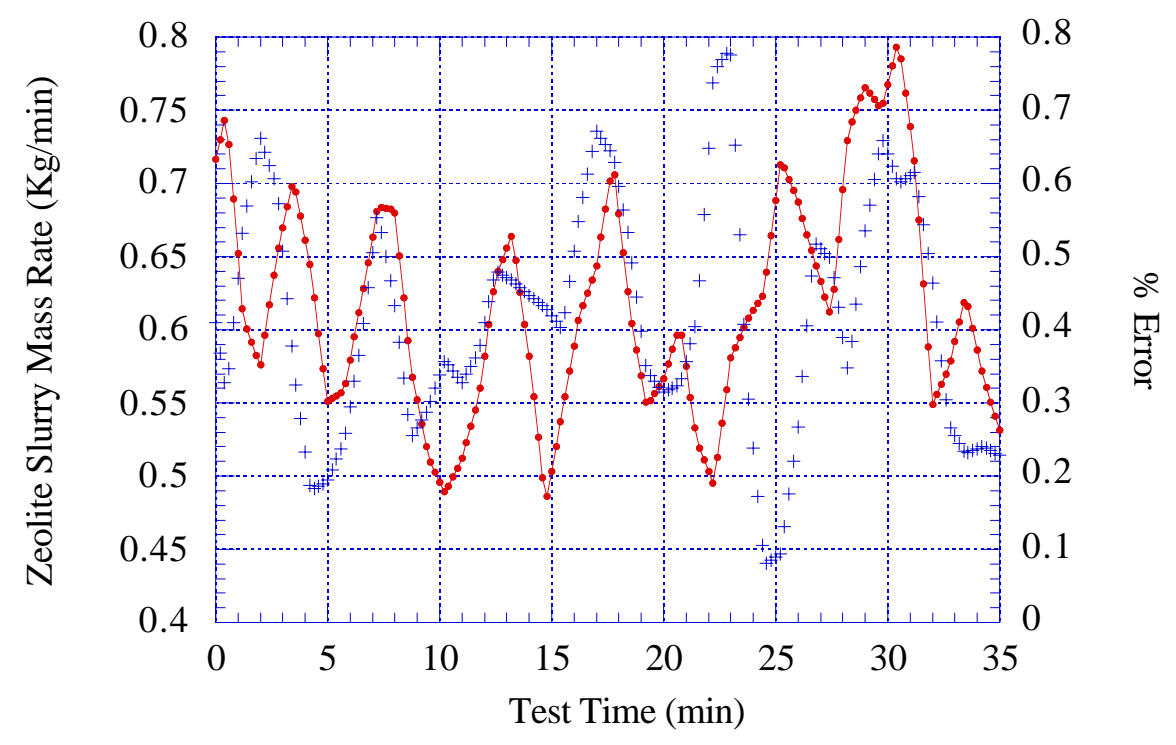

Figure 3.2. Zeolite Slurry Mass Flow Rate and Percent Error Between Test Tank Load Cell Readings and Integral of 1-in. Micro Motion CMF Meter Readings as a Function of Time. The data were obtained from a transfer operation during Phase-D Flygt Mixer Testing at the PNNL 1/4-Scale DST test facility. 
It is unclear how the observed deviations between the vendor-specified accuracy and the experimental results are related to various slurry properties such as particle concentration, particle settling velocity, size particle distribution, or homogeneity of the mixture. The accuracy of the instrumentation used to make comparative measurements also contributes to the deviations in accuracy observed between vendor specifications and the experimental results. No attempt has been made to analyze the results obtained because the obtained accuracies have always been within the desired limits.

An additional complication that has been observed in making comparisons to meter measurements is the introduction of gas coming out of solution within a pumped test loop. The gas tends to come out of solution much faster than it is absorbed. This effect has been observed in water tests, depending on the location of the CMF within the test loop. In a slurry, the microbubbles will adhere to the particulate, reducing the rate at which they dissolve back into solution. This complication is reduced when dealing with salt solutions of high ionic strength.

Testing of a CMF300 was performed at PNNL for Hanford Project W211 (Reynolds et al. 1996) at flow rates of approximately 1500 to $2200 \mathrm{lb} / \mathrm{min}$. The accuracy of the meter for mass flow rate was determined to be on the order of $8 \%$. Several items were considered to contribute to the high uncertainty, including the range of flow rates tested relative to the span of the meter. The results of the W211 testing were not considered in the development of Figure 3.1.

Coriolis meters are not affected by upstream piping configurations other than geometries that may allow gas to accumulate, which can result in periodic releases of bubbles, introducing noise in the measurements.

The Micro Motion meters are capable of measuring the flow rate in either direction through the meter. Programming options allow the meter to indicate positive flow in the forward, reverse, or both directions (i.e., all flow through the meter yields a positive value). No sacrifice in accuracy is incurred with the various options.

The recommended separate sensor and remote transmitter configuration obviously involves separate considerations for installation. For slurry media, manufacturers recommend that sensors be oriented in a vertical pipe run with flow upward. This orientation allows for media drainage when shut down and reduces the potential for particle accumulation.

Typical piping connections for the sensor unit include standard pipe flanges, tubing connections such as standard Swagelok, Swagelok VCO, or Sanitary Tri-Clamp. Pipe flange connections may require the most change-out time and complexity because of the time involved in manipulating the multitude of small parts, including bolts, nuts, and gaskets. Piping connections via sanitary tri-clamp fittings may be the simplest and least time-consuming method. Manipulation of the tri-clamp gaskets can be avoided by bonding them onto the sensor side of the tri-clamps.

If possible, mounting the sensor at the high point in the process line should be avoided to minimize gas accumulation in the meter. The installation should ensure that the CMF will not be subjected to mechanical vibration. Mounting multiple CMFs in proximity to one another should be reviewed to eliminate the potential for vibration crosstalk between sensors. 
The transmitter should be mounted to a structure at a convenient elevation and orientation for personnel to remove and reinstall covers and access electrical terminals and test points. Depending on the manufacturer and model, the sensor may be up to $1000 \mathrm{ft}$ (cable run) from the transmitter. The transmitter and sensor are interconnected by a special electric cable provided by the manufacturer.

The introduction of electrical noise to the CMF units should be minimized by:

- Powering the units using isolated circuits

- Following the manufacturer's recommendations for electrical connections and proper grounding

- Routing signal cables separately from sources of electrical noise

- Ensuring that neither the sensor nor the transmitter is installed close to generators of electrical noise, such as variable frequency drives.

Information on trouble shooting Micro Motion CMFs can be obtained from the Internet at http://www.emersonprocess.com/micromotion/library/index_transmitters.html.

Maintainability of CMFs is enhanced with separate sensors and transmitters. This configuration is also desirable in an application where the sensor will be handling high-level radioactive slurries. The transmitter electronics should be located some distance from the source of radiation. This allows transmitter reconfiguration and change-out with minimal radiation exposure to workers.

The only routine maintenance activity recommended by Micro Motion is periodic (flow) rezeroing of the transmitter to ensure that the sensor is full of media fluid and that no flow exists. Zeroing can be accomplished in two ways: 1) by removing a cover from the transmitter and pressing the zero button or 2) by connecting to the transmitter primary analog current output loop via a communication device (e.g., Hart) and zeroing electronically.

Most CMFs are available with some form of communication capability, either standard or optional, other than the industry standard $4 \sim 20 \mathrm{~mA}$ analog current loop signal outputs. Perhaps the most common implementation, and the one most familiar to PNNL's Face Group, is the Hart Protocol. The Hart Protocol is an industry standard promulgated by the Hart Foundation.

Hart Protocol provides a means of full-duplex communication with an instrumentation transmitter using existing 4 20mA loop wiring. This is accomplished by inserting Bell standard 202 frequency-shift-keyed digital data "on top" of the 4 20mA signal.

Micro Motion's transmitters insert Hart signal onto the primary variable 4 20mA loop for transmitters with more than one loop, or onto the single loop if only one is provided. A Hart Protocol signal can be inserted onto that loop by an external device to "talk to" the transmitter. The external device may consist of a handheld " 275 Hart Communicator" or a system such as Micro Motion's ProLink. The FACE Group prefers the ProLink because it uses a standard PC to display a full screen of data at one time, which greatly facilitates interpreting and editing transmitter configuration. 
ProLink consists of software and an RS-232 serial-to-Bell 202 signal converter. To use ProLink, one connects leads from the converter to test points on the transmitter, starts the software and, via a software command, connects to the transmitter. The ProLink software provides several screens, one dedicated to each available section of configuration, status reading, and testing.

The ProLink system interrogates the transmitter's memory and displays current configuration data on the PC's screen. Editable "calibration numbers" or configuration variables can be entered on-screen and sent to the transmitter, where they are stored in non-volatile ROM. For test purposes, commands can be sent to the transmitter to force it to send a desired current on a selected current loop. Among other capabilities, the transmitter can be zeroed via ProLink.

Some CMF transmitters use Hart Protocol to transmit sensed data. For example, most CMFs sense mass flow, density, and temperature (via an RTD in the sensor). Most CMF transmitters calculate volumetric flow from mass flow and density. The Micro Motion R-series transmitters, for example, only have one 4 20mA current loop. Thus, using the current loop alone, only one of these data values can be transmitted. However, the R-series employ the Hart Protocol to add the additional three data variables to the output by inserting them onto the current loop.

To access these data, another device is required to intercept or split them out. Such a device is the Moore Industries Hart Interface Module (HIM). The HIM can intercept up to four data variables from a current loop. The HIM is programmed by Moore-provided PC software to configure which data in the serial stream belongs to which variable. It splits these data out and creates up to four new 4 20mA current loops with those analog data on them. Thus, only one wire pair is required to carry four data streams, one of which is redundant.

Both the sensor and transmitter unit can be replaced individually. The remote location of the transmitter eliminates the potential for the transmitter to be contaminated. Transmitter replacement involves powering the system down; disconnecting power, sensor and output signal cables; unbolting and removing the unit; and following the reverse order of operations for installation of the new transmitter. Then the new transmitter must be configured for the particular sensor and operational requirements. This configuration is addressed in detail in the later discussion on calibration.

Sensor replacement in a nuclear environment poses unique problems not encountered with other process materials. The system design needs to account for sensor replacement after the unit has been contaminated and a calibration check performed on the installed replacement unit. The following paragraphs provide more detail on calibration for recommended capabilities.

As discussed in Appendix B, Micro Motion carefully calibrates CMFs at the factory. Micro Motion does not recommend routine field or factory calibrations. Usually, calibration facilities with uncertainties as low as the factory's calibration system are not available in the field.

It may be desirable to provide a means of functionality checking and rough calibration in a field installation. Such a capability could be built into a system using remote control valves to divert process water from a supply tank mounted on a weigh system through the flow sensor. The functional check could be performed during line-flushing operations or other normal process activities using process water, thus requiring no additional water (and no increase in waste 
volume). If the meter is set up correctly, the functional check can be performed with water flowing through the meter in either direction, allowing the current system pipe configuration to be used. A reasonable functionality check and rough field calibration can be made by monitoring and comparing the $\mathrm{CMF}$-indicated flow rate to the weight accumulation rate on the scale. If the installed orientation of the CMF allows the meter to be fully drained, a two-point check of the meter density can be made using air and process water.

\section{Improper Calibration}

PNNL's FACE group uses a variety of Micro Motion meters in various experimental setups. Each requires a different configuration of variables, units, ranges, and/or calibrations for various sensor/transmitter combinations. In this process, we have inadvertently misconfigured transmitters. While it is easy to configure with the ProLink system, it is just as easy to misconfigure with it. Errors in CMF configuration have been discovered through system reviews and shakedown tests. An appropriate QA system employed by individuals trained and skilled in system configuration will greatly minimize the probability of misconfiguration.

The configuration errors experienced by PNNL occur when reconfiguring and installing previously used units in a new experimental setup. For application in the Hanford waste transfer lines, a CMF system would be ordered with specific operating requirements. The desired variable ranges and outputs would be supplied to the manufacturer and the system configured by the vendor prior to calibration. For example, the customer may require that the primary variable analog output be scaled for 0 to $100 \mathrm{~kg} / \mathrm{min}$, the secondary variable analog output be scaled for 0 to $2 \mathrm{gm} / \mathrm{cc}$, the frequency output to be scaled to output 0 to $1000 \mathrm{~Hz}$ for $0^{\circ}$ to $100^{\circ} \mathrm{C}$, and the Hart output be configured to mirror these variables and ranges.

The manufacturer would first calibrate the sensor, determining that sensor's unique calibration numbers relating to mass flow, density, and temperature calibration. The sensor and transmitter are then interconnected and the transmitter software configured with the sensor's unique calibration numbers and the customer's required units, ranges, and outputs. The integrated system is then calibration-checked. Once configured, the configuration (including calibration) is maintained in nonvolatile ROM in the transmitter. Thus, the transmitter maintains the configuration throughout its lifetime, as it is disconnected from the sensor, shipped, stored, reconnected, and brought on-line in the field. As stated by Micro Motion (and most other manufacturers), the system does not usually need recalibration.

An exception may be if either the sensor or transmitter is replaced. In this case, the transmitter needs to be field-configured with the sensor's calibration numbers (provided by Micro Motion in calibration data and stamped onto metal plates welded onto the sensor) and the units, ranges, and outputs required for this application. This is relatively easily using a system such as Micro Motion's ProLink, which communicates with the transmitter via the Hart Protocol. The full screen view of calibration and configuration data and the ease of editing these facilitate reconfiguration.

It is the experience of PNNL's FACE Group that Micro Motion CMF sensors and transmitters are extremely reliable in dirty, wet lab environments where the units are continually being moved and reconfigured for a variety of experiments. Since 1994, when the group 
procured the first Micro Motion CMFs, only one malfunction has occurred. An RFT 9739 transmitter stopped working for an unknown reason. This transmitter was returned to the factory, repaired with the installation of updated electronics, and returned to PNNL in a few weeks.

Documentation of long-term reliability of a CMF in a demanding application is presented in Appendix C, the Micro Motion "Test Report, DS150S, S/N 138085," where it is reported that a DS150S sensor “...exceeded the customer's expectations for reliability and stability. In 9 years of operation, no maintenance on the meter was performed..." This sensor, connected to an RFT 9739 Field Mount Transmitter, monitored the flow and density of “...dirty and sour..." natural gas where previously used orifice plates needed continual maintenance and calibration.

It has been difficult to obtain specific, documented information from CMF vendors on the use of CMFs in radioactive environments. The sensor is the critical component that must be considered because the transmitters can be up to $1000 \mathrm{ft}$ from the sensor. Oak Ridge National Laboratory (ORNL) has tested a CMF that was handling radioactive slurries (Hylton et al. 1999). ORNL reported satisfactory performance of the tested Endress + Hauser Promass 63M CFM but unfortunately do not state the radiation intensity or dosage received by the flow meter.

The Promass 63M CFM has been discontinued and replaced with the Promass 83M, which is not currently certified for application in a radioactive environment. However, the upgrade from the Promass $63 \mathrm{M}$ to the $83 \mathrm{M}$ was in functionality within the transmitter. Therefore, there should be no difference in performance between the Promass 63M and 83M.

Appendix D (from Micro Motion) is a list of organizations and individuals known to have used Micro Motion CMFs in nuclear environments. PNNL contacted all of the organizations listed in Appendix D. Those organizations that replied were unable to provide additional details. Appendix E provides a list of the components and corresponding materials used in Micro Motions CMF100 and T100 sensors for evaluation of sensor in a radioactive environment.

A potential alternative exists to evaluate the radiation hardness of a CMF through operation in a controlled radioactive environment. PNNL possesses a "Gamma Pit" in the 3730 Building. It would be a straightforward process to conduct a radiation exposure test of a CMF system in this pit. Two CMF sensors would be connected in series in a test loop. One sensor, the unit being tested would be placed in a test tube in the pit, where it receives a known dose rate. Because of size limitations of the test tubes in the Gamma Pit, the sensor would be a specially configured version of a small, standard sensor. The reconfiguration would allow it to fit in the test tube but would not involve any change in construction materials or operation. A baseline sensor would be located outside the pit, receiving no radiation dose above normal background levels. A pump would be used to circulate water through the test loop, and both sensors would experience the same flow rate of the same fluid. Both transmitters would be located outside of the pit and configured to output mass flow, density and temperature. A PC-based data acquisition system monitors the transmitters' analog outputs. The output indications would be regularly monitored and checked for divergence. 


\subsubsection{Enraf Buoyancy Gauge}

The Enraf buoyancy gauge sits stationary on the waste or water surface. The instrument consists of a displacer suspended on a retractable/extendable wire connected to a force transducer. From Archimedes' principle, the displacer, when partially immersed in the waste surface, is buoyed up by a force equal to the weight of the displaced fluid.

The device operates by measuring the apparent weight of the displacer after partial immersion into the waste surface. This apparent weight is programmed into the device. In equilibrium conditions, the apparent weight of the displacer matches that measured by the force transducer. With changing waste level, the force transducer will experience a change in weight. The wire is then retracted/extended until the programmed apparent weight of the displacer is again measured by the force transducer. The change in wire length then gives the change from the reference level, allowing for determination of the new waste level. In this study, Yokogawa and Micro Motion flow meters and the Enraf buoyancy gauge were considered. Measurement errors associated with these devices are given in Table 3.1.

Table 3.1 Instrumentation Errors

\begin{tabular}{|l|l|l||}
\hline Device & Error & Distribution \\
\hline Yokogawa (AM400) & $\pm 1.0 \%$ & uniform \\
\hline Yokogawa (AM300D) & $\pm 0.5 \%$, water & uniform \\
\hline Micro Motion (R Series) & $\pm 0.5 \%$ & uniform \\
\hline Micro Motion & $\pm 0.2 \%$, waste & uniform \\
(Elite CMF200 or T150) & $\pm 0.25 \%$, water & normal \\
\hline Enraf & $\pm 0.00254 \mathrm{~m}$ & uniform \\
\hline
\end{tabular}

\subsection{Statistical Assessment Methodology}

A Monte Carlo simulation approach was used to investigate the error propagation associated with tank waste transfers. When adequate sampling is not achievable, Monte Carlo simulation studies can be employed to obtain good estimates of uncertainty.

Input distributions and their appropriate parameters are identified and determined, based on physical and engineering knowledge, to create scenarios that could possibly occur given every known possible combination of events. Each value used for the inputs is randomly sampled from an infinite population featuring the specified distribution and appropriate parameters. The typical distributions used for these inputs were uniform and normal (bell-shaped) distributions.

For each case, 2.5 million simulation runs were conducted. Sampling a large number of cases increases the chance that every possible combination of inputs is exhausted. The outputs represent all possible physical scenarios given the conditions constrained by the inputs. However, despite the number of runs conducted for each case, there is low probability that the extreme values for each parameter will be combined. Typically, therefore, the $100 \%$ interval results from these simulations are 0.5 to $10 \%$ less than conservative and highly unlikely pointestimation values, which combine all extreme parameter values. 


\subsection{Application Results and Evaluations}

The equations given in Section 2.2 were applied to the three specific transfer scenarios outlined in Section 3.1. The material balance error solely due to the instrumentation is discussed in Section 4.1. In this case, the waste properties and tank conditions are held constant. In Section 4.2, the waste properties and tank conditions are varied and the instrumentation errors altered to account for in-tank conditions to determine the total expected material balance error.

\subsection{Constant Waste Properties and Tank Conditions (instrumentation error)}

Material balance errors due solely to instrumentation error dictate a limit above which any material balance error identified could be attributed to effects other than the instrumentation. In other words, if the material balance error due to the instrumentation is larger than the error due to effects other than actual leakage or mis-routing, no definitive leak detection is available from the material balance. In this section, material balance errors are evaluated under constant waste properties and tank conditions.

\subsubsection{Summary of Input Data}

The constant waste properties and tank conditions for the three transfers are given in Table 4.1. Instrumentation errors are taken from Table 3.1. The waste data are taken or determined from Hedengren et al. (2000), Herting (1997), Schreiber (1995), and Bingham et al. (1999). Note that, in order to achieve the total transfer volume for Transfer 2 and 3, multiple transfers may be required for the vitrification plant tanks.

Table 4.1 Transfer Parameters

\begin{tabular}{|l|l|l|l|}
\hline \multicolumn{1}{|c|}{ Parameter (units) } & \multicolumn{1}{|c|}{$\begin{array}{c}\text { AN-105 } \\
\text { First } \\
\text { Transfer }\end{array}$} & $\begin{array}{c}\text { AN-105 } \\
\text { Second } \\
\text { Transfer }\end{array}$ & \multicolumn{1}{|c|}{ AZ-102 } \\
\hline Feed Waste Volume $(\mathrm{gal})$ & 584,000 & 673,000 & 150,000 \\
\hline Dilution Water Volume $(\mathrm{gal})$ & 327,400 & $\mathrm{NA}^{(\mathrm{a})}$ & NA \\
\hline Dilution Water Density $\left(\mathrm{kg} / \mathrm{m}^{3}\right)$ & 992 & $\mathrm{NA}$ & $\mathrm{NA}$ \\
\hline Feed Tank Diameter $(\mathrm{ft})$ & 75 & 75 & 75 \\
\hline Feed Waste Density $\left(\mathrm{kg} / \mathrm{m}^{3}\right)$ & 1430 & 1280 & 1147 \\
\hline Feed Tank Initial Height $(\mathrm{m})$ & 10.41 & 7.58 & 7.92 \\
\hline Feed Tank Final Height $(\mathrm{m})$ & 5.02 & 1.37 & 6.54 \\
\hline Receiver Tank Diameter $(\mathrm{ft})$ & 75 & 75 or 46 & 75 or 25 \\
\hline Receiver Tank Initial Height $(\mathrm{m})$ & 0.305 & NA & NA \\
\hline Receiver Tank Initial Density $\left(\mathrm{kg} / \mathrm{m}^{3}\right)$ & 1,000 & NA & NA \\
\hline Receiver Tank Final Height $(\mathrm{m})$ & 8.711 & 6.21, DST & 1.38, DST \\
\hline Receiver Tank Final Density $\left(\mathrm{kg} / \mathrm{m}^{3}\right)$ & 1269 & 1280 & 1147 \\
\hline (a) NA denotes not applicable. & \multicolumn{3}{|l}{} \\
\hline
\end{tabular}




\subsubsection{Waste Material Balance Results}

The material balance errors due to the instrumentation were evaluated using Equations (2.22), (2.23), and (2.24). As discussed in Section 3.3, a Monte-Carlo simulation approach was used for error propagation.

Three sets of measurements and three different transfer scenarios were considered, as presented in Sections 2.1 and 3.1. In addition, different flow meters and receiver tanks are considered. To facilitate the presentation and discussion of the results, these different cases are denoted in the following manner:

- Measurement Sets

A. Waste tank levels and diluent flow totalizer

B. Flow totalizer for diluted waste and receiver tank level

C. Waste and dilution tank levels

\section{- Transfer Scenarios}

1. AN-105 first transfer, 911,400 gallons

2. AN-105 second transfer, 673,000 gallons

3. AZ-102, 150,000 gallons

\section{- Flow Meters}

MM1. Micro Motion (R Series)

MM2. Micro Motion (Elite CMF 200 or T150)

Y1. Yokogawa (AM400)

Y2. Yokogawa (AM300D)

\section{- Receiver Tanks}

VPT. Vitrification plant tank No designation of a receiver tank indicates that it is a DST.

Therefore, case A/2/MM1/VPT denotes measurement set A, waste tank levels and diluent flow totalizer; Transfer scenario 2, AN-105 second transfer, 673,000 gallons; flow meter MM1, Micro Motion (R Series); and receiver tank VPT, the vitrification plant tank. Likewise, case A/2/MM1 would denote the same except the receiver tank would now be a DST.

The error results for Transfers 1,2, and 3 are presented in Table 4.2, 4.3, and 4.4, respectively. The $95 \%$ confidence interval and $100 \%$ interval (includes all outcomes given all input scenarios) for the largest material balance error are presented in the second and third columns. The percentage error of the $100 \%$ interval value compared with the total transfer is given in column 4 . Columns 5 and 6 indicate the confidence interval about the mean error. 
Table 4.2. Instrumentation Material Balance Error for Transfer 1

\begin{tabular}{|l|c|c|c|c|c|}
\hline \multirow{2}{*}{\multicolumn{1}{c|}{ Case }} & \multicolumn{2}{|c|}{ Error (gal) } & Error (\%) & \multicolumn{2}{c|}{ Error (gal) } \\
\cline { 2 - 6 } & $\mathbf{9 5 \%}$ CI & $\mathbf{1 0 0 \%}$ I & $\mathbf{1 0 0 \%}$ I & U 95\% Mean CI & L 95\% Mean CI \\
\hline $\mathrm{A} / 1 / \mathrm{MM} 1$ & -1700 & 2000 & -0.23 & 22 & -22 \\
\hline $\mathrm{A} / 1 / \mathrm{MM} 2$ & 800 & 1300 & 0.14 & 11 & -10 \\
\hline $\mathrm{A} / 1 / \mathrm{Y} 1$ & 2500 & 3300 & -0.36 & 42 & -40 \\
\hline $\mathrm{A} / 1 / \mathrm{Y} 2$ & -1700 & 2000 & -0.23 & 22 & -22 \\
\hline $\mathrm{B} / 1 / \mathrm{MM} 1$ & 4600 & 5000 & -0.54 & 74 & -72 \\
\hline $\mathrm{B} / 1 / \mathrm{MM} 2$ & 1800 & 2200 & -0.24 & 29 & -31 \\
\hline $\mathrm{B} / 1 / \mathrm{Y} 1$ & 8700 & 9400 & -1.05 & 149 & -141 \\
\hline $\mathrm{C} / 1$ & -600 & -1000 & -0.11 & 9 & -9 \\
\hline
\end{tabular}

Table 4.3. Instrumentation Material Balance Error for Transfer 2

\begin{tabular}{|l|c|c|c|c|c|}
\hline \multirow{2}{*}{ Case } & \multicolumn{2}{|c|}{ Error (gal) } & Error (\%) & \multicolumn{2}{c|}{ Error (gal) } \\
\cline { 2 - 6 } & $\mathbf{9 5 \%}$ CI & $\mathbf{1 0 0 \%}$ I & $\mathbf{1 0 0 \%}$ I & U 95\% Mean CI & L 95\% Mean CI \\
\hline $\mathrm{A} / 2$ & 500 & 800 & 0.11 & 7 & -8 \\
\hline $\mathrm{A} / 2 / \mathrm{VPT}$ & -1300 & 1900 & -0.28 & 19 & -20 \\
\hline $\mathrm{B} / 2 / \mathrm{MM} 1$ & 3500 & 3600 & -0.54 & 54 & -54 \\
\hline $\mathrm{B} / 2 / \mathrm{MM} 2$ & 1300 & 1600 & -0.24 & 22 & -22 \\
\hline $\mathrm{B} / 2 / \mathrm{Y} 1$ & 6400 & 7000 & -1.04 & 107 & -108 \\
\hline $\mathrm{B} / 2 / \mathrm{MM} 2 / \mathrm{VPT}$ & 1400 & 1600 & -0.24 & 23 & -21 \\
\hline $\mathrm{C} / 2$ & 500 & -800 & -0.12 & 8 & -7 \\
\hline $\mathrm{C} / 2 / \mathrm{VPT}$ & -1300 & 1900 & -0.28 & 19 & -20 \\
\hline
\end{tabular}

Table 4.4. Instrumentation Material Balance Error for Transfer 3

\begin{tabular}{|l|c|c|c|c|c||}
\hline \multirow{2}{*}{ Case } & \multicolumn{2}{|c|}{ Error (gal) } & Error (\%) & \multicolumn{2}{c||}{ Error (gal) } \\
\cline { 2 - 6 } & $\mathbf{9 5 \%}$ CI & $\mathbf{1 0 0 \%}$ I & $\mathbf{1 0 0 \%}$ I & U 95\% Mean CI & L 95\% Mean CI \\
\hline A/3 & 500 & -800 & -0.52 & 8 & -7 \\
\hline A/3/VPT & -900 & 1100 & -0.75 & 13 & -12 \\
\hline B/3/MM1 & 900 & 1000 & -0.68 & 12 & -13 \\
\hline B/3/MM2 & -400 & -600 & -0.38 & 6 & -7 \\
\hline B/3/Y1 & 1500 & 1700 & -1.18 & 25 & -23 \\
\hline B/3/MM2/VPT & 300 & 400 & -0.24 & 5 & -5 \\
\hline C/3 & 500 & 800 & 0.52 & 8 & -8 \\
\hline C/3/VPT & 900 & 1100 & -0.75 & 13 & -12 \\
\hline
\end{tabular}

\subsubsection{Evaluation}

The material balance errors due to the instrumentation are relatively small, typically accounting for less than $1 \%$ of the total transfer. For transfer into another DST, the 100\% interval error for Transfer 1 ranged from 1,000 to 2,200 gallons, for Transfer 2 from 800 to 1,600 gallons, and for Transfer 3 from 600 to 800 gallons. The results for transfer to the vitrification plant tanks depended on the diameter of the tank and the number of transfers required to achieve 
the same total transfer volume. For Transfer 2, the material balance error ranged from 1,600 to 1,900 gallons, and for Transfer 3 from 400 to 1,100 gallons. The upper and lower 95\% confidence intervals about the mean show that, for each transfer, the main error was approximately zero. The material balance error due to the instrumentation is small enough that the error due to effects other than actual leakage or misrouting is identifiable.

In general, height measurements alone produced lower material balance errors. However, as discussed in detail in Section 4.2.1, the variable waste properties and tank conditions that are to be expected during the transfer of saturated wastes make the height measurements subject to significant amounts of error. In instances where the feed or receiver tank has constant waste properties and tank conditions, the instrumentation material balance error would apply. This situation could occur in transfer scenarios 2 and 3 with measurement set B. For these cases, there are variable waste properties and tank conditions in the feed tank and the mass flow meter in the pipeline would provide accurate density measurements of the waste being transferred into the receiver tank. Therefore, the instrumentation material balance error would apply for the receiver tank.

\subsection{Variable Waste Properties and Tank Conditions}

It has been established in Section 4.1 that the expected transfer instrumentation will provide sufficiently accurate results to be able to assess the material balance error due to variable waste properties and tank conditions. The factors affecting the waste properties, tank conditions, and measurements are considered in detail and are applied to the material balance equations. These results are presented in Sections 4.2.6 through 4.2.8.

\subsubsection{Factors Affecting Accurate Measurements of the Waste Height}

Height measurements in the feed, receiver, and water tank may be affected by the following factors:

- Density changes due to chemical reactions

- Density changes due to gas generation, retention, and release

- Density changes due to temperature change

- Heterogeneous waste distribution

- Existence of crust and its potential dissolution

- Changes of tank cross-sectional area (e.g., due to crust hanging on the tank wall)

- Waste surface disturbance due to mixer and transfer pump operations and waste distribution.

Density changes have the potential to alter the apparent volume of the waste by as much as 7\% (based on numerous dilution studies performed on Hanford waste, documented gas generation, retention and release behaviors, and known chemical solubility data). Factors such as waste surface irregularities can also cause uncertainties in the height measurement of up to $0.2 \mathrm{~m}$ (Hedengren et al. 2000). Photographs of the waste surfaces in many of the Hanford tanks show considerable irregularity. Double-shell tank 241-SY-101 (SY-101) has two Enraf devices 
deployed in it, and, prior to the transfer activities, they differed approximately $0.5 \mathrm{~m}$ in waste height indication (about 54,000 gallons of waste) due to their different locations on the waste surface (Kirch et al. 2000).

The 1999-2000 transfers of SY-101 diluted slurry to DST 241-SY-102 (SY-102) provides an illustrative example of the difficulties encountered using height measurements for a material balance. Prior to the transfer activities, SY-101 had the most concentrated waste of any of the DSTs and had developed a crust layer approximately $3 \mathrm{~m}$ thick. Approximately 15,000 scf of gas was retained in the tank (Rassat et al. 2000). SY-102 continues to serve as a receiver tank for process water and salt-well pumped liquid from other 200 West Area tanks. It contained approximately $7.4 \mathrm{~m}$ of dilute waste prior to the first transfer from SY-101. Waste height measurements in both SY-101 and SY-102 were recorded during the transfers. A Yokogawa flow meter was installed in the transfer line. An overview of the tank histories and detailed presentation and discussion of the transfer data may be found in Mahoney et al. (2000).

The potential existed for many of the previously listed factors to affect the height measurement in SY-101, but the potential was much less in SY-102. The flow meter total flow is compared in Table 4.5 with that determined from the level measurements in each of the tanks for each of the three transfers conducted. The discrepancy between the level and flow meter measurements for the first transfer is about 23\% for SY-101. As the gas inventory in SY-101 was reduced and the subsequent back-dilutions dissolved the crust layer, the apparent error from the level measurement in SY-101 decreased significantly.

Table 4.5. SY-101 Transfer Volume Comparison

\begin{tabular}{|c|c|c|c|c|c|}
\hline \multirow[b]{2}{*}{ Transfer } & \multicolumn{3}{|c|}{ Transfer Volume } & \multirow[b]{2}{*}{$\begin{array}{c}\text { SY-101 SL } \\
\text { \% Error } \\
\text { (to flow } \\
\text { meter) }\end{array}$} & \multirow[b]{2}{*}{$\begin{array}{c}\text { SY-102 SL } \\
\text { \% Error } \\
\text { (to flow } \\
\text { meter) }\end{array}$} \\
\hline & $\begin{array}{c}\text { Yokogawa } \\
\text { Flow Meter } \\
\text { (gal) }\end{array}$ & $\begin{array}{c}\text { SY-101 } \\
\text { Surface } \\
\text { Level }^{(a)} \\
\text { Enraf 1A } \\
\quad \text { (gal) }\end{array}$ & \begin{tabular}{|c|} 
SY-102 \\
Surface \\
Level Enraf \\
(gal)
\end{tabular} & & \\
\hline 1 & 170050 & 209304 & 169976 & 23.08 & -0.04 \\
\hline 2 & 432900 & 483326 & 418662 & 11.65 & -3.29 \\
\hline 3 & 383240 & 395606 & 387432 & 3.23 & 1.09 \\
\hline
\end{tabular}

Gas release alone from SY-101 could account for a bulk density change of 5\%, amounting to almost $0.5 \mathrm{~m}$ of waste height. There is also photographic evidence of the retention of a 1-mthick crust ring on the tank wall in SY-101 during the first and second transfers. Figure 4.1 shows the waste surface in SY-101 during the first transfer. The center of the waste surface has lowered due to the transfer, but a ledge or shelf of material can be seen close to the tank wall that has retained its original position. During the second transfer and back-dilution, this retention on the wall was even more apparent. Figure 4.2 shows the waste material on the wall during the back-dilution following the second transfer. The waste material covering the wall area on the left side of the picture had recently sloughed off, leaving the waste material seen on the right side. For a 1,000,000-gallon transfer, wall retention of crust material of this magnitude could account for a $0.2 \mathrm{~m}$ change in the waste height ( 22,000 gallons). 


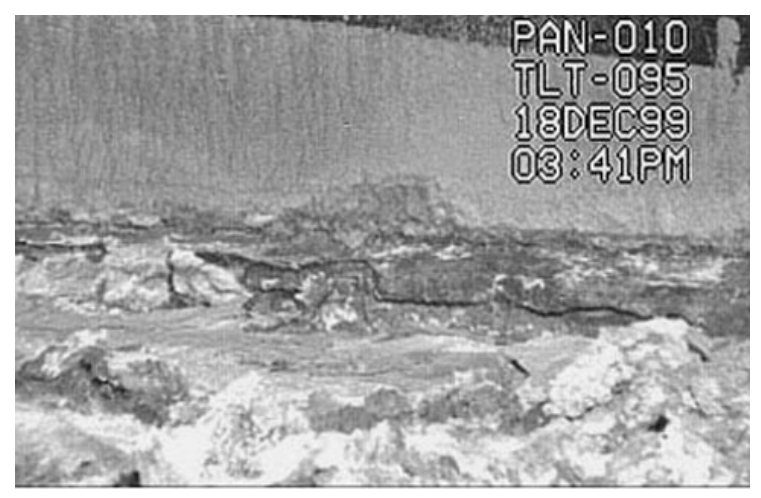

Figure 4.1 Tank Wall Waste Retention During Transfer 1

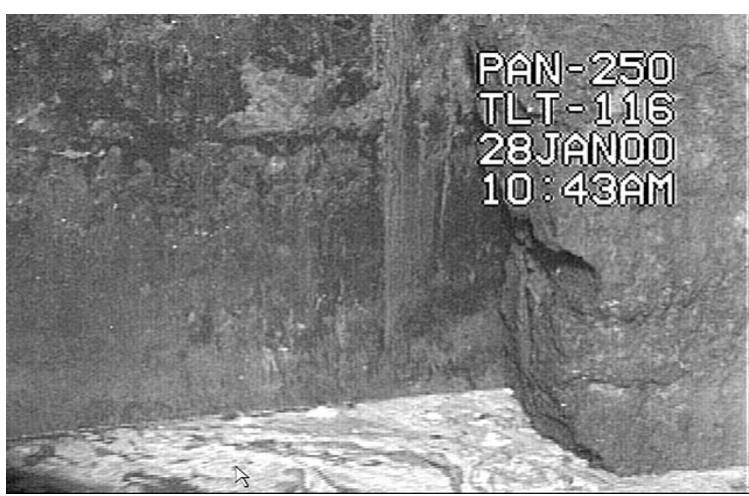

Figure 4.2 Tank Wall Waste Retention After Transfer 2

The effects of waste surface irregularities on the height measurement were also demonstrated during the SY-101 transfer. During the back-dilution after the second transfer, the bulk of the crust layer was dissolved. The Enraf in riser $1 \mathrm{~A}$ was observed to be significantly affected by this process. The Enraf displacer can be seen on the solid waste surface in the upper left photo in Figure 4.3. The displacer is approximately 4 in. from top to bottom. As the back-dilution progressed, the solids subsided into the liquid below, burying the displacer (lower left photo). The displacer returned to a level position on the liquid surface approximately 10 hours later. The change in position of the Enraf displacer over the 16 hour period due to changing waste surface can account for approximately $0.4 \mathrm{~m}$ of apparent waste height change ( 40,000 gallons).
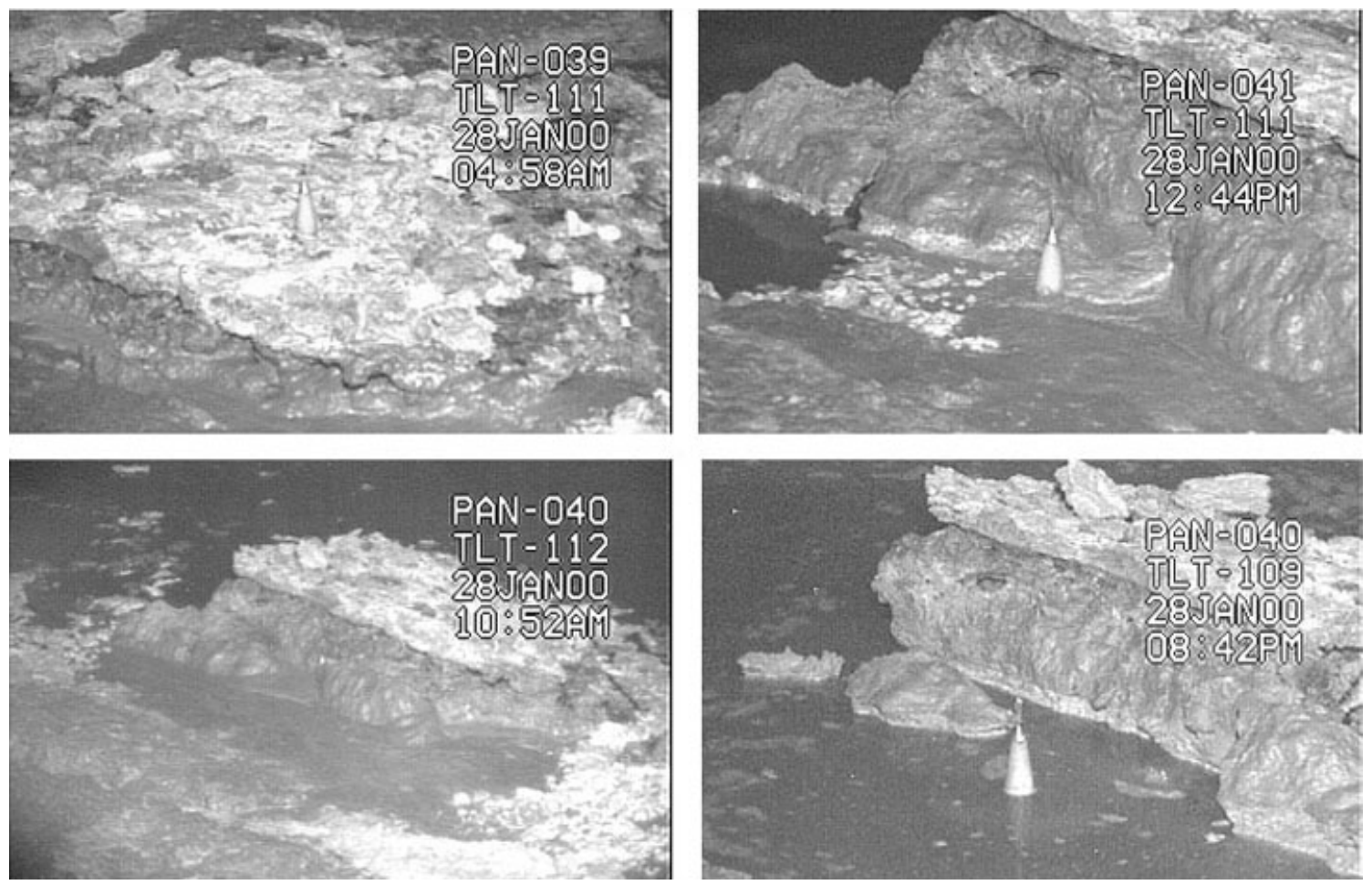

Figure 4.3. Waste Surface Irregularity Effect on Height Measurements 


\subsubsection{Determination of Height Measurement Accuracy}

As discussed in Section 4.2.1, numerous factors can significantly affect the height measurement. In this section, the methods used to determine the accuracy of the waste height for this study are presented.

The most significant factor affecting waste height measurement, as was demonstrated in SY-101, is the existence of a crust layer. Surface irregularities and apparent change of the tank cross-sectional area due to retention of waste material on the walls were visually observed during the transfers.

For the three transfer scenarios we considered, only AN-105 during the first transfer is expected to have a crust layer. The uncertainty in the height measurement of the feed tank for this transfer is therefore taken to be $0.2 \mathrm{~m}$ (Hedengren et al. 2000). The effect of possible material retention on the walls is applied by comparison to that seen in SY-101. The expected material retention is distributed over the tank wall exposed by the transfer, and the resulting difference in the cross-sectional area is applied as the uncertainty to the area. For those transfers with no crust layer in the feed tank, or a liquid surface or empty receiver tank, the uncertainty of the waste height is taken to be that of the Enraf instrument, or $0.00254 \mathrm{~m}$.

The effect of possible mixer pump operation during the transfer is also considered. During the transfer from AZ-102, a mixer pump is expected to be employed. There is some evidence from the recent mixing operations in AZ-101 that the action of the mixer pump can disturb the waste surface. Additionally, numerical simulations of tank-mixing processes have been conducted which provide detailed shear and velocity measurements at the waste surface. It is estimated that these effects can introduce an uncertainty of about $2 \mathrm{~cm}$ into the waste height measurement.

\subsubsection{Factors Affecting Accurate Measurements of the Waste Flow Rate}

Waste flow rates are also subject to uncertainty. Factors that may affect measurement of the flow rate include

- Flow rate out of optimum range of instrument

- Gas accumulation in pipeline due to pipe routing or component configurations

- Deviation from vendor-specified pipe configurations (volumetric flow meter)

- Installation in proximity to power sources

- Orientation of meter relative to phase/s (gas, liquid, and solid) of material flow

- Excessive vibration from external sources (mass flow meter)

- High concentrations of metallic particulate (mass flow meter)

- Heterogeneous waste stream (high-frequency transients in density, mass flow meter)

- Heterogeneous waste stream (vertical distribution, volumetric flow meter)

- Density changes due to temperature changes (volumetric flow meter). 
The majority of these factors can be controlled by choosing the correct instrument for the task and installing it correctly. Therefore, measurement of the flow rate will provide better data for a material balance than the waste height measurements. As with the height measurements however, accurate data for the density of the waste is a critical parameter.

\subsubsection{Methods to Determine Waste Density}

To achieve accuracy in a waste transfer material balance, the waste must be well classified. As discussed in the previous sections, the waste density is a crucial parameter. In this section, methods that may be used to determine the waste density due to chemical reactions and gas content are discussed.

\subsubsection{Chemical Reaction Effects on Waste Density}

Waste density in the Hanford tanks is affected by

- Dissolution of solids

- Precipitation of solids

- Mixture of a waste solution with water

- Mixture of different waste solutions

Five methods have been identified to determine waste density under these conditions. They are presented in order from the most preferable to the least accurate.

1. Densitometer: A mass flow meter provides density information. In-process density measurements are inherently the most accurate.

2. Laboratory Experiments: Although laboratory experiments cannot duplicate in-tank processes exactly, they can provide quantitative data for chemical reactions, solid and liquid phase concentrations, and the solution density from tank waste or simulants (e.g., Herting 1997, 1998). These data can be extrapolated to tank or transfer conditions.

3. Chemical Modeling: The computer modeling can be coupled with the first two methods to provide a means of checking, adding additional information, or, in instances of no actual tank data, used alone to provide detailed information on the waste chemistry under varying conditions (Onishi et al. 2000).

4. Empirical Formula from Hanford Wastes: Extrapolates solution density under varying conditions based on empirical relations from data encompassing all or some of the Hanford tanks. This can be coupled with methods 2 and 3 to complete data sets.

5. Assign a Value by Comparison: Using the known data of a particular waste, the solution density is assigned based on comparisons of that data to other Hanford waste or solution chemistry data. This is a judgment-based method, so it is therefore subject to large uncertainties. 


\subsubsection{Gas Content Effects Waste Density}

Gas is generated, retained, and released in varying quantities by the waste in the Hanford tanks. Extensive studies have been conducted to explain and quantify the gas content (e.g., Mahoney et al. 1999). The effect of this gas content on the waste density (and therefore waste volume) during a transfer scenario can be determined by:

1. Determining the gas content of the transferred waste. This may be achieved by direct in situ measurements, tank dome space gas specie concentrations, laboratory experiments with tank waste or simulants, or comparison with similar wastes.

2. Determining the effect of transfer activities on gas content. Again, in-tank data from prior transfers or laboratory experiments can be used. The effect may also be bounded by assuming that all the gas is either retained or released by the transfer.

The preparation of the tank for the transfer must be considered as well. A well-mixed tank will have released most of its retained gas; supernatant liquid with no solids content will only have transient gas in it.

\subsubsection{Determination of Waste Density}

Methods discussed in Subsections 4.2.4.1 and 4.2.4.2 were used in this study to determine the waste density. The methods employed for each transfer are discussed here.

Density data have been documented from core and grab samples describing the current waste properties in AN-105 and AZ-102. These data sources include Hedengren et al. (2000), Bingham et al. (1999), Herting (1997), and Schreiber (1995). The supernatant removed from AN-105 during the first transfer is diluted at $56 \%$ by volume with water in-line. No dilution data exist for AN-105 supernatant liquid and water. The resultant mixture density is therefore determined based on a volumetric approach. Comparison of supernatant dilution data from DST 241AN-104 (AN-104; waste similar to AN-105), however, shows that volume is not conserved. The uncertainty of the result of the volumetric average density in AN-105 is therefore taken to be that shown in the AN-104 studies (Herting 1998).

This dilution process is also considered with the chemical model GMIN (Felmy 1995). The initial aqueous species in the supernatant liquid in AN-105 are presented in Figure 4.4. Note that the measurement accounted for total sodium, total nitrate, etc., while the chemical model accounted for sodium, sodium nitrate, etc. The summations show good agreement.

Upon dilution with water at $56 \%$ by volume, there is indication that gibbsite may precipitate (see Figure 4.5$)$. However, the quantity ( $0.6 \%$ by volume) is minimal and has minimal effect on the waste density. The effect is less than that seen in AN-104 (Herting 1998) or, in regard to its effect on the apparent volume of waste in the tank, less than the $0.00254 \mathrm{~m}$ of uncertainty in the Enraf. Furthermore, the kinetic rate to form gibbsite is expected to be much greater than the duration of the waste transfer. The possible effect is therefore ignored in this case. This example does, however, serve as a poignant illustration of the value of considering chemical modeling to identify and avoid potential complications. 


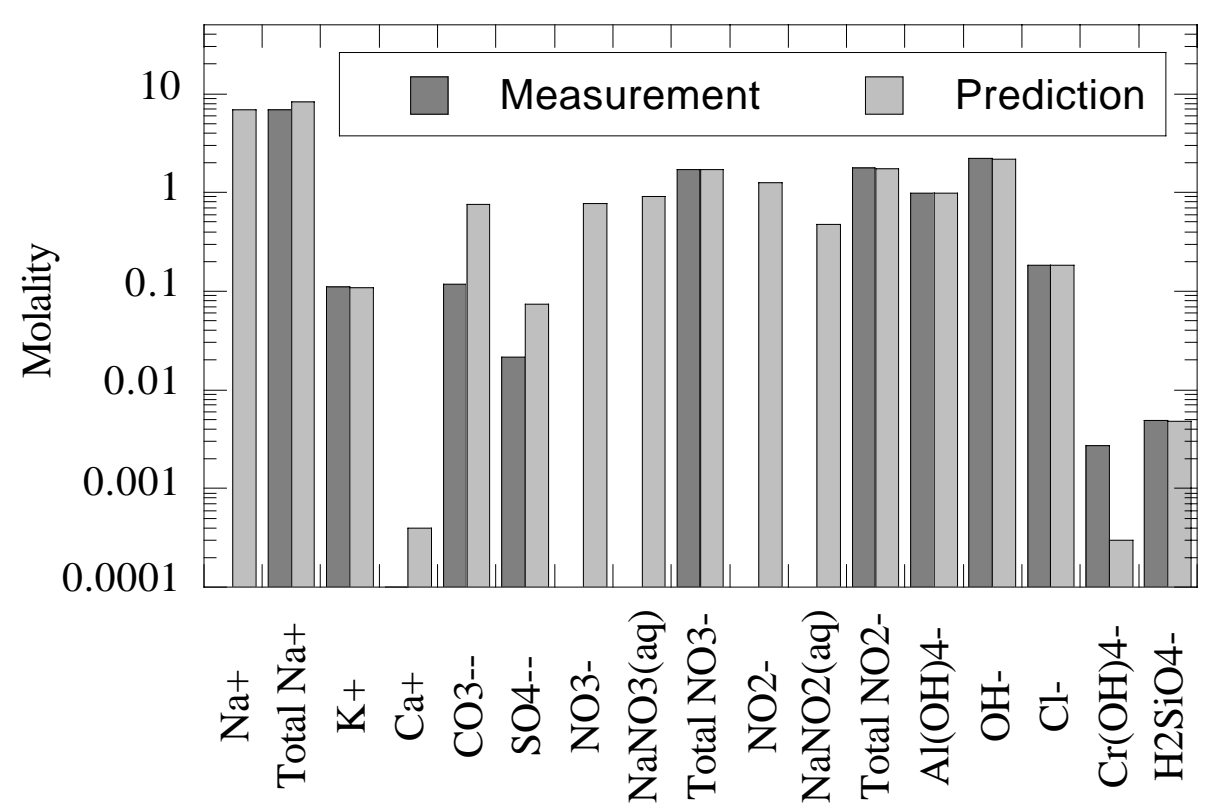

Figure 4.4. Measured and Predicted Supernatant Liquid Chemical Concentrations for AN-105

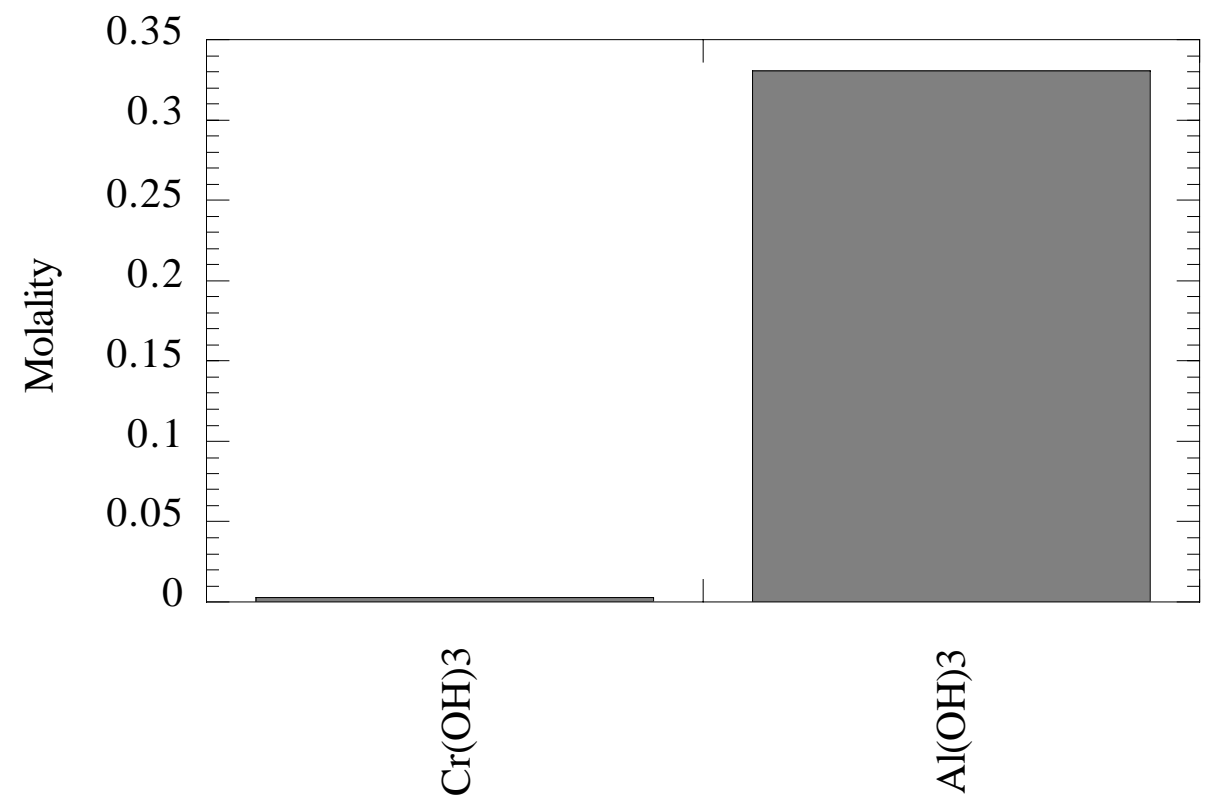

Figure 4.5. Predicted Dry Solid Concentration for AN-105 Supernatant Liquid Diluted at $56 \%$ by Volume with Water 
The supernatant liquid in AN-105 also contains gas at $2 \times 10^{-5} \%\left( \pm 1 \times 10^{-5} \%\right)$ by volume (Mahoney et al. 1999). It was assumed that this gas is all released, partially released, or completely retained after the transfer. This effect is included in the uncertainty of the density of the mixture.

Herting (1997) diluted AN-105 settled solids at $80 \%$ by volume with water. We therefore have the necessary data point to describe the density of the supernatant liquid in AN-105 prior to the second transfer. Unfortunately, only a single liquid density measurement is reported. Herting's dilution study in AN-104 (Herting 1998) was again used to supplement the available data. As the liquid density measurement techniques can be assumed to be the same in each study, the standard deviation of the measurements in AN-104 (settled solids diluted at $80 \%$ by volume with water) were used as the expected uncertainty in the reported AN-105 density.

For transfer of slurry from AZ-102, the density and solids content were determined by volumetrically mixing the supernatant and settled solids. Initial waste properties were taken from Bingham et al. (1999) and Schreiber (1995). The uncertainty of the slurry density was computed by propagation of the initial condition uncertainties.

\subsubsection{Summary of Input Data}

The variable waste properties and tank conditions for the three transfers are given in Tables 4.6, 4.7 and 4.8. The waste data are taken or determined from Hedengren et al. (2000),

Table 4.6. AN-105 first Transfer Parameters

\begin{tabular}{|c|c|c|c|}
\hline Parameter (units) & $\begin{array}{c}\text { AN-105 } \\
\text { First Transfer }\end{array}$ & Uncertainty & Distribution \\
\hline Water Mass Flow Rate (kg/min) & 188.48 & $\pm 0.25 \%$ & normal \\
\hline Dilution Water Density $\left(\mathrm{kg} / \mathrm{m}^{3}\right)$ & 992 & $\pm 0.02 \%$ & uniform \\
\hline Water Flow Rate $\left(\mathrm{m}^{3} / \mathrm{min}\right)$ & 0.19 & $\pm 0.5 \%$ & uniform \\
\hline Water Tank Initial Height (m) & 7 & \pm 0.00254 & uniform \\
\hline Water Tank Final Height $(\mathrm{m})$ & 0.213 & \pm 0.00254 & uniform \\
\hline Water Tank Area $\left(\mathrm{m}^{2}\right)$ & 182.4 & $\mathrm{NA}^{(\mathrm{a})}$ & NA \\
\hline Feed Tank Area $\left(\mathrm{m}^{2}\right)$ & 410.433 & $\begin{array}{l}\min -0.79 \\
\text { max at value }^{(b)}\end{array}$ & $\begin{array}{l}\text { triangular } \\
\text { max at value }^{(b)}\end{array}$ \\
\hline Feed Waste Density $\left(\mathrm{kg} / \mathrm{m}^{3}\right)$ & 1430 & \pm 30 & normal \\
\hline Feed Tank Initial Height (m) & 10.41 & \pm 0.2 & uniform \\
\hline Feed Tank Final Height $(\mathrm{m})$ & 5.02 & \pm 0.2 & uniform \\
\hline Feed and Water Mass Flow Rate (kg/min) & 669.39 & $\pm 0.2 \%$ & uniform \\
\hline Transfer Time (min) & 6509 & \pm 1 & uniform \\
\hline Receiver Tank Area $\left(\mathrm{m}^{2}\right)$ & 410.433 & NA & NA \\
\hline Receiver Tank Initial Height (m) & 0.305 & \pm 0.00254 & uniform \\
\hline Receiver Tank Initial Density $\left(\mathrm{kg} / \mathrm{m}^{3}\right)$ & 1,000 & $\pm 0.09 \%$ & uniform \\
\hline Receiver Tank Final Height (m) & 8.711 & \pm 0.00254 & uniform \\
\hline Receiver Tank Final Density $\left(\mathrm{kg} / \mathrm{m}^{3}\right)$ & 1269 & $\begin{array}{l}\min -1.21 \% \\
\text { mean }-0.54 \% \\
\text { max at value }\end{array}$ & skewed normal \\
\hline
\end{tabular}


Table 4.7. AN-105 Second Transfer Parameters

\begin{tabular}{||l|l|l|l||}
\hline \multicolumn{1}{|c|}{ Parameter (units) } & \multicolumn{1}{|c||}{$\begin{array}{c}\text { AN-105 } \\
\text { Second } \\
\text { Transfer }\end{array}$} & \multicolumn{1}{|c||}{ Uncertainty } & \multicolumn{1}{|c||}{ Distribution } \\
\hline Feed Tank Area $\left(\mathrm{m}^{2}\right)$ & 410.433 & $\mathrm{NA}^{(\mathrm{a})}$ & NA \\
\hline Feed Waste Density $\left(\mathrm{kg} / \mathrm{m}^{3}\right)$ & 1280 & $\mathrm{SD}=14.14$ & normal \\
\hline Feed Tank Initial Height $(\mathrm{m})$ & 7.58 & \pm 0.00254 & uniform \\
\hline Feed Tank Final Height $(\mathrm{m})$ & 1.37 & \pm 0.00254 & uniform \\
\hline Feed Mass Flow Rate $(\mathrm{kg} / \mathrm{min})$ & 678.4 & $\pm 0.2 \%$ & uniform \\
\hline Transfer Time $(\mathrm{min})$ & 4809 & \pm 1 & uniform \\
\hline Receiver Tank Area $\left(\mathrm{m}^{2}\right)$ & 410.433 & $\mathrm{NA}$ & NA \\
\hline Receiver Tank Initial Height $(\mathrm{m})$ & 0.0 & $\mathrm{NA}$ & NA \\
\hline Receiver Tank Initial Density $\left(\mathrm{kg} / \mathrm{m}^{3}\right)$ & 0.0 & $\mathrm{NA}$ & NA \\
\hline Receiver Tank Final Height $(\mathrm{m})$ & 6.21 & \pm 0.00254 & uniform \\
\hline Receiver Tank Final Density $\left(\mathrm{kg} / \mathrm{m}^{3}\right)$ & 1280 & SD $=14.14$ & normal \\
\hline (a) NA denotes not applicable. & \multicolumn{2}{|l}{} \\
\hline
\end{tabular}

Table 4.8. AZ-102 Transfer Parameters

\begin{tabular}{||l|l|l|l||}
\hline Parameter $($ units) & AZ-102 & Uncertainty & Distribution \\
\hline Feed Tank Area $\left(\mathrm{m}^{2}\right)$ & 410.433 & $\mathrm{NA}^{(\mathrm{a})}$ & NA \\
\hline Feed Waste Density $\left(\mathrm{kg} / \mathrm{m}^{3}\right)$ & 1147 & \pm 47 & normal \\
\hline Feed Tank Initial Height $(\mathrm{m})$ & 7.92 & $\begin{array}{l} \pm 0.00254 \\
\pm 0.02^{(b)} \\
\pm 0.00254 \\
\pm 0.02^{(b)}\end{array}$ & uniform \\
\hline Feed Tank Final Height $(\mathrm{m})$ & 6.54 & $\pm 0.2 \%$ & uniform \\
\hline Feed Mass Flow Rate $(\mathrm{kg} / \mathrm{min})$ & 607.91 & \pm 1 & uniform \\
\hline Transfer Time (min) & 1069 & NA & NA \\
\hline Receiver Tank Area $\left(\mathrm{m}^{2}\right)$ & 410.433 & NA & NA \\
\hline Receiver Tank Initial Height $(\mathrm{m})$ & 0.0 & NA & NA \\
\hline Receiver Tank Initial Density $\left(\mathrm{kg} / \mathrm{m}^{3}\right)$ & 0.0 & \pm 0.00254 & uniform \\
\hline Receiver Tank Final Height $(\mathrm{m})$ & 1.38 & \pm 47 & normal \\
\hline Receiver Tank Final Density $\left(\mathrm{kg} / \mathrm{m}^{3}\right)$ & 1147 & & \\
\hline $\begin{array}{l}\text { (a) NA denotes not applicable. } \\
\text { (b) Includes effect of mixer pump operation. }\end{array}$ & & \\
\hline
\end{tabular}

Herting (1997), Schreiber (1995), and Bingham et al. (1999). The uncertainties in the data, as well as their distributions, are also included. Depending on the instrumentation setup, different parameters will be measured. Where no data or uncertainties were available, the methods and effects discussed in Sections 4.2.2 and 4.2.5 were used. Instrumentation uncertainties for the different setups are included in the respective tables.

\subsubsection{Waste Material Balance Results}

The material balance errors due with variable waste properties and tank conditions were evaluated using Equations (2.22), (2.23), and (2.24). The Monte Carlo simulation approach was again used for error propagation. 
As discussed, three sets of measurements and three different transfer scenarios were considered. To facilitate the presentation and discussion of the results, these different cases are denoted in the manner outlined in Section 4.1.2, with an additional identifier of D to indicate a disturbed waste surface due to action of a mixer pump (applicable to transfer 3 ).

The variable waste properties and tank conditions error results for Transfers 1, 2, and 3 are presented in Table 4.9, 4.10, and 4.11, respectively. The 95\% confidence interval and 100\% interval (includes all outcomes given all input scenarios) for the largest material balance error are presented in the second and third columns. The percentage error of the $100 \%$ interval value compared to the total transfer is given in column 4. Columns 5 and 6 indicate the $95 \%$ confidence interval about the mean error.

Table 4.9. Material Balance Error for Transfer 1

\begin{tabular}{|l|l|l|c|c|c|}
\hline \multirow{2}{*}{\multicolumn{1}{c|}{ Case }} & \multicolumn{2}{c|}{ Error (gal) } & Error (\%) & \multicolumn{2}{c|}{ Error (gal) } \\
\cline { 2 - 6 } & $\mathbf{9 5 \%} \mathbf{C I}$ & $\mathbf{1 0 0 \%} \mathbf{I}$ & $\mathbf{1 0 0 \%} \mathbf{I}$ & U 95\% Mean CI & L 95\% Mean CI \\
\hline $\mathrm{A} / 1 / \mathrm{MM} 2$ & 44900 & 68400 & 7.2 & 5117 & 3932 \\
\hline $\mathrm{A} / 1 / \mathrm{Y} 2$ & 45000 & 68500 & 7.2 & 5137 & 3953 \\
\hline $\mathrm{B} / 1 / \mathrm{MM} 2$ & 9700 & 13600 & 1.5 & 5156 & 5029 \\
\hline $\mathrm{C} / 1$ & 44900 & 67200 & 7.0 & 5255 & 4074 \\
\hline
\end{tabular}

Table 4.10. Material Balance Error for Transfer 2

\begin{tabular}{||l|c|c|c|c|c||}
\hline \hline \multirow{2}{*}{ Case } & \multicolumn{2}{|c|}{ Error (gal) } & Error (\%) & \multicolumn{2}{c||}{ Error (gal) } \\
\cline { 2 - 6 } & $\mathbf{9 5 \%}$ CI & $\mathbf{1 0 0 \%}$ I & $\mathbf{1 0 0 \%}$ I & U 95\% Mean CI & L 95\% Mean CI \\
\hline $\mathrm{A} / 2$ & 22400 & 55100 & 8.2 & 405.7 & -213.3 \\
\hline $\mathrm{B} / 2 / \mathrm{MM} 2$ & 15000 & 30800 & 4.6 & 302.9 & -120.8 \\
\hline $\mathrm{C} / 2$ & 22200 & 55300 & 8.2 & 406.6 & -214.1 \\
\hline
\end{tabular}

Table 4.11. Material Balance Error for Transfer 3

\begin{tabular}{|c|c|c|c|c|c||}
\hline \multirow{2}{*}{ Case } & \multicolumn{2}{|c|}{ Error (gal) } & Error (\%) & \multicolumn{2}{c||}{ Error (gal) } \\
\cline { 2 - 6 } & $\mathbf{9 5 \%}$ CI & $\mathbf{1 0 0 \%}$ I & $\mathbf{1 0 0 \%}$ I & U 95\% Mean CI & L 95\% Mean CI \\
\hline $\mathrm{A} / 3$ & 6100 & 12500 & 8.3 & 108.9 & -59.6 \\
\hline $\mathrm{A} / 3 / \mathrm{D}$ & 7000 & 15600 & 10.2 & 127.6 & -66.9 \\
\hline $\mathrm{B} / 3 / \mathrm{MM} 2$ & 4300 & 7000 & 4.6 & 92.5 & -26 \\
\hline $\mathrm{C} / 3$ & 6100 & 12500 & 8.4 & 115.8 & -51.4 \\
\hline $\mathrm{C} / 3 / \mathrm{D}$ & 7200 & 15800 & 10.3 & 138.2 & -58.8 \\
\hline
\end{tabular}

\subsubsection{Evaluation}

Material balance errors on the order of $8 \%$ of the total transfer are expected with variable waste properties and tank conditions. The 100\% interval error for Transfer 1 ranged from 13,600 to 68,500 gallons, for Transfer 2 from 30,800 to 55,700 gallons, and for Transfer 3 from 7,000 to 15,800 gallons. Notice that, for each transfer, the upper and lower $95 \%$ confidence intervals about the mean show that the mean error is skewed to the positive side. For Transfer 1, the mean 
is always positive. A positive error indicates a gain in mass. The variable waste properties and tank conditions can therefore potentially mask a leak or misrouting. For each transfer, measurement set B, which had less reliance on height measurements, produced the lowest errors.

\subsection{Alternative Instrumentation Setup}

It was shown in the previous section that the introduction of additional uncertainty into the height measurements, be it from density changes, surface irregularities, disturbance of the waste surface, or retention of waste material on the tanks walls, significantly and adversely affects the material balance errors. For each transfer considered, decreased reliance on level measurements produced smaller errors. An alternative instrumentation setup with mass flow meters at the beginning and end of the transfer line is therefore considered. The best results will be achieved with either the Micro Motion Elite CMF 200 or T150.

Installation of a mass flow meter at the end of the transfer line will accomplish the following:

- Eliminate need for reliance on in-tank height measurements

- Allow waste density to be monitored

- Provide redundancy on the material balance

- Allow uncertainty to be determined more precisely.

\subsubsection{Material Balance Equations}

Mass flow meters at the beginning and end of the transfer line are expected to improve the material balance error. A schematic of the alternative instrumentation setup is shown in Figure 4.6.

In addition to significantly improving the material balance accuracy, including the mass flow meter at the end of the transfer line provides redundancy on the material balance. A material balance can be conducted between the two mass flow measurements in the pipe. Additionally, material flow measurements of the water and feed waste can be balanced against the flow meter at the beginning of the pipe, and material flow measurements from the receiver tank can be balanced against the flow meter at the end of the pipe.

The applicable material balance equations for the alternative instrumentation setup are presented below. All variables are as denoted in Section 2. The material balance error for the pipeline is

$$
\mathrm{E}=\int_{\mathrm{t}=\mathrm{t}_{0}}^{\mathrm{t}=\mathrm{t}_{1}} \rho_{\mathrm{FW}} \mathrm{Q}_{\mathrm{FW}} \mathrm{dt}-\int_{\mathrm{t}=\mathrm{t}_{0}}^{\mathrm{t}=\mathrm{t}_{1}} \rho_{\mathrm{PD}} \mathrm{Q}_{\mathrm{PD}} \mathrm{dt}
$$

which simplifies to

$$
\mathrm{E}=\rho_{\mathrm{FW}} \mathrm{Q}_{\mathrm{FW}} \mathrm{t}_{1}-\rho_{\mathrm{PD}} \mathrm{Q}_{\mathrm{PD}} \mathrm{t}_{1}
$$




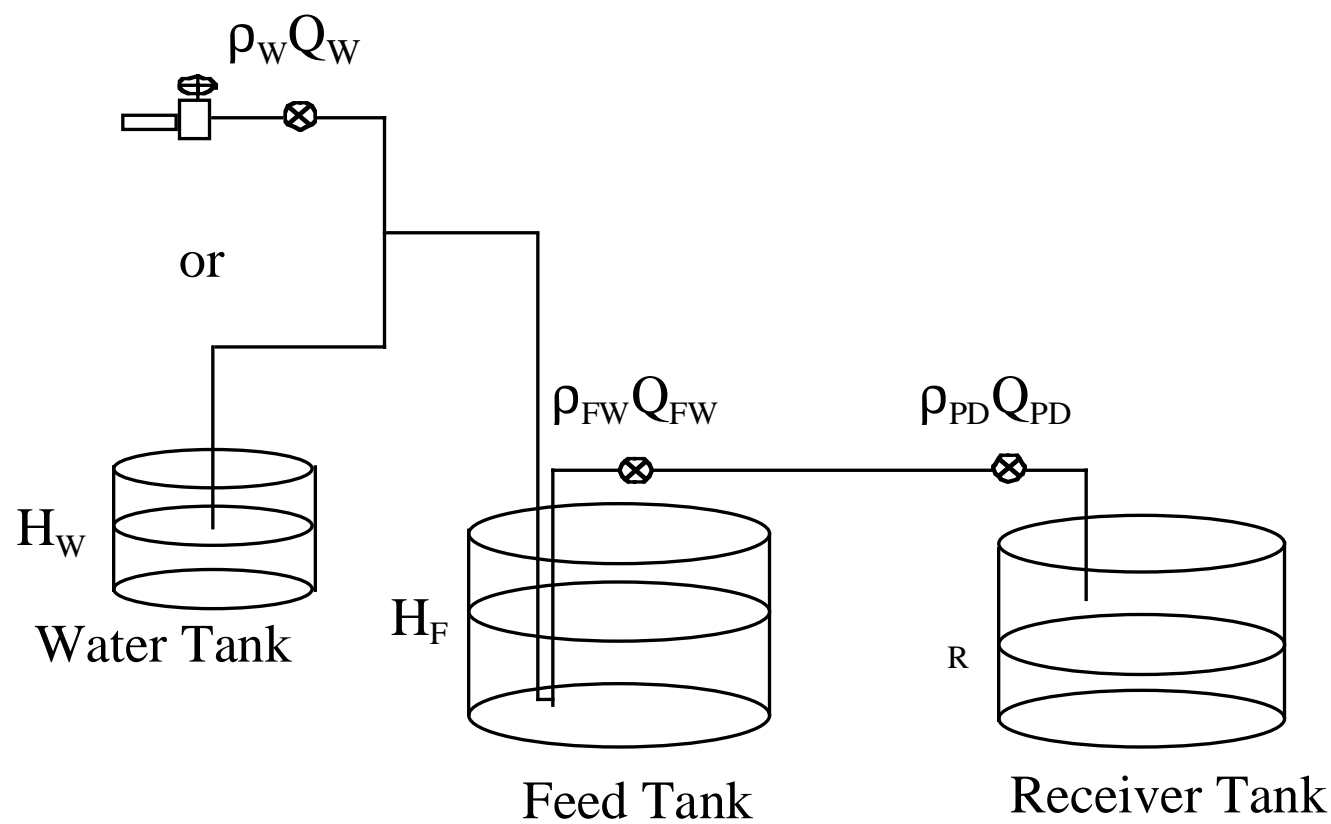

Figure 4.6. Alternative Instrumentation Setup

The material balance error for the material flow of the water and feed waste and the flow meter at the beginning of the pipe is

$$
\mathrm{E}=\int_{\mathrm{t}=\mathrm{t}_{0}}^{\mathrm{t}=\mathrm{t}_{1}} \rho_{\mathrm{w}} \mathrm{Q}_{\mathrm{w}} \mathrm{dt}-\int_{\mathrm{H}_{\mathrm{F}}=\mathrm{H}_{\mathrm{F} 0}}^{\mathrm{H}_{\mathrm{F}}=\mathrm{H}_{\mathrm{F} 1}} \mathrm{~A}_{\mathrm{F}} \rho_{\mathrm{F}} \mathrm{dH} \mathrm{H}_{\mathrm{F}}-\int_{\mathrm{t}=\mathrm{t}_{0}}^{\mathrm{t}=\mathrm{t}_{1}} \rho_{\mathrm{FW}} \mathrm{Q}_{\mathrm{FW}} \mathrm{dt}
$$

or

$$
\mathrm{E}=-\int_{\mathrm{H}_{\mathrm{W}}=\mathrm{H}_{\mathrm{W}_{0}}}^{\mathrm{H}_{\mathrm{W}}=\mathrm{H}_{\mathrm{W}_{1}}} \rho_{\mathrm{W}} \mathrm{dH} \mathrm{H}_{\mathrm{W}}-\int_{\mathrm{H}_{\mathrm{F}}=\mathrm{H}_{\mathrm{F}}}^{\mathrm{H}_{\mathrm{F}}=\mathrm{H}_{\mathrm{F}}} \mathrm{H}_{\mathrm{F}} \rho_{\mathrm{F}} \mathrm{dH} \mathrm{H}_{\mathrm{F}}-\int_{\mathrm{t}=\mathrm{t}_{0}}^{\mathrm{t}=\mathrm{t}_{1}} \rho_{\mathrm{FW}} \mathrm{Q}_{\mathrm{FW}} \mathrm{dt}
$$

Equations (4.3) and (4.4) simplify to

$$
\mathrm{E}=\rho_{\mathrm{W}} \mathrm{Q}_{\mathrm{W}} \mathrm{t}_{1}+\mathrm{A}_{\mathrm{F}} \rho_{\mathrm{F}}\left(\mathrm{H}_{\mathrm{F} 0}-\mathrm{H}_{\mathrm{F} 1}\right)-\rho_{\mathrm{FW}} \mathrm{Q}_{\mathrm{FW}} \mathrm{t}_{1}
$$

and

$$
\mathrm{E}=\mathrm{A}_{\mathrm{W}} \rho_{\mathrm{W}}\left(\mathrm{H}_{\mathrm{W} 0}-\mathrm{H}_{\mathrm{W} 1}\right)+\mathrm{A}_{\mathrm{F}} \rho_{\mathrm{F}}\left(\mathrm{H}_{\mathrm{F} 0}-\mathrm{H}_{\mathrm{F} 1}\right)-\rho_{\mathrm{FW}} \mathrm{Q}_{\mathrm{FW}} \mathrm{t}_{1}
$$

respectively. Likewise, the material balance error for the material flow of the receiver tank and the flow meter at the end of the pipe is

$$
E=\int_{H_{R}=H_{R} 0}^{H_{R}=H_{R} 1} A_{R} \rho_{R} d H_{R}-A_{R} \rho_{R 0} H_{R 0}-\int_{t=t_{0}}^{t=t_{1}} \rho_{P D} Q_{P D} d t
$$


which simplifies to

$$
\mathrm{E}=\mathrm{A}_{\mathrm{R}}\left(\rho_{\mathrm{R} 1} \mathrm{H}_{\mathrm{R} 1}-\rho_{\mathrm{R} 0} \mathrm{H}_{\mathrm{R} 0}\right)-\rho_{\mathrm{PD}} \mathrm{Q}_{\mathrm{PD}} \mathrm{t}_{1}
$$

\subsubsection{Results and Evaluation}

The material balance errors for the alternative instrumentation setup were determined under the variable waste properties and tank conditions presented in Section 4.2.6. Three error calculations for each transfer were conducted as follows:

P. Error between the two mass flow meters [Equation (4.2)]

F. Error between material flow measurements of the water and feed waste and the flow meter at the beginning of the pipe [Equation (4.5) or (4.6)]

R. Error between material flow measurements of the receiver tank and the flow meter at the end of the pipe [Equation (4.8)]

The different cases are again denoted in the manner outlined in Section 4.1.2. The results for the pipeline are presented in Table 4.12, and the results for all three error calculations are presented in Table 4.13.

Table 4.12. Pipeline Material Balance Error, All Transfers

\begin{tabular}{|l|c|c|c|c|c||}
\hline \multirow{2}{*}{ Case } & \multicolumn{2}{|c|}{ Error (gal) } & Error (\%) & \multicolumn{2}{c||}{ Error (gal) } \\
\cline { 2 - 6 } & $\mathbf{9 5 \%}$ CI & $\mathbf{1 0 0 \%}$ I & $\mathbf{1 0 0 \%}$ I & U 95\% Mean CI & L 95\% Mean CI \\
\hline P/1/MM2 & 2900 & 3900 & 0.4 & 42 & -41.9 \\
\hline P/2/MM2 & 2100 & 2900 & 0.4 & 30.9 & -30.7 \\
\hline P/3/MM2 & 500 & 900 & 0.6 & 7.6 & -7.6 \\
\hline
\end{tabular}

The error in the pipeline ranges from 3,900 gallons for Transfer 1 to 2,900 gallons for Transfer 2 and to 900 gallons for Transfer 3. These errors represent less than $1 \%$ of the total transfer volumes.

Table 4.13. Alternative Instrumentation Setup Material Balance Error, All Transfers

\begin{tabular}{|l|c|c|c|c|c||}
\hline \multirow{2}{*}{\multicolumn{1}{|c|}{ Case }} & \multicolumn{2}{|c|}{ Error (gal) } & Error (\%) & \multicolumn{2}{c||}{ Error (gal) } \\
\cline { 2 - 6 } & $\mathbf{9 5 \%}$ CI & $\mathbf{1 0 0 \%} \mathbf{I}$ & $\mathbf{1 0 0 \%} \mathbf{I}$ & U 95\% Mean CI & L 95\% Mean CI \\
\hline P/1/MM2 & 2900 & 3900 & 0.4 & 42 & -41.9 \\
\hline F/1/MM2 & 38300 & 61800 & 6.5 & 118.3 & -1021.2 \\
\hline R/1/MM2 & 9900 & 13500 & 1.5 & 5216.9 & 5089.3 \\
\hline P/2/MM2 & 2100 & 2900 & 0.4 & 30.9 & -30.7 \\
\hline F/2/MM2 & 15600 & 29700 & 4.4 & 205.9 & -225.6 \\
\hline R/2/MM2 & 15900 & 30800 & 4.6 & 307.6 & -124.4 \\
\hline P/3/MM2 & 500 & 900 & 0.6 & 7.6 & -7.6 \\
\hline F/3/MM2 & 4000 & 6900 & 4.6 & 57.3 & -57.2 \\
\hline F/3/MM2/D & 5600 & 10600 & 6.9 & 73.6 & -79 \\
\hline R/3/MM2 & 4300 & 7000 & 4.7 & 85 & -31.9 \\
\hline
\end{tabular}


The material balance errors in the pipe are much less than the feed and receiver tank errors for each of the transfer scenarios. Comparison of the material balance errors in Table 4.13 to those in Tables 4.9, 4.10, and 4.11 clearly demonstrates the significant improvement in the achievable material balance accuracy for the alternative instrumentation setup. The apparent material balance error in the feed and receiver tanks is also reduced by the direct comparison of the tank material to a flow meter. Again, the positive mean errors indicated by the $95 \%$ confidence interval about the mean can potentially mask leaks or mis-routings.

The redundancy in the material balance calculations for the alternative instrumentation setup also provides the ability to check and maintain instrumentation accuracy. For example, if the material balances for the feed and receiver tanks and the flow meter at the beginning of the pipe indicate no error, but the flow meter at the end of the pipe indicates error, that is a strong indication that the flow meter at the end of the pipe is not operating correctly. Likewise, if a tank measurement is not in agreement with combinations of the other measurements, instrumentation inaccuracy or in-tank effects are indicated.

\subsubsection{Transfer System Implications of Alternative Instrumentation Setup}

Implementation of the alternative instrumentation setup requires additional mass flow meters to be installed at the receiver tank end of the transfer line. It is planned to have flow meters for the outflow of each tank; therefore, modification of the waste flow path would be required. The Micro Motion meters allow the flow rate to be measured for flow in either direction through the meter with no loss in accuracy.

Some engineering aspects of implementing an additional mass flow meter are discussed below. The process flow specifications are 100 to $164 \mathrm{gpm}$ with a specific gravity of 1.1 to 1.4 , which yields a flow rate range of approximately 1000 to $2000 \mathrm{lb} / \mathrm{min}$. The head loss through the flow meters has been estimated for the higher flow rate of $164 \mathrm{gpm}$, which equates to $7.2 \mathrm{ft} / \mathrm{sec}$ in a Sch-40 3-in. pipe. The head losses are listed in Table 4.14. The space requirement for the Micro Motion CMF200 and CMF300 are approximately 7 in. x 29 in x 23 in. and 10 in. x 39 in. x 36 in., respectively. The Micro Motion T150 housing is approximately 32 in. long and $7.2 \mathrm{in.}$ in diameter.

Table 4.14. Mass Flow Meter Head Loss

\begin{tabular}{|l|c|c|}
\hline \multicolumn{1}{|c|}{ Meter } & $\begin{array}{c}\text { Mass Flow Rate Range } \\
\text { (lb/min) }\end{array}$ & $\begin{array}{c}\text { Head Loss Across Unit at 164 gpm } \\
\text { (ft) }\end{array}$ \\
\hline CMF200 U-tube & 0 to 3,200 & $24^{\text {(a) }}$ \\
\hline CMF300 U-tube & 0 to 10,000 & 3 \\
\hline T150 Straight tube & 0 to 3,200 & $10^{(\text {a) }}$ \\
\hline (a) Does not account for contraction and expansion from 3-in. pipe. \\
\hline
\end{tabular}




\subsection{Summary and Conclusions}

We developed a material balance assessment methodology based on conservation of mass to address the pipeline transfer of DST tank waste with variable waste properties and tank conditions. The methodology consists of a set of material balance equations and general approaches to solve them, including how to determine waste density changes due to chemical reactions and gas generation/retention/release. It is intended to be a backup to pit leak detectors to detect waste leaking and mis-routings during waste transfer between and within the 200 East and 200 West Areas, including the planned vitrification plant.

The main factors causing variable waste properties and tank conditions are waste density changes caused by chemical reactions and gas generation/retention/release, the existence of a crust layer and its potential effects on density, waste height and tank cross-sectional area, and waste surface disturbance due to mixer pump operation during the waste transfer. Without these effects, this mass-based material balance methodology is simplified to the traditional volumebased material balance approach for constant waste properties and tank conditions. Thus, the latter is applicable to tanks without these complex factors, such as AP Tank Farm waste transfers.

The material balance assessment methodology was applied to three waste transfers: AN-105 first transfer of 911,400 gallons of in-line diluted supernatant liquid, AN-105 second transfer with 673,000 gallons of liquid waste, and AZ-102 slurry transfer with 150,000 gallons. Three instrumentation setups were considered: A) feed and receiver tank levels and diluent flow meter, B) flow meter at the beginning of the transfer pipeline and receiver tank level, and C) diluent, feed, and receiver tank levels.

Monte Carlo simulations to determine the potential waste transfer material balance errors due to the instrumentation indicate that the errors are relatively small (up to 2,200 gallons for AN-105 first transfer with instrumentation setup B, having a Micro Motion Elite CMF 200 or T150 mass flow meter), typically accounting for less than $1 \%$ of the total transfer. This error is small enough to be able to identify the error due to effects other than actual leakage or misrouting. Specific amounts of material balance error for the instrumentation in each waste transfer case are summarized below:

- For the AN-105 first liquid waste transfer, errors range from 1,000 gal ( $0.11 \%$ error) with Enraf surface measurements in feed, water and receiver tanks (instrumentation setup C) to 2,200 gal with the Micro Motion mass flow meter (Elite CMF 200 or T150) and Enraf surface measurement in the receiver tank (instrumentation setup B).

- For the AN-105 second liquid transfer to DSTs, errors range from to 800 gal $(0.12 \%)$ with all Enraf measurements (instrumentation setup C) to 1,600 gal $(0.11 \%)$ with the Micro Motion mass flow meter (Instrumentation Setup B). For transfer to the vitrification plant storage tanks, these errors ranges from 1,600 gal (0.24\%) with instrumentation setup B to 1,900 gal $(0.28 \%)$ with instrumentation setups $\mathrm{A}$ and $\mathrm{C}$.

- For the AZ-102 slurry transfer, errors vary from 600 gal $(0.38 \%)$ with the Micro Motion mass flow meter (instrumentation setup B) to 800 gal $(0.52 \%)$ with all Enraf measurements (instrumentation setups $\mathrm{A}$ and $\mathrm{C}$ ). For the vitrification plant storage tanks, these errors 
ranges from 400 gal $(0.24 \%)$ with instrumentation setup B to 1,100 gal $(0.75 \%)$ with instrumentation setups A and C.

Height measurements alone generally produced lower material balance errors. However, the variable waste properties and tank conditions that are to be expected during the transfer of saturated wastes make the height measurements subject to a significant error. When uncertainties due to variable waste properties and tank conditions were included in the analysis, the material balance errors became much larger, as summarized below.

- For the AN-105 first liquid waste transfer, errors range from 13,600 gal (1.5\%) with instrumentation setup B to 68,400 gal (7.2\%) with instrumentation setup C.

- For the AN-105 second liquid transfer, errors range from 30,800 gal (4.6\%) with instrumentation setup B to 55,300 gal (8.2\%) with instrumentation setup C.

- For the AZ-102 slurry transfer, errors vary from 7,000 gal (4.6\%) with instrumentation setup B to $15,800 \mathrm{gal}(10.3 \%)$ with instrumentation setup C.

An alternative instrumentation setup to reduce these errors is to have a Micro Motion Elite CMF 200 or T150 mass flow meter at the both ends of the transfer pipeline. Installation of these mass flow meters at the both end of the transfer line will serve to

- eliminate reliance on in-tank height measurements

- allow waste density to be monitored

- provide redundancy on the material balance

- allow uncertainty to be determined more precisely.

Under this alternative instrumentation setup, the material balance error in the transfer pipeline is

- for the AN-105 first liquid transfer, 3,900 gallons (0.4\%).

- for the AN-105 second liquid transfer, 2900 gallons (0.4\%).

- for the AZ-102 slurry transfer, 900 gallons $(0.6 \%)$.

Thus, depending on the operational needs, one can select

- volume-based material balance equations for constant waste properties and tank conditions

- mass-based material balance equations for variable waste properties and tank conditions with one or more combinations of the three instrumentation setups (A, B, and $\mathrm{C}$ )

- mass-based material balance equations for variable waste properties and tank conditions with the alternative instrumentation setup having Micro Motion mass flow meters at both ends of the transfer pipeline. 


\subsection{References}

Bingham JD, SA Barker, WB Barton, DC Hedengren, and TA Hu. 1999. Gas Release Event Safety Analysis Tool Pedigree Database for Hanford Tanks. HNF-SD-WM-TI-806, Rev 2B, Mactec-Meier Associates LLC, Richland, Washington.

Enderlin CW, G Terrones, and OD Mullen. 1997. Performance Evaluation of the Quarter-Scale Russian Retrieval Equipment for the Removal of Hazardous Waste. PNNL-11740, Pacific Northwest National Laboratory, Richland, Washington.

Enderlin CW, G Terrones, and B Hatchell. June 2002. "Scaled Testing to Determine Operational Strategies for Removal of Zeolite in Savannah River Site Tank 19 Using Shrouded Axial Impeller Mixers." Proceedings of 15th International Conference on HydroTransport, Banff, Canada. BHR Group Limited, Bedfordshire, U.K., Vol. I, pp. 187-201.

Estey SD. 2000. Process Control Plan for Tank 241-SY-101 Surface Level Rise Remediation. HNF-4264, Rev. 3B. CH2M Hill Hanford Group, Inc. Richland, Washington.

Felmy AR. 1995. “GMIN, A Computerized Chemical Equilibrium Program Using a Constrained Minimization of the Gibbs Energy: Summary Report." Chemical Equilibrium and Reaction Models, Soil Science Society of America, Special Publication 42.

Greenwood MS and JA Bamberger. 2000. Measuring Slurry Density and Viscosity In Situ in Real time During Transport Using an Ultrasonic Sensor. FEDSM00-11121, ASME 2000 Fluids Engineering Division Summer Meeting, Boston. American Society of Mechanical Engineers, New York.

Hedengren DC, KM Hodgson, WB Barton, CW Stewart, JM Cuta, and BE Wells. 2000. Data Observations on Double-Shell Flammable Gas Watch List Tank Behavior. RPP-6655 Rev. 0, CH2M HILL Hanford Group, Inc., Richland, Washington.

Herting DL. 1997. Results of Dilution Studies with Waste from Tank 241-AN-105. HNF-SDWM-DTR-046, Rev. 0, Numatec Hanford Corporation, Richland, Washington.

Herting DL. 1998. Results of Dilution Studies with Waste from Tank 241-AN-104. HNF-3352, Rev. 0, Numatec Hanford Corporation, Richland, Washington.

Hylton TD and CK Bayne. 1999. Testing of In-Line Slurry Monitors and Pulsair Mixers with Radioactive Slurries. ORNL/TM-1999/111, Oak Ridge National Laboratory, Oak Ridge, Tennessee.

Kirch NW, JM Conner, WB Barton, CW Stewart, BE Wells, and JM Grigsby. 2000. Remediation of Crust Growth and Buoyant Displacement Gas Release Event Behavior in Tank 241-SY-101. RPP-6754, CH2M HILL Hanford Group, Inc., Richland, Washington.

Mahoney LA, ZI Antoniak, JM Bates, and ME Dahl. 1999. Retained Gas Sampling Results for the Flammable Gas Program. PNNL-13000, Pacific Northwest National Laboratory, Richland, Washington.

Mahoney LA, ZI Antoniak, WB Barton, JM Conner, NW Kirch, CW Stewart, and BE Wells. 2000. Results of Waste Transfer and Back-Dilution in Tanks 241-SY-101 and 241-SY-102. PNNL-13267, Pacific Northwest National Laboratory, Richland, Washington. 
Onishi Y, KP Recknagle, and BE Wells. 2000. "Pump Jet Mixing and Pipeline Transfer Assessment for High-Activity Radioactive Wastes in Hanford Tank 2451-AZ-102." PNNL13275, Pacific Northwest National Laboratory, Richland, Washington.

Powell, MR, BK Hatchell, WH Combs, MA Johnson, JR Farmer, MR Poirier, H Gladki, PO Rodwell. 1999. Evaluation of Flygt Mixers for Application in Savannah River Site Tank 19, Test Results from Phase B: Mid-Scale Testing at PNNL. PNNL-12093, Pacific Northwest National Laboratory, Richland, Washington.

Rassat SD, CW Stewart, BE Wells, WL Kuhn, ZI Antoniak, JM Cuta, KP Recknagle, G Terrones, VV Viswanathan, JH Sukamto, and DP Mendoza. 2000. Dynamics of Crust Dissolution and Gas Release in Tank 241-SY-101. PNNL-13112, Pacific Northwest National Laboratory, Richland, Washington.

Reynolds BA, EA Daymo, JGH Geeting, and J Zhang. 1996. Instrumentation Validation Project. PNNL-11221, Pacific Northwest National Laboratory, Richland, Washington.

Schreiber RD. 1995. Tank Characterization Report for Double-Shell Tank 241-AZ-102. WHC-SD-WM-ER-411 Rev. 0-A, Westinghouse Hanford Company, Richland, Washington. 
Appendix A

The Micro Motion TSM Calibration System 


\section{The Micro Motion TSM Calibration System}

\section{Introduction}

The Micro Motion TSM calibration system is the latest advance in Micro Motion's calibration capabilities. This system provides a new level of automation and measurement assurance to the calibration of Micro Motion and Fisher-Rosemount flow products that gives customers even greater consistency and quality in the measurements they receive when they choose Fisher-Rosemount products.

This description of the TSM calibration system is intended to answer some of the questions people ask about how it works and why it is known to be accurate.

\section{System Layout}

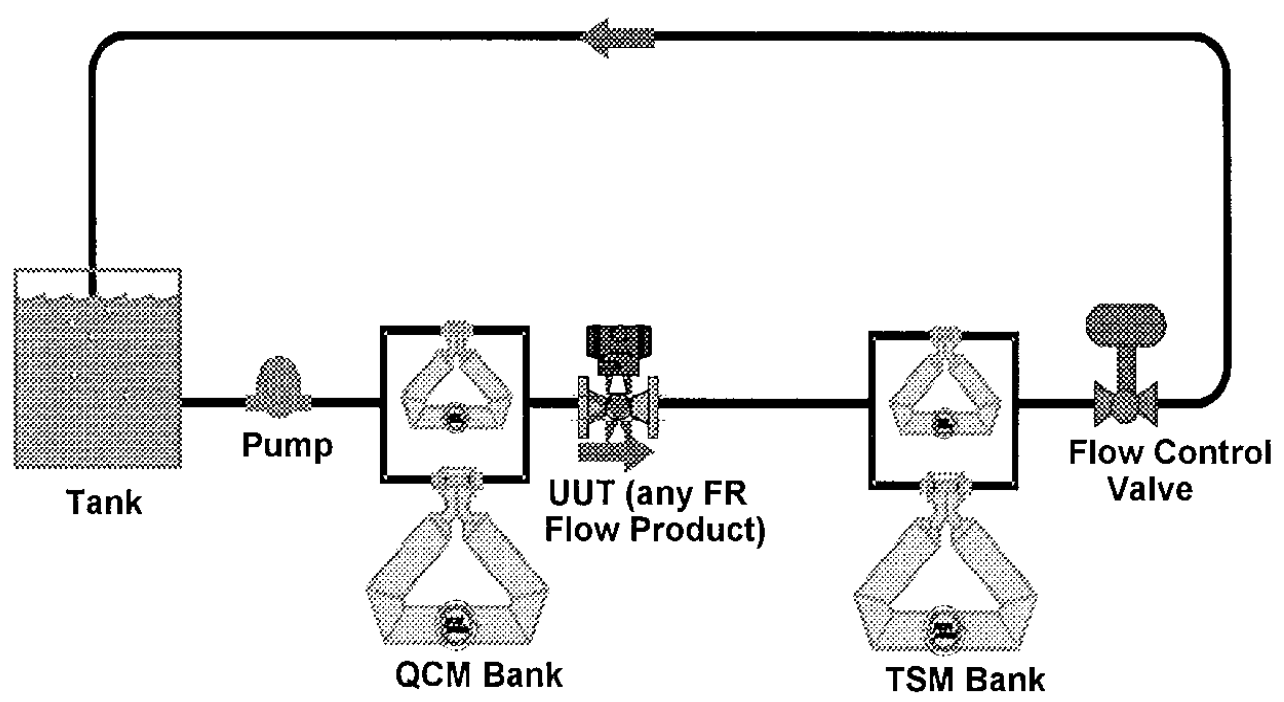

Figure 1 - TSM system layout

Figure 1 is a diagram of the TSM system layout. The flow is pumped from the tank into the QCM bank of meters. QCM stands for Quality Check Meter, and these meters serve as check standards to verify the TSM meters and the system accuracy during every point that is taken. Several parallcl paths exist in the QCM bank each with a different size meter. All these paths, except the one that has the most accurate size flow meter for the chosen flow rate, are automatically blocked.

After passing through the QCM bank, the flow is directed through the UUT (Unit Under Test). The test unit section of the TSM system is designed to accommodate two UUT flow meters in scries. System pressure and temperature are measured in the test section, as well as at other points throughout the system not shown in Figure 1. The location of the test section between the TSM and QCM banks allows the system to detect any leakage that would effect the accuracy of the UUT calibration by comparing the TSM meter results against the QCM meter results for each point measured. 
After passing through the test section, the flow continues through the TSM bank. TSM stands for Transfer Standard Meter, and these meters serve as the flow reference to which the UUT is compared. The TSM bank consists of a set of parallel paths identical to those in the QCM bank. Flow is directed through the same path as in the QCM bank, with all others automatically blocked. The UUT is compared only to the TSM meter, and that comparison is allowed only if the check of the TSM meter against the QCM meter is first verified for that same point.

After the TSM bank, flow continues through a control valve. The control valve and the variable speed pump control the system flow rate and pressure. Flow then returns directly to the system tank.

\section{Calibration of Coriolis Meters}

\section{Transmitter setup}

Before a Coriolis meter is calibrated on the TSM system, the meter transmitter is automatically set up for calibration by configuring the following parameters:

- Frequency output scaling

- Approximate flow calibration factor

- Flow temperature compensation coefficient

- Flow cutoff

- Flow damping

- Approximate Density calibration factors

- Density temperature compensation coefficient

- Approximate FD factor

- All variable units of measure

\section{Density calibration}

\section{Air point calibration}

Before the Coriolis meter is filled with water, the air point density calibration is performed. The reference air density is calculated with equation E1 using measurements of the laboratory temperature, relative humidity, and barometric pressure.

Equation E1

$$
\rho_{\text {air }}=\left[0.0012929 \times\left(\frac{273.13}{T+273.14}\right)\right] \times\left[\frac{\left(51.715 \times B P-(0.473 \times T+2.856) X \frac{\% R H}{100}\right)}{760}\right]
$$

Where: $\mathrm{T}=$ tempcrature in ${ }^{\circ} \mathrm{C}$.

$\mathrm{BP}=$ barometric pressure in psi.

$\% \mathrm{RH}=$ Relative humidity in \% $(0-100)$

$\rho_{\text {air }}=$ reference density of air in $\mathrm{g} / \mathrm{cc}$.

The TSM system automatically assures temperature stability for the air point calibration. The calibration is not allowed to proceed until the test meter temperature and the laboratory temperature agree. 
After the air point calibration, the accuracy of the calibration is checked by comparing the test meter air density measurement to the reference air density calculated by Equation E1. The air point calibration is further checked by automatically comparing the $\mathrm{K} 1$ factor determined to the known range of $\mathrm{K} 1$ factors for the test meter model.

\section{Water point calibration}

After the air point density calibration, the meter is filled with water. The system is flushed automatically to remove all air. The automatic filling process does not stop until all the TSM and QCM meters in the system indicate the accurate density of water.

Before the water point density calibration is performed, the TSM system automatically assures temperature stability for the water point calibration. Temperature stability for the water point density calibration is confirmed by three separate checks. The test meter and two system temperatures may not vary outside of set control limits for one complete minute. The system temperature measurements up and down stream of the test meter must agree. The system temperature must be within the $6{ }^{\circ} \mathrm{C}$ allowable operating range. At this time, the accuracy of the test meter temperature is also verified.

The reference water density is calculated with equations E2, E3, E4, and E5 using the average temperature measured by the up and downstream temperature transmitters, and the system pressure measurements. The system temperature and pressure measurements are periodically checked against standards traccable to local standards bodies.

Equation E2

$$
\rho_{W t p}=\rho_{W t} /\left[1-\bar{K}_{t p}\left(P_{f}-P_{d}\right)\right]
$$

Where: $\mathrm{P}_{\mathrm{f}}=$ pressure in psia.

$$
P_{d}=14.696 \text { psia }(1 \mathrm{~atm} .)
$$

Equation E3

$$
\begin{aligned}
& \rho_{W t}=\left[\left(9.998395639 \times 10^{2}\right)+\left(6.798299989 \times 10^{-2}\right)\left(T_{f}\right)-\left(9.106025564 \times 10^{-3}\right)\left(T_{f}^{2}\right)\right. \\
& \left.+\left(1.005272999 \times 10^{-4}\right)\left(T_{f}^{3}\right)-\left(1.126713526 \times 10^{-6}\right)\left(T_{f}^{4}\right)+\left(6.591795606 \times 10^{-9}\right)\left(T_{f}{ }^{5}\right)\right] / 1000
\end{aligned}
$$

Where: $T_{f}=$ average between up and down stream temperatures in ${ }^{\circ} \mathrm{C}$

Equation E4

$$
\bar{K}_{t p}=K_{t}\left\langle 1.00033-\left[\left(2.17656 \times 10^{-5}\right)\left(P_{f}-P_{d}\right) / 2\right]+\left\{\left(8.546265 \times 10^{-10}\right)\left[\left(P_{f}+P_{d}\right) / 2\right]^{2}\right\}\right\rangle
$$

Where: $P_{\mathrm{f}}=$ pressure in psia

$$
P_{d}=14.696 \text { psia }(1 \mathrm{~atm} .)
$$

Equation E5

$$
\begin{aligned}
& K_{t}=\left[50.88496+\left(6.163813 \times 10^{-1}\right)\left(T_{f}\right)+\left(1.459187 \times 10^{-3}\right)\left(T_{f}^{2}\right)+\left(20.08438 \times 10^{-6}\right)\left(T_{f}^{3}\right)\right. \\
& \left.-\left(58.4772 \times 10^{-9}\right)\left(T_{f}^{4}\right)+\left(410.411 \times 10^{-12}\right)\left(T_{f}^{5}\right)\right] \\
& \div\left\{\left[1+\left(19.67348 \times 10^{-3}\right)\left(T_{f}\right)\right]\left(14.50377 \times 10^{6}\right)\right\}
\end{aligned}
$$

Where: $T_{f}=$ average betwecn up and down stream temperatures in ${ }^{\circ} \mathrm{C}$ 
The reference water density is corrected for the small effect due to impurities in the water using equation E6. The correction factor, APF, is determined by periodic sampling of the laboratory water. The water sample density is measured by a laboratory grade densimeter calibrated using a traceable density standard.

Equation E6

$$
\rho_{\text {ref }}=\rho_{W t p}+I A F
$$

Where: $\mathrm{IAF}=$ measured difference from distilled water due to impurities.

$\rho_{\mathrm{ref}}=$ reference density corrected for impurities.

After the water point calibration, the accuracy of the calibration is checked by comparing the test meter water density measurement to the reference water density calculated by Equations E2-E6. The water point calibration is further checked by automatically comparing the $\mathrm{K} 2$ factor determined to the known range of $\mathrm{K} 2$ factors for the test meter model and the difference $\mathrm{K} 2-\mathrm{K} 1$ to the known range for the test meter model.

\section{FD calibration}

The FD factor for correcting the effect of high flow rates on density measurement is calibrated after the air point and water point density calibrations are complete. The flow rate is set at $100 \%$ of the nominal flow rate for the test meter. The temperature stability is verified by the same three checks used prior to the water point density calibration. The water reference density is determined in the same manner as for the water point density calibration.

After the FD factor calibration, the accuracy of the calibration is checked by comparing the test meter water density measurement to the reference water density calculated by Equations E2-E6. The FD calibration is further checked by automatically comparing the FD factor determined to the known range of FD factors for the test meter model.

\section{Test Meter Zero Set}

Before any flow calibration is done, the flow is completely stopped and the test meter is zeroed.

\section{Flow Calibration}

\section{Flow references}

A primary calibration of each TSM and QCM meter is performed before they are installed in a TSM system. These calibrations are repeated cvery year. Primary calibrations are performed on a weigh scale system. The weigh scales are calibrated using mass standards traceable to both NIST and JCSS. Each primary calibration consists of 275 to 325 points in sets of 25 points at each flow rate. The total number of points collected depends on the size of the meter and the range of flow rates it covers.

The points from the primary calibrations are used to verify the repeatability and accuracy of each TSM and QCM meter, and to linearize the output for the greatest possible accuracy. A least squares polynomial regression analysis is done to determine the linearization coefficients for cach meter. TSM and QCM meter outputs are also corrected for known pressure effects using TSM system pressure measurements to eliminate every known source of type B error. The reference totals from TSM and QCM meters are in units of $\mathrm{kg}$. 


\section{Calibration points}

Each calibration point is measured by following this procedure:

1. Set the flow rate - The TSM system uses PID control loops within the DeltaV system control software to adjust the control valve positions and pump speed until the set point flow rate and pressure for the calibration point are reached. System temperature is constantly controlled within a $6{ }^{\circ} \mathrm{C}$ range by a Cromolox controller.

2. Freeze the flow rate - Once the system set points are reached, the system control is locked. The control valve positions and pump speed do not change again until the measurement is complete.

3. Start the pulse counters - Individual pulse counters measuring the outputs from the test meter and the active TSM and QCM meters are simultaneously triggered on. The triggering device has been designed and tested to confirm a maximum of one pulse difference between the individual counter totals at all frequencies and calibration point collection times used by the TSM system.

4. Monitor the process conditions - While pulses are accumulating in the counters, the TSM system constantly monitors the flow rate, pressure, and temperature. If any of these parameters drift outside of allowable limits, the counters are stopped and the calibration point is aborted.

5. Stop the pulse counters - The counters continue to accumulate pulses until the minimum calibration point collection time has elapsed, the minimum number of pulses needed from the test meter have been collected, and at least 50,000 pulses have been collected from the TSM meter. Then, the individual pulse counters are simultaneously triggered off. The accumulated pulse totals are converted into $\mathrm{kg}$ for comparison by using the frequency output scaling from the test meter and the active TSM and QCM meters.

6. Perform the QCM check of the TSM measurement - Before it is used to compare to the test meter total, the TSM reference total is compared to the QCM total. The comparison is made as the percent difference between the active TSM total and the active QCM total according to equation E7. This comparison is referred to as the "QCM check". If the QCM check for TSM meters one inch in size and smaller is greater than $\pm 0.03 \%$, then the calibration point is aborted. Two and three inch TSM meters allow a QCM check of $\pm 0.04 \%$ before the calibration point is aborted.

Equation E7

$$
\mathrm{QCM} \text { check point }=\frac{(T S M-Q C M)}{Q C M} \times 100 \%
$$

7. Calculate the difference between the test meter and the reference meter - If the QCM check is passed, the value of the calibration point is determined as the percent difference between the totals from the test meter and the active TSM meter according to equation E8.

Equation E8

$$
\text { Test meter calibration point }=\frac{(\text { Test }- \text { TSM })}{\text { TSM }} \times 100 \%
$$

\section{Test Meter Calibration and Adjustment}

Three calibration points are taken for each test meter at the $100 \%$ nominal flow rate for that meter. The average of those three points is used to calculate a new flow calibration factor for the test meter to replace the approximate factor entered during the transmitter setup. The flow calibration factor in the transmitter configuration is adjusted to the new value before the test meter is verified.

The standard deviation of the three points is automatically compared to the repeatability specification of the test meter. If the check is unsuccessful, the test meter calibration is stopped. 


\section{Test Meter Verification}

After the density and flow calibration factors have been adjusted as described above, the test meter is verified by a standard pattern of calibration points. The standard pattern contains points at $100 \%, 10 \%$, $50 \%$, and a second at $100 \%$ of the nominal flow rate for that meter. The flow accuracy, density accuracy, and temperature accuracy of the test meter are all checked during each of these calibration points.

The TSM system can accommodate patterns other than the standard calibration pattern so that custom calibration requests can be met.

In order for the calibration verification points to pass, the resulting value must be within the allowance defined by the test meter accuracy specification minus the TSM system uncertainty at the test flow ratc. If passing points for each of the calibration points called for by the verification pattern do not result, the test meter calibration has failed.

\section{The Measurement Assurance Process}

\section{QCM check data review}

All the QCM check data that is collected is reviewed by the laboratory support engineers each month. The engineers analyze the data to ensure the calibration process variation is within expected limits.

\section{Gold meter testing}

A gold meter is a normal flow meter that has been set aside for a weckly check of the TSM system. The gold meter is used each week to confirm the stability of the TSM system. There is nothing special about the gold meter other than that it is the exact same metcr cach week. The gold meter is calibrated by the TSM system, and the results are statistically analyzed to verify that it does not change over time.

Each gold meter is used to cover multiple flow rates and multiple systems within the same laboratory. This provides overlap between the existing systems within each laboratory. Each laboratory will have gold meters of different sizes, however, to provide the most repeatable flow measurcment at cach gold meter test flow rate.

\section{Measurement comparison program}

Each TSM stand is included in a measurement comparison program every year. The measurement comparison program is a laboratory inter-comparison that involves testing a test meter in multiple laboratories and systems in order to compare the results for agreement

\section{Calibration of Volumetric Meters}

Volumetric meters, like magnetic and vortex, are calibrated in the same way as Coriolis meters except that the reference totals are converted from $\mathrm{kg}$ into volumetric units of liters using the reference water density calculated by equations E2 - E6. The TSM system follows the calibration point flow rate patterns and adjustment procedures unique to each technology. 


\section{Appendix B}

Traceability and Uncertainty Analysis for a Calibration Process for Flowmeters, Using Coriolis Flowmeters as Reference 


\title{
Appendix B: \\ Traceability and Uncertainty Analysis for a Calibration \\ Process for Flowmeters, Using Coriolis Flowmeters as Reference
}

\author{
Robert DeBoom, Marc Buttler, Andrew Kolbeck \\ Micro Motion, Inc. \\ Boulder, Colorado, USA \\ Aart Pruysen \\ Fisher-Rosemount \\ Veenendaal, NL
}

\begin{abstract}
A flow meter manufacturer has recently developed a new system for calibrating its flow instruments. This system is based on the use of Coriolis flow meters as intermediate transfer standards. This new system is referred to as the Transfer Standard Method or TSM.
\end{abstract}

This paper and presentation will review the test data used to establish the validity of the Coriolis-based TSM, and will describe the process layout and design of the new TSM flow calibration stands. The focus of the paper, however, will be on traceability of the calibrations to national standards and on the uncertainty analysis for the TSM flow stands.

The working mass standards in the MMI (Micro Motion, Inc.) Primary Stand laboratory are traceable to Japanese and United States of America national standards. These working mass standards are used to calibrate and verify the scales of the MMI Primary Stand. The MMI Primary Stand is used to calibrate the reference meters. The reference meters are placed into service in TSM stands.

The uncertainty analysis can be split into four main parts:

A. Uncertainty of the scales in the MMI Primary Stand

B. Uncertainty of the MMI Primary Stand (Best Measurement Capability of the MMI Primary Stand)

C. Uncertainty of the reference meters, calibrated on the primary stand

D. Uncertainty of the TSM stand (Best Measurement Capability of TSM stand).

This paper and presentation explain, step by step, the uncertainty calculations from scale to flowmeter under test. The end result of this analysis, for worst case conditions, is a Best Measurement Capability of the TSM system of $0.013 \%$ on mass and $0.020 \%$ on volume.

Keywords: Coriolis, traceability, uncertainty, transfer standard, flow meter, best measurement capability 


\section{INTRODUCTION}

The three purposes of this paper are:

- To discuss flowmeter calibration stand requirements and data used by the flowmeter manufacturer to support the development of the TSM (transfer standard method) calibration stand and to describe how the new TSM calibration stands operate.

- To review traceability for the new TSM calibration stands.

- To describe the TSM stand uncertainty analysis.

\section{FLOWMETER CALIBRATION STAND REQUIREMENTS}

The calibration requirements for the manufacturer's flowmeter calibration stands include:

- Uncertainty of $<0.033 \%$ mass. (This value of stand uncertainty is required in order to achieve a 3 times capability when calibrating flowmeters that have specified reference accuracy of $0.10 \%$.)

- Mass and volumetric calibration

- Straight run for velocity type meters

- Readily automated

- Minimum calibration time

- Low maintenance

- Worldwide consistency

\section{FEASIBILITY STUDY}

The calibration technologies considered included gravimetric SSF (standing start-finish), gravimetric diverter and small volume prover. The TSM design was developed after collecting and reviewing data from the manufacturer's CMF series Coriolis sensors. The data was collected by using a special calibration on the manufacturer's standard CMF series flowmeters. The data for figure 1 were obtained using a gravimetric SSF calibration stand and the manufacturer's CMF

The history of the stability of the CMF series sensors "flow calibration factor" is excellent. The flow calibration factors for a number of sensors have been recorded for 4 or more years and there is no change in the flow calibration factors within the uncertainty of the flowmeter calibration process. Stability of the flow calibration factor for CMF series sensors is an active area of continuing study.

Note that the data shown in figure 1 (as well as all further references to performance of Coriolis meters throughout this paper) are from standard production MMI (Micro Motion, Inc.) Elite meters. The only thing special about these meters is their calibration; their design and fabrication are, in all other respects, identical to all other MMI Elite meters.

The data shown in figure 1 support that, over a range of flow rates, standard CMF sensors can be calibrated, with linearization, to a very low type A uncertainty (repeatability), much better than the meter's standard reference specification for flow, $0.10 \%$. These specially calibrated sensors are then used as the RM (reference meter(s)) in the TSM flowmeter calibration stands. 


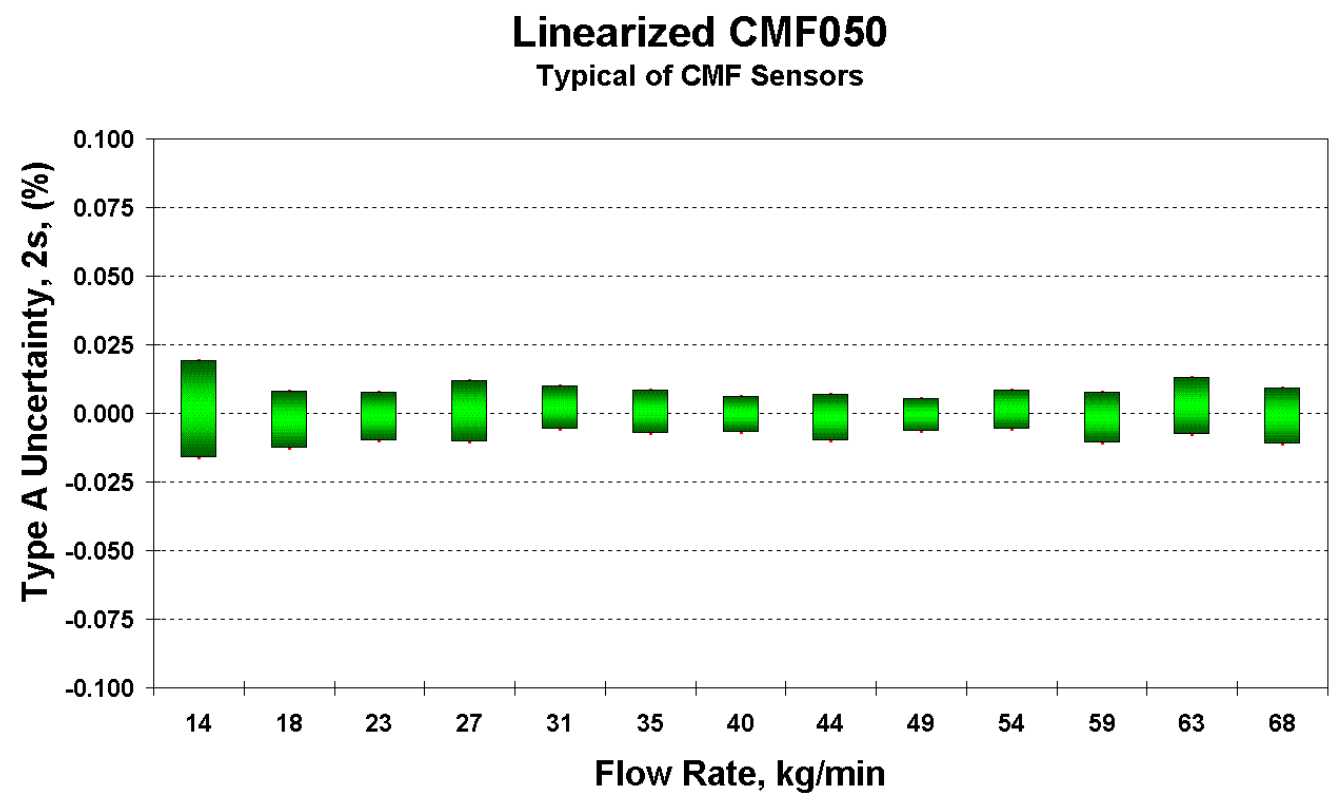

Figure 1. Typical TSM RM type A Uncertainty

\section{TSM OPERATION}

Figure 2 is a simplified P\&ID (process \& instrument diagram) of the TSM design. Different TSM calibration stands with different flow rate capabilities are all of a similar design. All TSM stands use pneumatically powered clamping systems, which facilitate rapid installation of the UUT (unit under test refers to the meter(s) being calibrated and adjusted). The flow meters called RM (reference meters) and QCM (quality check meter) on each TSM stand are identical in size and calibration. For some meter technologies, the UUT can be more than one sensor installed in series.

The UUT is calibrated and adjusted to the RM. When verifying a meter's performance, after determining its flow calibration factor, the TSM calibration stand with its automated programming checks the UUT's low flow performance by comparing it to that of one of the inline reference meters smaller than itself. Each time a reading is obtained from the UUT, the RM and QCM meters are also read. This is one of the key features of the TSM stand design: The QCM provides a continuous, real time, first level check on the calibration process for the UUT and on the stability of the RM, as well as on other potentially confounding effects such as entrained air and leakage. 


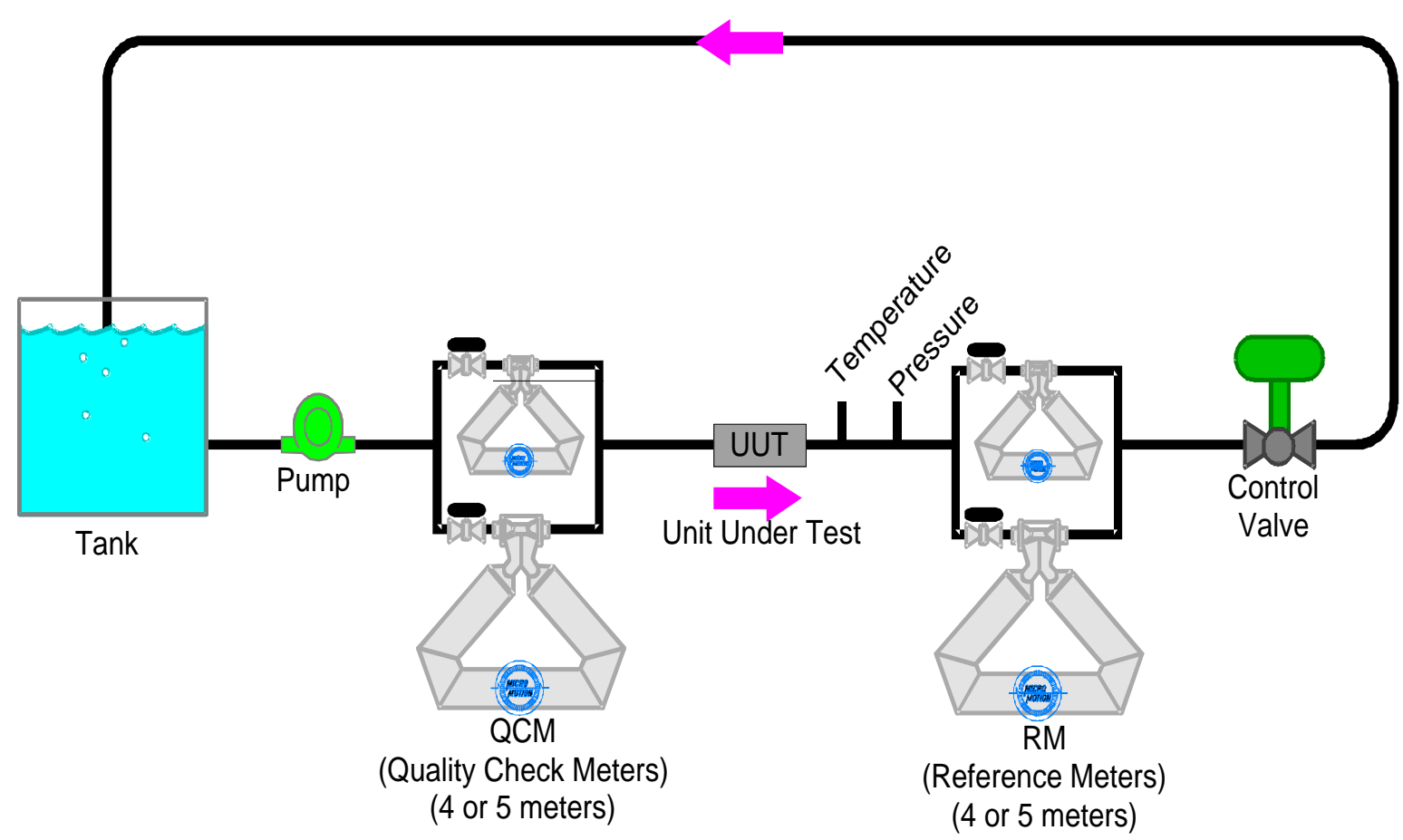

Figure 2. Simplified P \&ID for a TSM Flowmeter Calibration Stand

\section{TRACEABILITY}

\subsection{Calibration Traceability for TSM Stands}

The uncertainty of the TSM calibration process is traced to national standards through a chain of successive measurements (figure 3 ). The USA traceable mass standards are used for calibration of the MMI Primary Stand scales. (The words, box 1, box 2, box 3 and box 4 shown on the traceability diagram, figure 3 , are not a part of the traceability chain but are there as a convenience to use for reference in other parts of this paper.) NMI - VSL (Physical Standards Laboratory of the Netherlands) has approved this method for generating traceability for the MMI TSM stands (reference 1).

The RM meters (figure 2) are the reference standards in the TSM calibration stand, and they are calibrated on the MMI Primary Stand (figure 3, box 2). The MMI Primary Stand uses three high-precision scales for the calibration of the RM and QCM meters, in order to cover the full range of required flow rates.

All links in the traceability chain are renewed periodically. All TSM meters are returned to the MMI Primary Stand on a regular basis for verification. The scales on the MMI Primary Stand are validated with the MMI mass standards. The MMI working mass standards traceable to NIST (National Institute of Science and Technology) are returned to the State of Colorado for calibration every year.

The calibration of all temperature, pressure and barometric equipment for all MMI flowmeter stands are verified on a regular basis with standards traceable to NIST. 


\subsection{Multi-path Traceable Verification for TSM Process}

Two sets of reference mass standards are maintained on site for verification of the three scales of the MMI Primary Stand (figure 4). One set of mass standards is traceable to USA national standards and the other set is traceable to Japanese national standards. The scale calibrations with their correction curves are verified using both the USA-traceable and the Japantraceable mass standards.

Both sets of the reference masses follow similar paths through third parties to be traceable to their respective national standards. The NIST traceable mass standards are calibrated by the State of Colorado. The NRLM (National Research Laboratory of Metrology) traceable mass standards are calibrated by JQA (Japan Quality Assurance Institute). The State of Colorado and JQA perform the calibration of the MMI mass standards by comparison to their working standards, which in turn are traceable to their resident primary standards, which are then traceable to the respective national standards

\section{MEASUREMENT ASSURANCE}

As stated earlier, the first level of measurement assurance is provided by the QCM meters, which are an integral part of each TSM stand. The automated checking of the RM flow measurement against the QCM flow measurement that occurs for each data point provides an online, continuous verification of the RM by an equivalent check standard.

A second level of measurement assurance utilizes so-called "gold" meters. These gold meters (which are also standard Micro Motion Elite meters) constitute another type of check standard, and a set of them are permanently located and maintained at each MMI facility with TSM stands and in the laboratory where the MMI Primary Stand is located. The gold meters provide measurement assurance for the overall TSM process by regularly verifying them in the UUT sections of the TSM stands and the MMI Primary Stand. These gold meters are always reverified without making adjustments to them, so that the data collected serves as a measure of the variation over time of the TSM or MMI Primary Stand. The gold meters used for the MMI Primary Stand are also used in the same capacity on several other flow facilities that reside in the MMI Engineering laboratory, thus providing an ILC (inter-laboratory comparison).

A third method for measurement assurance is a broader ILC that includes several TSM and gravimetric flowmeter calibration stands. In this case, the scales of the gravimetric stands are calibrated using a different set of NIST traceable working standards, not the same ones used to calibrate the scales for the MMI Primary Stand. An even broader ILC, which would include multiple TSM stands and some national flow standards laboratories, is being planned. 


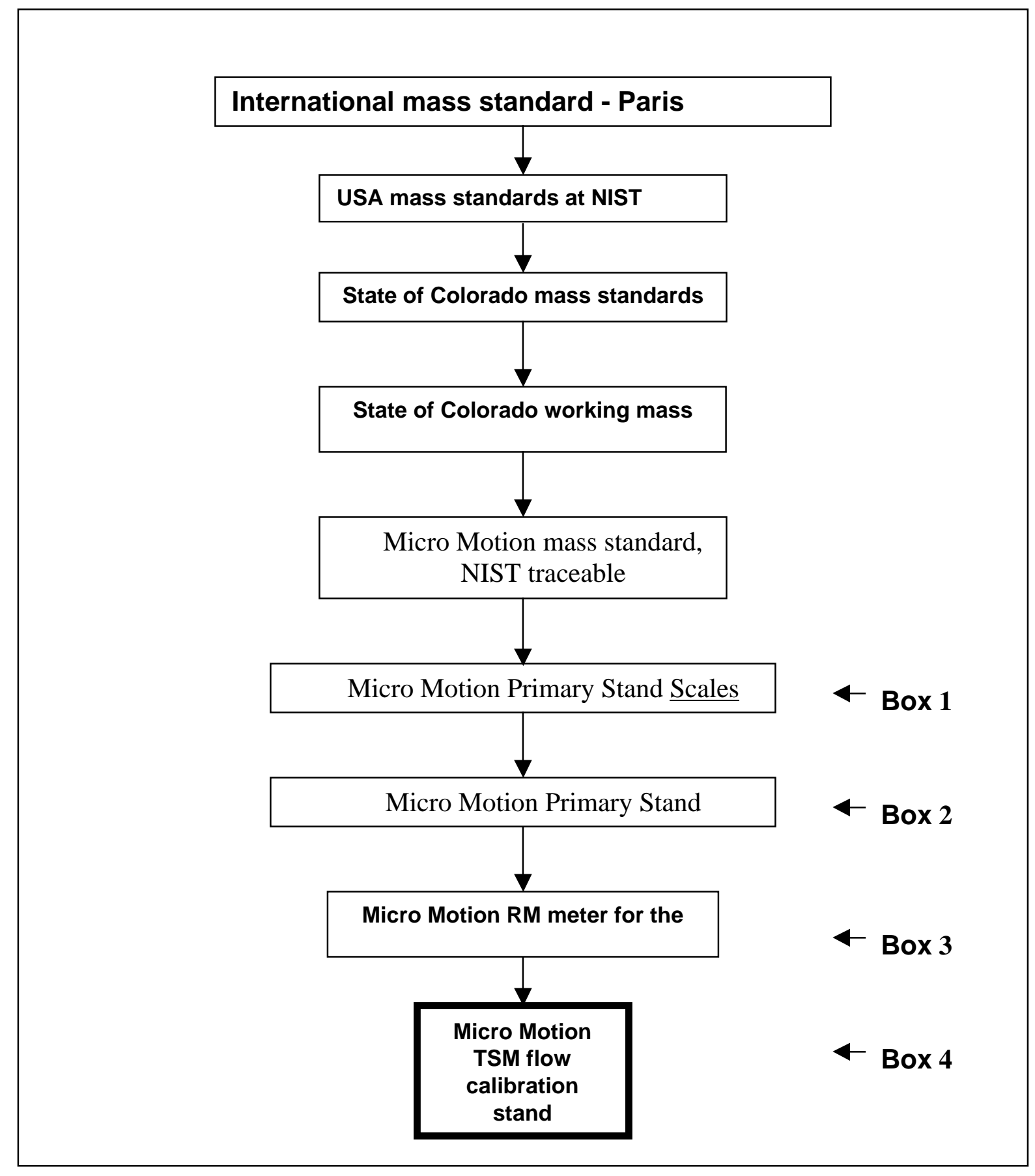

Figure 3. Calibration Traceability for TSM Stands 


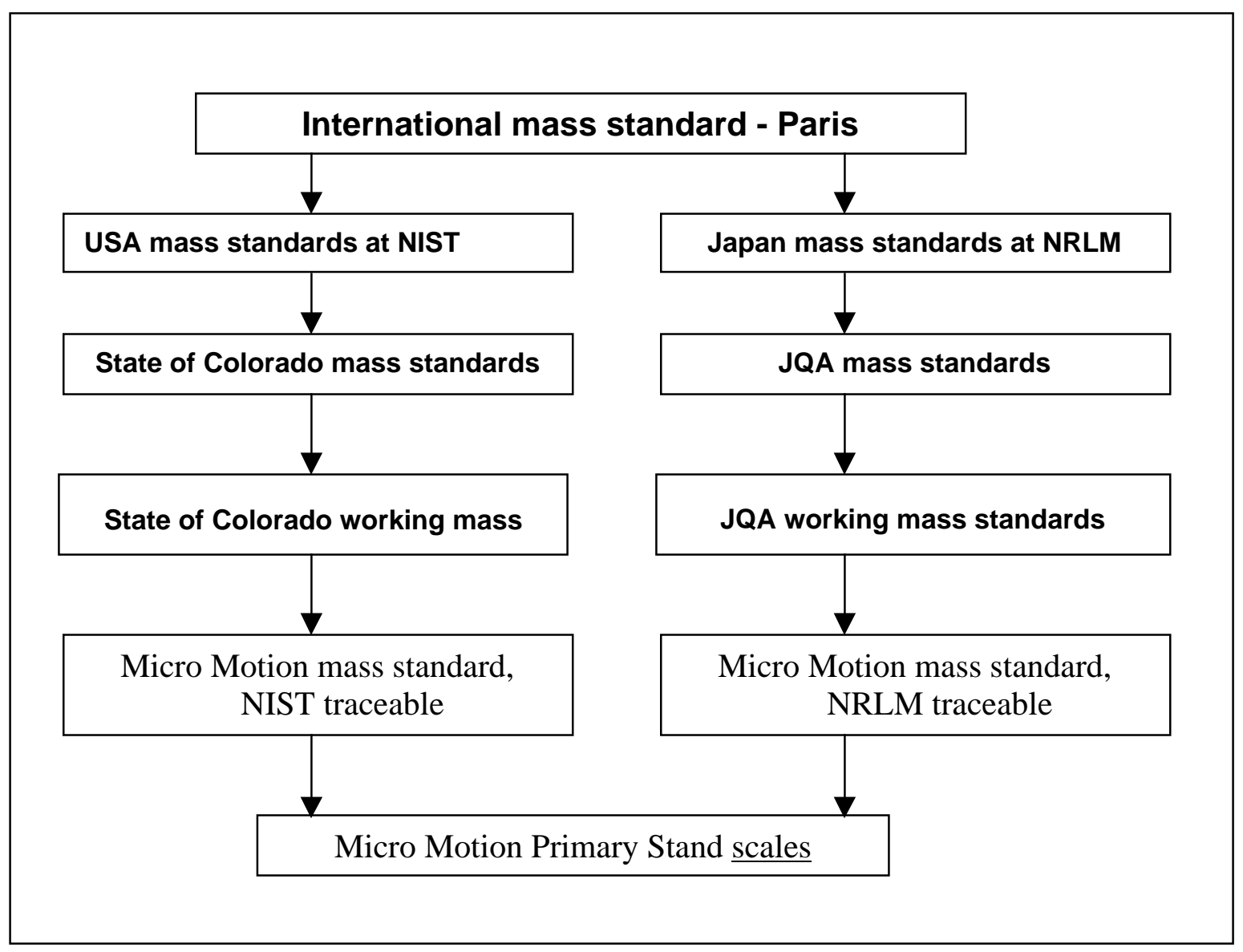

Figure 4. Multi-path Traceable Verification for TSM Process

\section{UNCERTAINTY ANALYSIS}

\subsection{General}

Definitions:

- The word uncertainty in this paper means "expanded uncertainty" (> 95\% confidence).

- BMC (Best Measurement Capability) refers to the lowest uncertainty of measurement at which a flow meter calibration stand can operate, in the hypothetical situation that the UUT in the stand is perfect (i.e., the UUT's type A uncertainty is zero).

\section{This analysis is guided by:}

- GUM (Guide to the Expression of the Uncertainty in Measurement). The total uncertainty for any application is calculated as the root of the sum of the squares (RSS) combination of each of the individual contributions to that application's uncertainty. Reference 2.

- Uncertainty Analysis of stand 4 at MMI/Veenendaal, NL, as performed by NMi-VSL. Reference 3.

- Calculation of Liquid-mass (in vacuo) out of Weighing Scale Indication and its Related Uncertainty. Reference 4.

The uncertainty analysis for the Micro Motion TSM flow calibration stand consists of four parts: 
a) Uncertainty of the scale - part of the MMI Primary Stand (Figure 3, Box 1)

b) Uncertainty of the MMI Primary Stand (BMC, mass, Figure 3, Box 2)

c) Uncertainty of the reference meter (Figure 3, Box 3)

d) Uncertainty of the TSM flowmeter calibration stand (BMC, mass and volume, Fig. 3, Box 4)

7.2 Uncertainty of the scales, in the mean, of the MMI Primary Stand (Figure 3, Box 1)

The three high-precision scales used in the MMI Primary Stand have been optimized for best performance in the following ways:

- The liquid container is centrally located on the weighing scale platform so the water weight is uniformly spread with no eccentric loads.

- The scales are only used with increasing loads to avoid hysteresis effects.

- The scales are installed indoors so ambient temperature changes are minimal.

- When the container is drained, it is always drained completely.

In addition the MMI Primary Stand incorporates the following capabilities:

- Online air density determination during scale and RM calibration.

- Online water density determination during RM calibration.

All mass standards used have known density and density uncertainty, as specified on their calibration certificates.

These subjects are considered when determining the uncertainty of the scale, and since the scales are used in the traceability chain, "uncertainty in mean" is calculated.

\subsubsection{Type A Uncertainty in the Mean of the Scales (Figure 3, Box 1)}

An appropriate minimum batch size was selected for each of the three scales in the MMI Primary Stand (2, 200, and $1500 \mathrm{lbs}$, respectively, for the small, medium, and large scales on this stand). Repetitive measurements were made at these minimum batch sizes across the full range of each scale in accordance with section 7.2. These data were then analyzed to establish the type A uncertainty in the mean for each scale:

$U_{A M-S C A L E S}=2 s / \sqrt{n}$

$=$ The type A uncertainty in the mean for each scale. (Equal to the type A uncertainty in the single measurement (repeatability) divided by the square root of $\mathrm{n}$, the number of measurements.) Typically, a hundred or more individual measurements were used in the calibration process for each scale.

Table 1. Type A uncertainty in the mean of the scales

\begin{tabular}{|l|l|l|l|}
\hline & Small Scale & Medium Scale & Large Scale \\
\hline Minimum Batch (lb) & $\mathbf{2}$ & $\mathbf{2 0 0}$ & $\mathbf{1 5 0 0}$ \\
$U_{A M-S C A L E S}$ & $\mathbf{0 . 0 0 5 2} \%$ & $\mathbf{0 . 0 0 3 3 \%}$ & $\mathbf{0 . 0 0 8 0} \%$ \\
\hline
\end{tabular}




\subsubsection{Type B Uncertainty of the Scales (Figure 3, Box 1)}

Type B uncertainty is frequently referred to as systematic uncertainty or bias.

The mass is calculated from the scale indication during the scale calibration as follows:

Mass = scale-indication $\mathrm{x}$ WSF

$\mathrm{WSF}=$ Weigh Scale Factor $($ reference 4$)=0.999876$ (average WSF during scale calibration)

Four sources of type B uncertainty influence the mass indicated by the scales:

a) $U_{B 1}=$ Uncertainty of the mass standards $(0.002 \%$, from certificates).

b) $U_{B 2}=$ Uncertainty related to the resolution of the scale readout and the scale sensitivity. The controlling factor between these two items is the scale readout, and the uncertainty of it is determined using a rectangular distribution $(0.0058 \%$ to $0.0077 \%)$. Reference 4 .

c) $U_{B 3}=$ Uncertainty of the air density during scale calibration, due to the equipment used and varying ambient conditions (1\%, confidence level > 95\%).

d) $U_{B 4}=$ Uncertainty of the density of the mass standards used for scale calibration (5\%).

Table 2. Type B uncertainty of the scales

\begin{tabular}{|c|c|c|c|c|}
\hline Uncertainty Source & $\begin{array}{l}\text { Calculation } \\
\text { Method }\end{array}$ & $\begin{array}{l}\text { Small Scale } \\
\text { (Percent) }\end{array}$ & $\begin{array}{l}\text { Medium Scale } \\
\text { (Percent) }\end{array}$ & $\begin{array}{l}\text { Large Scale } \\
\text { (Percent) }\end{array}$ \\
\hline Mass Standards & $U_{B 1} S 1$ & $0.002 \times 1$ & $0.002 \times 1$ & $0.002 \times 1$ \\
\hline Scale Readout & $U_{B 2} S 2$ & $0.0058 \times 1$ & $0.0058 \times 1$ & $0.0077 \times 1$ \\
\hline Air Density & $U_{B 3} S 3$ & $1.0 \times 0.00012$ & $1.0 \times 0.00012$ & $1.0 \times 0.00012$ \\
\hline $\begin{array}{l}\text { Mass Standard } \\
\text { Density }\end{array}$ & $U_{B 4} \mathrm{~S} 4$ & $5.0 \times 0.00003$ & $5.0 \times 0.00003$ & $5.0 \times 0.00003$ \\
\hline$U_{B T-S C A L E S}$ & & 0.0061 & 0.0061 & 0.0080 \\
\hline
\end{tabular}

Notes

a) $U_{B 2}$ is for minimum batch quantity.

b) $S 1$ to $S 4$ are sensitivity coefficients, the influence factors of the uncertainty source on the scale indications (reference 4).

7.2.3 Combined Uncertainty in the Mean for the Scales (Figure 3, Box 1) 
This uncertainty value, $U_{C M-S C A L E}$, is calculated as the RSS combination of type A and type B uncertainties of the scales, per sections 7.2.1 and 7.2.2 above.

Table 3. Combined uncertainty in the mean for the scales

\begin{tabular}{|l|l|l|l|}
\hline & $\begin{array}{l}\text { Small Scale } \\
\text { (Percent) }\end{array}$ & $\begin{array}{l}\text { Medium Scale } \\
\text { (Percent) }\end{array}$ & $\begin{array}{l}\text { Large Scale } \\
\text { (Percent) }\end{array}$ \\
\hline$U_{A M-S C A L E}$ & $\mathbf{0 . 0 0 5 2}$ & $\mathbf{0 . 0 0 3 3}$ & $\mathbf{0 . 0 0 8 0}$ \\
$U_{B T-S C A L E}$ & $\mathbf{0 . 0 0 6 1}$ & $\mathbf{0 . 0 0 6 1}$ & $\mathbf{0 . 0 0 8 0}$ \\
$U_{C M-S C A L E}$ & $\mathbf{0 . 0 0 8 0}$ & $\mathbf{0 . 0 0 6 9}$ & $\mathbf{0 . 0 1 1 3}$ \\
\hline
\end{tabular}

\subsection{BMC of MMI Primary Stand (Figure 3, Box 2)}

The mass of the liquid, measured with the TSM RM meter, is calculated as follows:

Mass $=$ Scale indication $\mathrm{x}$ WSF $\mathrm{x}$ BVC

$\mathrm{WSF}=$ Weigh Scale Factor (See section 7.2.2)

$\mathrm{BVC}=$ Buoyancy Vapor Correction (reference 4) $=1.000994$ (average BVC during RM calibration)

The BMC for mass of the MMI Primary Stand (BMC $\left.{ }_{\text {PRIMARY STAND-MASS }}\right)$ is influenced by five parameters, and these are again combined in an RSS fashion:

a) $U_{5}=$ the combined uncertainty in the mean of the scale $(0.0069$ to $0.0113 \%, S 5=1)$.

b) $U_{6}=$ uncertainty related to air density during operation $(1.0 \%, S 6=0.001)$.

c) $U_{7}=$ uncertainty related to liquid density during operation. The uncertainty of the liquid density is $0.015 \%$ in section 7.6. This value is for the density of the water in the pipe near the UUT. What is needed is the density of the water in the tank. The density uncertainty was increased to allow for $1 / 2$ degree $\mathrm{C}$ temperature difference between the pipe and scale $\quad \operatorname{tanks}(0.020 \%, S 7=0.001)$.

d) $U_{8}=$ uncertainty, caused by the "transfer point", based on a maximum of $1 \mathrm{~mm}$ variation from the mean and a rectangular distribution $(0.0088 \%$ to $0.0015 \%, S 8=1)$. Reference 3 .

e) $U_{9}=$ uncertainty related to the minimum number of pulses of $\operatorname{RM}(0.0025 \%, S 9=1)$. 
Table 4. BMC of MMI Primary Stand

\begin{tabular}{|l|l|l|l|l|}
\hline Uncertainty Source & $\begin{array}{l}\text { Calculation } \\
\text { Method }\end{array}$ & $\begin{array}{l}\text { Small Scale } \\
\text { (Percent) }\end{array}$ & $\begin{array}{l}\text { Medium Scale } \\
\text { (Percent) }\end{array}$ & $\begin{array}{l}\text { Large Scale } \\
\text { (Percent) }\end{array}$ \\
\hline Scales & $U_{5} S 5$ & $0.0080 \times 1$ & $0.0069 \times 1$ & $0.0113 \times 1$ \\
Air Density & $U_{6} S 6$ & $1 \times 0.001$ & $1 \times 0.001$ & $1 \times 0.001$ \\
Liquid Density & $U_{7} S 7$ & $0.020 \times 0.001$ & $0.020 \times 0.001$ & $0.020 \times 0.001$ \\
Transfer Point & $U_{8} S 8$ & $0.0088 \times 1$ & $0.0037 \times 1$ & $0.0015 \times 1$ \\
Pulses & $U_{9} S 9$ & $0.0025 \times 1$ & $0.0025 \times 1$ & $0.0025 \times 1$ \\
BMC $_{\text {PRMARY STAND-MASS }}$ & & 0.0122 & 0.0083 & 0.0117 \\
\hline
\end{tabular}

Notes: $S 5$ to $S 9$ are sensitivity coefficients, the influence factors of the uncertainty source on the ${ }_{B M C_{\text {PRMARY STAND-MASS }}}$ (reference 4$)$

7.4 Uncertainty of TSM Reference Meters, Calibrated on MMI Primary Stand (Figure 3, Box 3)

BMC in mass of the MMI Primary Stand is now known, per the previous section. Next, the uncertainty of the RMs that are calibrated on this stand $\left(U_{C M-R M}\right)$ is determined by the RSS combination of this value with the type A uncertainty of the TSM RMs themselves. Figure 1 shows an example of the data used for this analysis.

From figure 1 the type A uncertainty of the RMs $\left(U_{A M-R M}\right)$ is less than $\left(\frac{0.020}{\sqrt{25}}\right)=0.0040$ $\%$, since 25 measurements were made at each individual flow rate.

Then $U_{C M-R M}=\sqrt{U_{A M-R M}^{2}+B M C_{\text {PRIMARY STAND-MASS }}^{2}}$

Since the $B M C_{\text {PRIMARY STAND-MASS }}$ is different for each scale, there could be a TSM RM uncertainty dependent on scale used. This paper will only calculate the worst case, as follows.

$$
U_{C M-R M}=\sqrt{(0.0040)^{2}+(0.0122)^{2}}=0.0128 \%
$$

7.5 BMC of TSM Stand, Mass (Figure 3, Box 4)

When an RM is installed in the TSM stand, then its uncertainty is part of the calculation of the Best Measurement Capability of the TSM stand. The TSM method is based on steady state flow, no uncertainty is introduced due to the transfer point because there is no transfer point. However, there is instead a different component to the BMC of the TSM stand due to the number of pulses counted during each measurement, and the uncertainty associated with this process.

Therefore the BMC in mass of the TSM stand $\left(B M C_{T S M-M A S S}\right)$ is determined from the combined RSS uncertainty of the RM meter $\left(U_{C M-R M}\right)$, per section 7.4 above, and the uncertainty related to the number of pulses used. The minimum number of pulses used in all TSM stand measurements is 50,000. Hence, we get 


$$
B M C_{T S M-M A S S}=\sqrt{U_{C M-R M}^{2}+U_{\text {Pulses }}^{2}}=\sqrt{(0.0128)^{2}+(0.0020)^{2}}=0.013 \%
$$

\subsection{BMC of TSM Stand, Volume (Figure 3, Box 4)}

The BMC of the TSM stand in volume $\left(B M C_{T S M-V O L U M E}\right)$ can be calculated from $B M C_{T S M-M A S S}$ and uncertainty of the water density, $U_{\rho T \text { density }}$. Measurements on the system have shown the bias between the system water supply and NIST samples to be constant. The water density uncertainty analysis includes an Anton-Paar densitometer, a certified water sample from NIST with its uncertainty analysis, samples from of the TSM system water, water temperature measurements, and water pressure measurement and sampling factors. All together, the uncertainty analysis for water density includes eight items. These eight items and their uncertainties are modeled after work completed by NMi on a gravimetric stand at MMI, Veenendaal, NL (reference 3). These eight influences, as before, are combined in an RSS fashion to determine the uncertainty of the density determination, $U_{\rho T \text { density }}$. This information is summarized in Table 5.

Table 5. Uncertainty Analysis for Water Density

\begin{tabular}{|l|l|}
\hline & \multicolumn{1}{|c|}{$\begin{array}{c}\text { Contribution to } \\
\text { Uncertainty } \\
\text { (Percent) }\end{array}$} \\
\hline Uncertainty of the sample from the national laboratory used to calibrate the Anton-Paar & 0.00100 \\
Resolution uncertainty of the Anton-Paar on national laboratory sample & 0.01000 \\
Resolution uncertainty of the Anton-Paar on TSM system water sample & 0.01000 \\
Basic uncertainty of the PTB equations for density of water & 0.00050 \\
Uncertainty of the calibration of the temperature device & 0.00420 \\
Uncertainty due the sampling techniques of the temperature & 0.00210 \\
Uncertainty of the calibration of the pressure device & 0.00046 \\
Uncertainty due to the sampling techniques of the pressure & 0.00046 \\
\hline & \\
\hline
\end{tabular}

Finally, $U_{\rho T \text { density }}$ is combined with $B M C_{T S M-M A S S}$ from section 7.5 to calculate the BMC of the TSM stand on volume, $B M C_{T S M-V O L U M E}$, as follows:

$$
B M C_{T S M-V O L U M E}=\sqrt{B M C_{T S M \text { Mass }}^{2}+U_{\rho T \text { density }}^{2}}=\sqrt{(0.0131)^{2}+(0.0150)^{2}}=\mathbf{0 . 0 2 0} \%
$$

\section{CONCLUSION}

The end result of this analysis, from the previous two sections of this paper, is a Best Measurement Capability of the TSM system of $0.013 \%$ on mass and $0.020 \%$ on volume. These values, even for the worst case conditions they represent, show that the BMC's of the TSM stands well exceed the target performance of $0.033 \%$ for calibration stand capability established in section 2 of this paper. Micro Motion has applied to the Dutch Accreditation Council RvA 
(RAAD VOOR ACCREDITATIE) in March 2000, for accreditation of the MMI Primary Stand. The accreditation process is continuing at this time.

\section{REFERENCES}

1. Oral communications with Mr. J.G.M. van der Grinten/NMi-VSL

2. GUM - Guide to the Expression of Uncertainty in Measurement/ISO/OIML/IEC

3. Unpublished - Uncertainty Analysis of stand 4 at MMI/FisherRosemont/Veenendaal, NL/by Mr. J.G.M. van der Grinten/NMi-VSL

4. Unpublished - Calculation of Liquid-mass (in vacuo) out of Weighing Scale Indication and its Related Uncertainty/ by Aart Pruysen/Fisher Rosemount

AUTHORS: Robert DeBoom (303.530.8517), Marc Butler (303.530.8562), Andrew Kolbeck (303.530.8552), Micro Motion, Inc, Boulder CO, 80301, Aart Pruysen (31.3185.49564), Fisher-Rosemount, Veenendaal, NL.E-mail: bobd@micromotion.com, marcb@micromotion.com, andyk@micromotion.com, aart.pruysen@frco.com 


\section{Appendix C}

Test Report 


\title{
Appendix C
}

\section{Test Report}

\author{
June 26, 2000 \\ DS150S, S/N 138085 \\ Enron / Northern Natural Gas
}

\section{Introduction}

The Redfield, Iowa facility is an underground storage facility for natural gas. Pipeline gas is "pumped" into the storage facility (really a big aquifer in a porous sandstone / shale formation) during the off-peak summer months, then removed beginning in November. The facility has operated since the mid-1950's, using primarily bi-directional orifice plates for accounting purposes at approximately 70 points around the field. Alternative technologies have been investigated over the years, including Coriolis meters.

The labor to properly maintain and calibrate the orifice plates was the primary motivation for looking for a different technology. A major component of the labor requirements was the need to reconfigure the meters for changing flow rate. The pressure of the facility decreases throughout the year as gas is drawn out, and the flow rate drops accordingly. To accommodate the changing pressure and rate, the pressure and delta-pressure transmitters must be re-spanned and the beta-ratio of the orifice must be changed, at each of the 70 measurement points. Additionally, the gas when it is removed is dirty and sour, causing impulse lines plug plugging problems and orifice plate maintenance issues.

It was hoped that the Coriolis meter would address the orifice plate issues and maintenance costs by improving:

$>$ High turndown capabilities of the Coriolis meter would eliminate the need for any re-ranging

$>$ Maintenance and calibration costs associated with coating and plugging would be less

$>$ Maintaining bi-directional usage

\section{Coriolis Meter Installation and Performance}

The Coriolis meter (DS150S) was installed as shown below. 


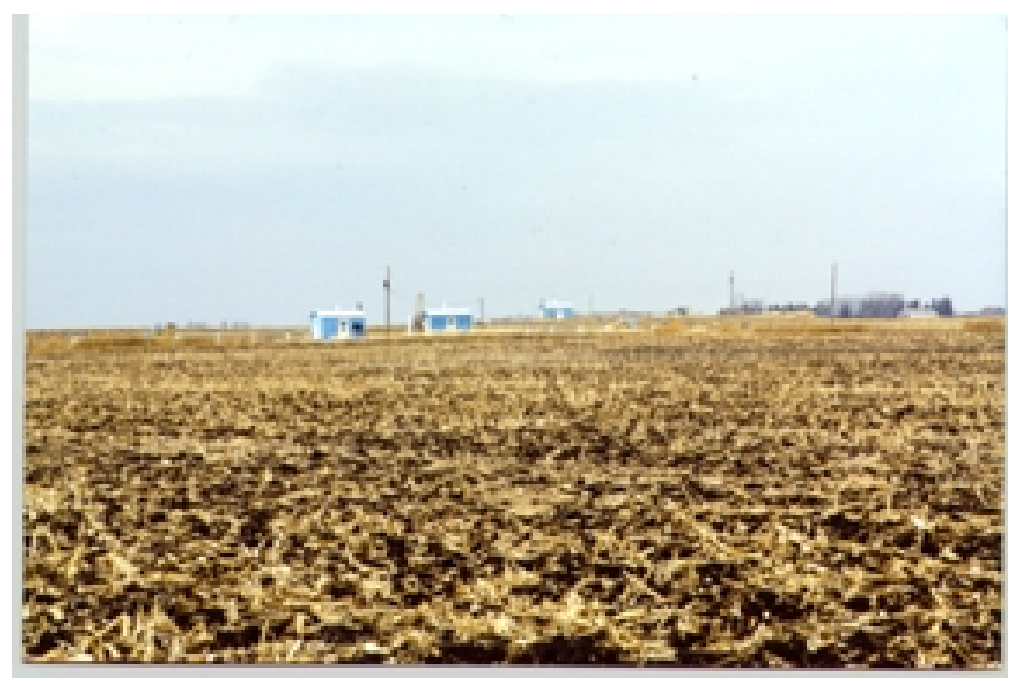

Figure 1. Metering Stations are housed in the blue "sheds" at approximately 70 locations

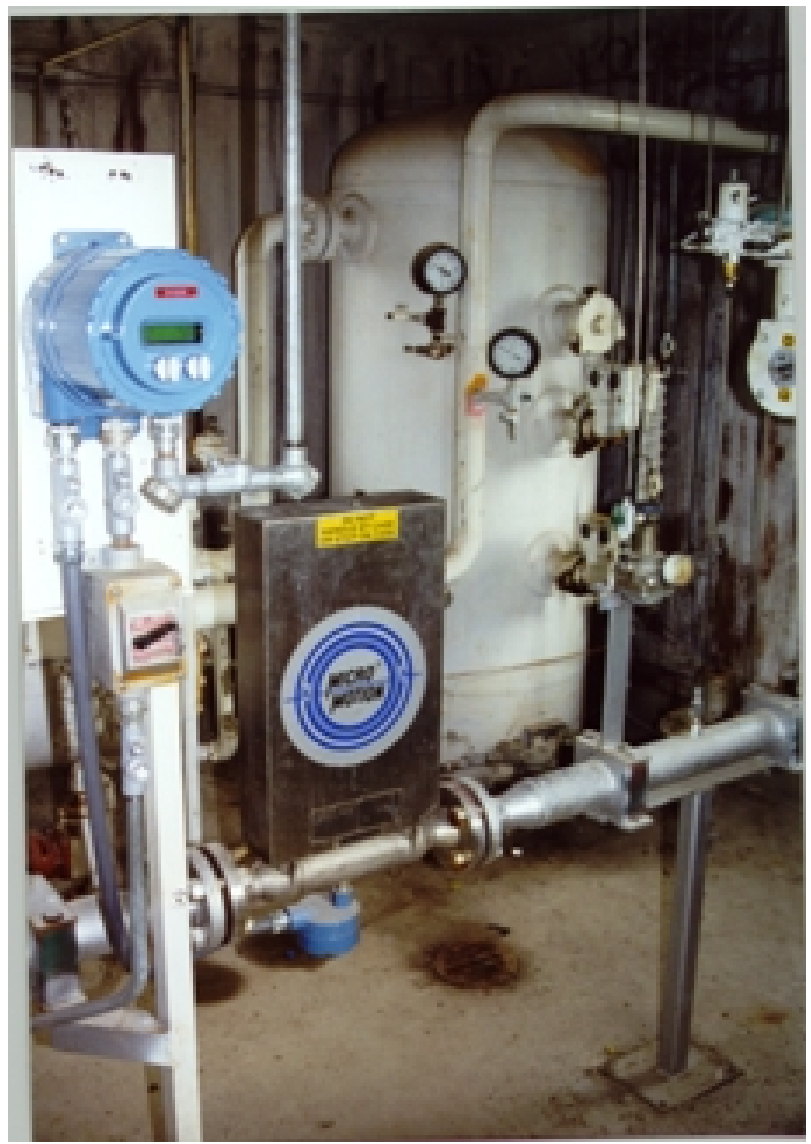

Figure 2. DS150S installed in one of the blue "sheds" 


\section{Meter Performance}

Enron has always thought that the DS150S was performing well, but the meter had not been proved or calibrated since installation in 1991. In May, 2000 the meter was removed during a plant turn-around and returned to Micro Motion for a check of the calibration. Using the calibration constants from the sensor tag (established in 1991), a standard water check as well as a gas calibration on compressed air were performed.

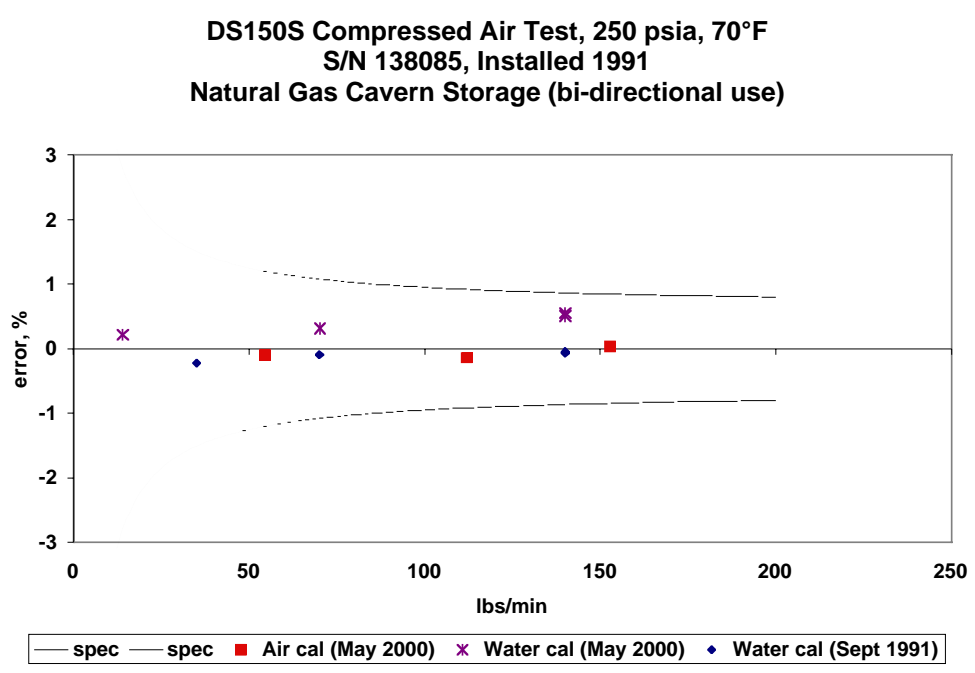

Figure 3. DS150S Calibration check results

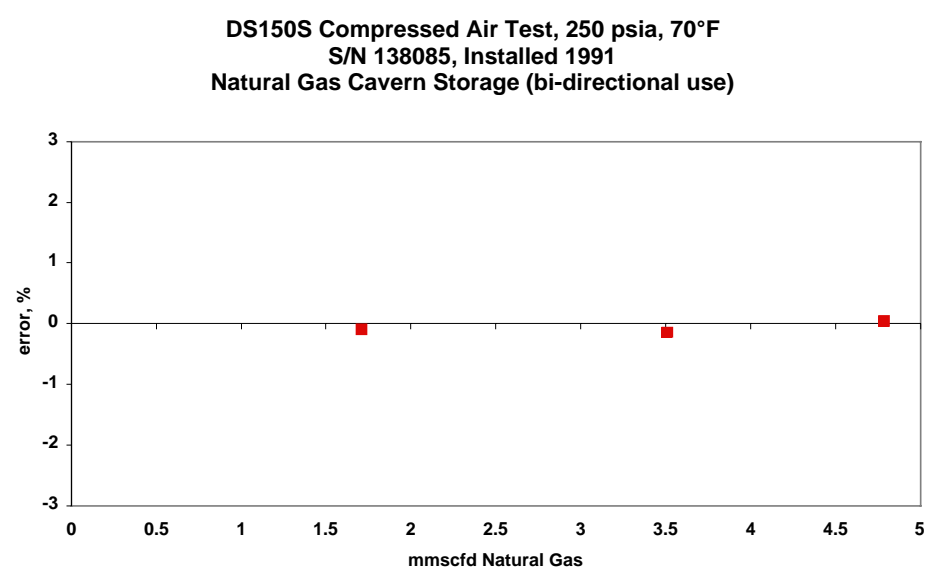

Figure 4. Air calibration data shown in natural gas units

\section{Conclusions}

The meter has exceeded the customer's expectations for reliability and stability. In 9 years of operation, no maintenance on the meter was performed indicating that the plugging / coating problems of the orifice plates did not happen with the DS150S meter. During the same period, the perceived accuracy has always been good, and now there is data to confirm that performance is well within specification, with accuracy better than $+/-0.5 \%$. 


\section{Appendix D}

Informal Collection of Users of Micro Motion Meters in Radioactive Environments and/or Nuclear Applications 
$6 / 12 / 02$

"Informal" Collection of Micro Motion Meters in Radioactive Environments and/or Nuclear Applications

Palo Verde Nuclear Power Plant-Arizona: Boronated Water

(Richard Persohn)

University of Rochester-New York: Laser Energetic Lab

(Darren Whitford)

INEEL-Idaho: Various meters on radioactive waste and processes

(Craig Powell, Phillip Beasley)

Turkey Point Nuclear-Florida: Water

(Ray Ecklebarger)

Rocky Flats-Colorado: Uranium slurry

(Tom Argue)

Rocky Flats-Colorado: Weapons decommissioning

(Marion Ignaffo)

Los Alamos Labs-New Mexico: Specific process unknown

(Brent Pankonian)

Savanna River-Georgia: Specific process unknown

(Bob Adams)

GE Nuclear Fuels-North Carolina: Uranium Hexaflouride

(Kevin Wiseman)

Nuclear Waste Cleanup-Oakridge, Tennessee: process unknown

(Joe Cipriani)

Oakridge National Labs-Tennessee: 40-50 meters

X-10 Oakridge National Labs

Y-12 Nuclear Weapons Facility

K-25 Gaseous Diffusion Process Facility

(Steve McCall)

Honeywell (previously Allied Signal)-Metropolis, Illinois: Uranium Hexaflouride (Troy Mclean) 


\section{Appendix E}

\section{Materials in CMF 100 and T 100 Micro Motion Meters}




\section{Appendix E \\ Materials in CMF 100 and T 100 Micro Motion Meters}

\begin{tabular}{||l|l|}
\hline \multicolumn{1}{|c|}{ CMF100 Materials } & \multicolumn{1}{|c|}{ T100 Materials } \\
\hline Metals & Metals \\
\hline Stainless steel including, but not limited to & Stainless steel including, but not limited to 304L, \\
316L, 304L, 308L, carbon steel, CF-3M & Silver plated alloy wires \\
\hline Silver plated alloy wires & Copper \\
\hline Copper & Platinum \\
\hline Monel & Brass \\
\hline 52 Alloy & ER312 \\
\hline Platinum & Titanium \\
\hline & CDA Alloy \#110 \\
\hline Adhesives & \\
\hline Silicone & Adhesives \\
\hline Fiberglass tape & Silicone \\
\hline Loctite 272 & Loctite 272 \\
\hline T-Gon 340 & T-Gon 340 \\
\hline Acrylic & \\
\hline & Coatings \\
\hline Coatings & Silicone \\
\hline Silicone & Fiberglass sleeving \\
\hline Fiberglass sleeving & Teflon \\
\hline PTFE & Polymide \\
\hline Polymide & Dow 1-2577 Conformal Coating \\
\hline Dow 1-2577 Conformal Coating & Thermoplastic \\
\hline Dow 3-1744 Conformal Coating & Polyvinylidene Fluoride heat shrink sleeving \\
\hline & \\
\hline Miscellaneous & Miscellaneous \\
\hline Boron Nickel-2 filler & Boron Nickel-2 filler \\
\hline Silver and Tin Solder & Silver and Tin Solder \\
\hline SM1-CO5 Grade 18 & SM1-CO5 Grade 18 \\
\hline Rosin Flux 800 & Rosin Flux 800 \\
\hline Polyphenylen Sulfide with 40\% glass & Polyphenylen Sulfide with 40\% glass \\
\hline Glass & Ceramic \\
\hline Ceramic & Silver Braze \\
\hline Metalized Polyester Film & \\
\hline & \\
\hline
\end{tabular}


PNNL-13485

Rev. 1

\section{Distribution}

No. of

Copies

Offsite

M. Katona

Department of Civil and Environmental Engineering

Washington State University

Pullman, WA 99164

\section{Onsite}

2 DOE Richland Operations Office

E. J. Cruz

H6-60

J. J. Davis

H6-60

21 CH2MHILL Hanford Group, Inc.
B. J. Eischens
R3-47
A. H. Friberg
R3-83
T. G. Goetz
R1-49
J. M. Grigsby
R1-44
G. A. Hanson
H. R. Hopkins II
H6-16
S7-83
N. W. Kirch
M. A. Knight
R3-73
R2-11
R2-39
J. G. Kristofzski
R3-47
E. W. Martinen
G5-58
C. H. Mulkey
R1-51
S. M. O'Toole
S7-65
D. L. Parker
R2-37

No. of

Copies
R. P. Raven
S7-02
D. A. Reynolds
R2-11
K. S. Tollefson
R1-51
J. E. Van Beek
R3-47
J. A. Voogd
R2-50

1 Fluor Daniel Hanford Company

D. L. Herting

T6-07

9 NUMATEC Hanford Company
R. R. Bafus
S5-05
S. R. Briggs
R3-47
P. J. Certa
L4-07
T. Choho
R3-25
T. J. Conrads
R3-83
C. E. Grenard (3)
R3-83
C. A. Rieck
S0-11

28 Pacific Northwest National Laboratory

S. Q. Bennett

K7-90

J. W. Brothers

K9-20

R. A. Brouns

K9-01

J. L. Buelt

K9-09

C. W. Enderlin (5)

$\mathrm{K} 7-15$

P. A. Gauglitz

K6-28

J. L. Huckaby

$\mathrm{K} 7-15$

W. L. Kuhn

K7-15

Y. Onishi (10)

K7-15

C. W. Stewart

$\mathrm{K} 7-15$

B. E. Wells (3)

K7-15

Information Release (2) 